\title{
Zinc Oxide Nanoparticles Synthesis Methods and its Effect on Morphology: A Review
}

\author{
Eric Kwabena Droepenu ${ }^{1,2, * \mathbb{C}}$, Boon Siong Wee ${ }^{1, * \mathbb{C}}$, Suk Fun Chin ${ }^{1} \mathbb{D}$, Kuan Ying Kok ${ }^{3 \mathbb{C}}$, \\ Muhammad Firdaus Maligan 1(i) \\ 1 Resource Chemistry Program, Faculty of Resource Science and Technology, Universiti Malaysia Sarawak 94300, Kota \\ Samarahan, Sarawak, Malaysia \\ 2 Graduate School of Nuclear and Allied Sciences, University of Ghana, AE1, Kwabenya-Accra, Ghana \\ 3 Malaysian Nuclear Agency, Bangi, Kajang, 43000 Selangor, Malaysia \\ * Correspondence: swboon@ unimas.my (B.S.W.); kobladodzie01@ yahoo.com (E.K.D.);
}

Scopus Author ID 57194506096

Received: 28.06.2021; Revised: 1.08.2021; Accepted: 5.08.2021; Published: 14.08.2021

\begin{abstract}
Zinc oxide is an important material with numerous applications due to its unique properties. Due to their thermal and chemical stability are used in wide applications such as LEDs, sensors, catalysts, and photodetectors. Different chemical, physical, and biological methods have been adopted to achieve the intended result, as enumerated in many pieces of literature. Therefore, selecting an efficient synthesis process is essential, which is a key factor that significantly influences the efficacy of the synthesized nanocrystalline materials. The chemical synthesis of nanoparticles (NPs) via hydrothermal, solvothermal, and sol-gel routes is considered effective as high-quality crystalline structures are produced. Control of parameters of processes yields excellent morphological features of the synthesized samples. This review explored the different parameters of processes and their effect on the morphology of $\mathrm{ZnO}$ nanostructures via hydrothermal, solvothermal, and sol-gel techniques. Finally, some $\mathrm{ZnO}$ nanocomposites molecules are reviewed as per the dopant used and its effect on the sample compound synthesized.
\end{abstract}

Keywords: synthesis methods; nanostructures; nanomaterials; nanoparticles; zinc oxide; morphology.

(C) 2021 by the authors. This article is an open-access article distributed under the terms and conditions of the Creative Commons Attribution (CC BY) license (https://creativecommons.org/licenses/by/4.0/).

\section{Introduction}

Zinc oxide is one example of transition metal oxides that exhibit unique electrical, optical and mechanical properties. Due to their unique properties, they have numerous applications in fields such as pharmaceuticals, electronics, consumer goods, optical and electrical devices, and environmental remediation [1-5]. The morphology of $\mathrm{ZnO}$ nanostructures has a significant role in their applications, according to Jin and Jin 2019 [6]. For instance, rod-like, sheet-like, and belt-like nanostructures are suitable for solar cells, lightemitting diodes, gas sensors, and biological probes. Interestingly, researchers focus on the morphology of nano $\mathrm{ZnO}$ to achieve their intended purpose in its applications. Controlling the size and shape of these nanostructures during synthesis is the major focus of the researcher.

Different synthesis techniques have been developed to be grouped into physical, chemical, and biogenic (green route). Physical techniques include pulsed laser deposition, magnetron sputtering, electrodeposition, and electron beam evaporation [7-11]. The chemical synthesis route comprises hydrothermal, solvothermal, sol-gel, chemical bath deposition, wet chemical process, spray pyrolysis, microemulsion, and precipitation methods [12-18]. Green 
or biogenic synthesis of metal oxide nanomaterials is plant or microbe mediated, with the extracts being biocompatible and can serve as capping agents for stabilizing the NPs [19, 20]. Although chemical synthesis poses toxicity to the environment, pure desired crystals with high stability and high yield are produced [21].

$\mathrm{ZnO}$ nanostructures morphology and size of $\mathrm{ZnO}$ nanostructures can be modified when reaction conditions (precursor and their concentrations, temperature, solvent type, and surfactants) in a particular synthesis route are controlled [22]. It was reported that, although different treatment methods and surfactants were used in the synthesis of $\mathrm{ZnO}$ nanostructures, similar flower-like structures were obtained using the same precursor (zinc acetate dihydrate) [23-24]. The use of surfactants (HMT and PEG 400) and annealing the synthesized ZnO nanocrystalline powder at $500{ }^{\circ} \mathrm{C}$ by Rocha et al. [23] resulted in the particle to record crystallite size of $30 \mathrm{~nm}$ as against free surfactant and without extra heat at a crystallite size of 7-8 nm. In another study by Vahidi et al. [25], ZnO NPs of different crystallite sizes (51, 60, and $61 \mathrm{~nm}$ ) but similar aggregated spherical shaped particles were obtained using Pelargonum zonale leaf extract and zinc nitrate as a precursor under different heating conditions (conventional heating, autoclave, and microwave irradiation). However, samples from the conventional heating process recorded the highest antibacterial effect of the bacterial strains used for the study. When this study was compared to that of Satheshkumar et al. [26], although the same morphology of aggregated spherical shaped particles was produced from the conventional heating process, the crystallite size was far below (1.62-1.88 nm) that of Vahidi et al. These variations may be attributed to different conditions such as the concentrations of the precursor and extracts used as well as the reaction conditions under which each sample was prepared. These reports indicate that variation of synthesis parameters could affect the morphology of crystals produced.

Literature suggests that different techniques are being developed for a suitable and easy means to achieve desired properties of these nanostructures. However, it was reported that wet techniques of synthesizing metal oxide nanomaterials usually produce impure particles with larger particle sizes [27]. These limitations result from the complexity of the chemical reactions during the growth and nucleation stages of the reaction mechanism, which makes it impossible for repeatability. Therefore, selecting an efficient synthesis process is essential, which is a key factor that significantly influences the efficacy of the synthesized nanocrystalline materials. This review critically examines the current development of appropriate reaction conditions that could be employed in four chemical synthesis methods (hydrothermal, solvothermal (microwave-assisted), sol-gel and hybrids) to achieve the desired features of zinc oxide nanoparticles needed for its specific application.

\section{ZnO Nanoparticles Synthesis by Hydrothermal Route}

This route of synthesizing $\mathrm{ZnO}$ nanoparticles involves using high heat and pressure (100-1000 ${ }^{\circ} \mathrm{C}$ and 1-10,000 atm) with water as a solvent in an autoclave (Figure 1). Although good quality crystals are produced, high energy consumption in some cases coupled with expensive autoclaves is some disadvantages to this technique. The heating method may be conventional or microwave, with each having its advantages and disadvantages. Other parameters such as the type of reactant, alkaline source, doping material, surfactant, or reaction conditions (calcination temperature and reaction time) determine the characteristics of nano $\mathrm{ZnO}$ synthesized. Conventional heating employs the principle of conduction and convection within the reaction medium resulting in the non-uniformity of heat to the molecules. Molecules 
are subjected to heat sources via two modes; First, where there is direct contact with the reacting molecules and the heat source element. And secondly, the heating element may be in contact with the containing vessel of the reaction mixture in the case of a heating mantle. The former may result in contaminations by the heating element in direct contact with the mixture. However, the latter is mostly preferred. This effect subsequently affects the nature and quality of samples synthesized. In furtherance to this, there is a high loss of heat as a result of the long duration of the heating process. Also, 'wall effect' is a phenomenon where the maximum temperature is experienced at the reaction vessel walls, leading to the heterogeneity of the obtained products. However, microwave irradiation is characterized by a short heating time with the heating directed to the molecules (precursor and solvent) by microwaves' energy, thereby providing intense friction and collision between the molecules, which accelerates the nucleation process [28]. Microwave heating ensures the synthesis of pure samples as there is uniformity in the heating of the molecules [29, 30]. For instance, the capability of solvent molecules to be irradiated at a given temperature and frequency to heat energy known as loss factor (loss factor of deionized water is 0.123 ) renders microwave heating more efficient in the hydrothermal method of synthesis [31]. However, impurities in the solvent decrease the penetration depth, according to Kim et al. [32]. These factors indicate that reaction molecules have acquired an electric dipole moment in proportion to the applied electric field.

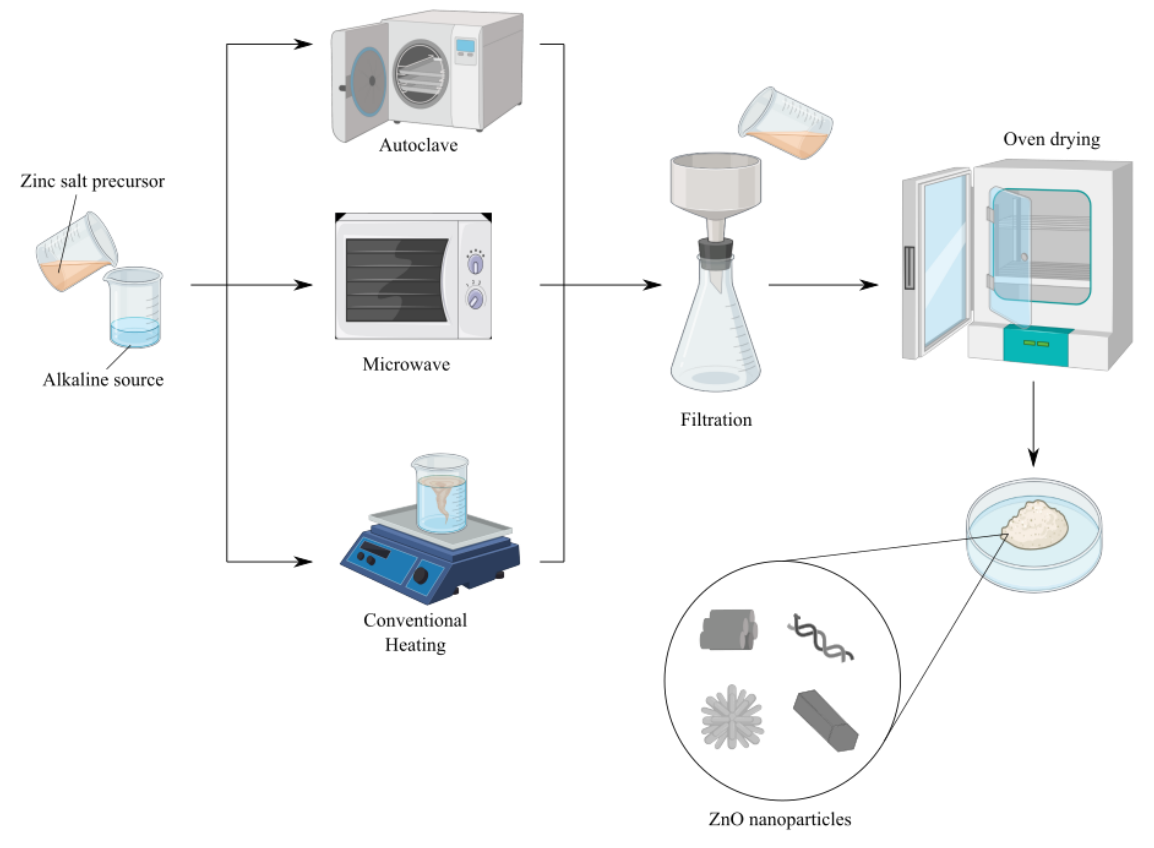

Figure 1. Overview of the hydrothermal method to synthesize $\mathrm{ZnO}$ nanoparticles.

$\mathrm{ZnO}$ nanocrystals were synthesized using autoclave mode of heating at temperatures between $100-150{ }^{\circ} \mathrm{C}$ for $1-5$ hours by Vellakkat et al. [33]. The study reported that variation of reaction temperature and time produced diverse morphologies (flower-like, rod-like, and spherical granular). The study also reported that an increase in temperature time decreased the crystallite size $(46.19,41.66$, and $34.74 \mathrm{~nm})$, whereas an increase in reaction time increased the size $(44.76,53.51$, and $78.21 \mathrm{~nm})$. Meanwhile, high purity hexagonal wurtzite $\mathrm{ZnO}$ nanocrystals were produced from microwave-assisted hydrothermal technique by Ming et al. [34]. From their study, a mixture of zinc nitrate, potassium sodium citrate, and sodium hydroxide solutions was irradiated in a microwave oven $(650 \mathrm{~W}, 2.45 \mathrm{GHz})$ for 20 minutes at $90{ }^{\circ} \mathrm{C}$. High resolution of the spherical surface features of the particles revealed numerous 
interconnected nanosheets, which were displayed as microflower in the TEM analysis. In addition, the synthesized sample exhibited a high photocatalytic performance for Rhodamine B degradation due to its smaller band gap.

According to Suwanboon et al. [35], the type of precipitating agent (alkaline source) used influences the morphology of the $\mathrm{ZnO}$ nanocrystals produced. In their study, hexagonal columnar structures changed to hexagonal platelet structures with crystallite size (55.8-57.8 $\mathrm{nm}$ ) as the molar concentration of $\mathrm{LiOH}$ increased from 0 to 2 when the reaction mixture was autoclaved at $180{ }^{\circ} \mathrm{C}$ for 15 . However, $\mathrm{NaOH}$ produced rod-like structures (Figure 2a) with crystallite size $(46.7-48.0 \mathrm{~nm})$ under the same conditions. The study concluded that, whereas hexagonal $\mathrm{ZnO}$ platelets (Figure 2b) showed better photocatalytic efficiency to methyl blue, the rod-like structures exhibited better inhibitory activity against Staphylococcus aureus.

The effect of surfactants on the morphology of $\mathrm{ZnO}$ nanocrystals was investigated by Zhu et al. [36]. They reported that an increase in the concentration of CTAB (0.03-0.1 M) saw plate-like nanostructures aggregated to form flower-like structures (Figure 2c). A further increase in CTAB to $0.3 \mathrm{M}$ collapsed the flower-like structures. The synthesis conditions were reaction time of 3 hours, autoclave temperature of $150{ }^{\circ} \mathrm{C}$ for 16 hours, and calcination temperature of $500{ }^{\circ} \mathrm{C}$ for 2 hours. These flower-like nanostructures have been investigated as suitable materials for Sulphur dioxide gas detection [37] and ethanol sensing [36].

In other studies, the effects of reactants on the morphological structures of $\mathrm{ZnO}$ nanostructures were investigated. Perillo et al. [38] and Alver et al. [39] employed different zinc salt precursors $\left[\mathrm{Zn}\left(\mathrm{CH}_{3} \mathrm{COO}\right)_{2} \cdot 2 \mathrm{H}_{2} \mathrm{O} ; \quad \mathrm{ZnCl}_{2}\right.$ and $\left.\mathrm{Zn}\left(\mathrm{NO}_{3}\right)_{2} \cdot 6 \mathrm{H}_{2} \mathrm{O}\right]$ and hexamethylenetetramine (HMTA) at $90{ }^{\circ} \mathrm{C}$ for 6 hours on a magnetic stirrer and $140{ }^{\circ} \mathrm{C}$ for 4 hours in an autoclave respectively. HMTA acted as a $\mathrm{pH}$ buffer to release the $\mathrm{OH}^{-}$from the reaction mixture. Perillo et al. recorded hexagonal nano-rods with crystallite size (40-46 nm), particle length, and diameter (400 nm-6.5 $\mu \mathrm{m}$ and 73-300 nm) for all the precursors. Although Alver et al. also produced hexagonal rod-like nanostructures from zinc acetate and zinc nitrate precursors, zinc chloride produced hexagonal rod-like and plate-like structures (impurity). This impurity confirms the assertion made by Wojnarowicz et al. [27] that zinc chloride produces a stable by-product (simonkolleite), which is considered an impurity during the synthesis of $\mathrm{ZnO}$.
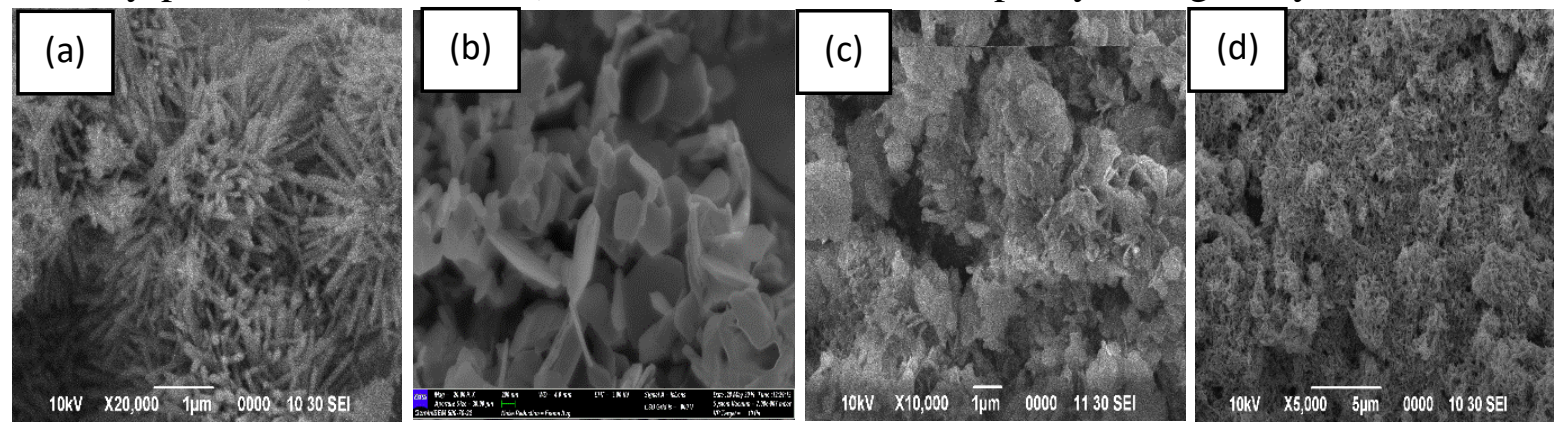

Figure 2. ZnO morphologies:(a) rod-like; (b) hexagonal platelets; (c) flower-like; (d) wire-like nanostructures.

Amin et al. [40] also investigated the effect of $\mathrm{pH}$, precursor concentration, growth time, and temperature on the morphology of $\mathrm{ZnO}$ nanostructures. They reported that tetrapodlike, flower-like, and urchin-like structures were obtained when the $\mathrm{pH}$ of the reaction mixture was from 8-12.5. However, as the $\mathrm{pH}$ was lowered from 8 to 4.6, rod-like structures eroded gradually to wire-like nanostructures (Figure 2d). Meanwhile, when the concentration of the precursor $\mathrm{Zn}\left(\mathrm{NO}_{3}\right)_{2} \cdot 6 \mathrm{H}_{2} \mathrm{O}$, was also decreased from $400-25 \mathrm{mM}$, the micro-rods observed at pH 8 gradually thinned out to wire-like structures with a diameter $(<100 \mathrm{~nm})$ and length $(1.2$ 
$\mu \mathrm{m})$. The average length of the rod-like structures produced was directly proportional to growth duration. The first 6 hours increased the length from $500 \mathrm{~nm}$ to $1.8 \mu \mathrm{m}$ until the final length increased to $2.2 \mu \mathrm{m}$ at the $10^{\text {th }}$ hour. There was no further increase in length after the 10 hours of duration. They also reported that the aspect ratio of the synthesized rod-like structures increased with increasing temperature from $50{ }^{\circ} \mathrm{C}$ to $95^{\circ} \mathrm{C}$ beyond which there was no change. Similarly, rod-like nanostructures were reduced to thin-film macrostructures when the precursor concentration was increased from $10 \mathrm{mM}$ to $500 \mathrm{mM}$ [41]. Table 1.0 illustrates other literature on hydrothermal synthesis using microwave-assisted and conventional approaches in $\mathrm{ZnO}$ nanostructures synthesis and their applications.

\subsection{ZnO nanoparticles synthesis by the solvothermal route.}

The solvothermal technique is a widely used technique that usually uses non-aqueous solvents under controlled temperature and pressure higher than atmospheric pressure [42]. This technique requires a simple setup (Figure 3), less expensive equipment, relatively low synthesis temperature to yield a large area of deposition [43]. The technique also allows the microstructural control of the particles produced and the dispersity of ZnO NPs [44]. Despite these advantages, some of the solvents used are very costly and are very toxic to the environment.

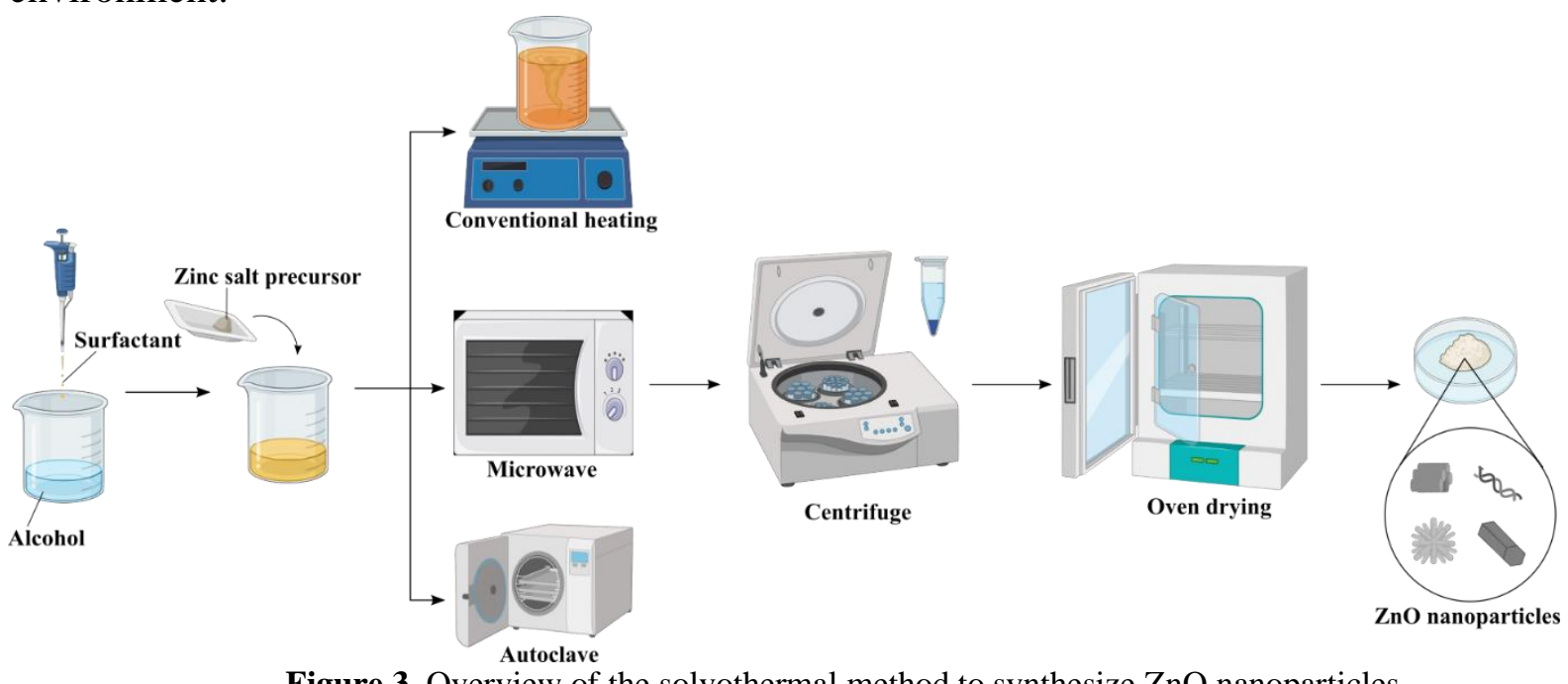

Figure 3. Overview of the solvothermal method to synthesize $\mathrm{ZnO}$ nanoparticles.

According to Šarić et al. [45], properties of the non-polar solvents enable the reaction mechanism and particle growth to be controlled easily as moisture to the system is eliminated. The intermediate species from the non-polar solvents used in this technique offer better characterization when analyzed with NMR spectroscopy compared to the aqueous system [46. These non-polar solvents can serve as surfactants. The type of non-polar solvent and its intermediate species formed during the synthesis process could affect the crystal growth as selectivity to the different facets of the $\mathrm{ZnO}$ crystal is possible [47].

In most solvothermal syntheses, ethanol and methanol (alcohol) are the most common solvents used due to their availability and cost. Other solvents, as well as surfactants used according to literature, including oleic acid, gluconic acid, tween 80 [48], ethylene glycol [4951], triethanolamine, TEA [43]. Surfactants are amphiphilic molecules classified as anionic, nonionic, cationic, or amphoteric in terms of charge present in the hydrophilic portion of the molecule after dissolution in an aqueous solution. They usually form water-in-oil microemulsions which may be of two or three categories; the first metal complex dissolved in the 
water pools, another metal salt complex, and the reducing agent. An example is the mixture of both platinum and palladium salts in the presence of sodium bis (2-ethylhexyl) sulfosuccinate (AOT) as surfactant and hydrazine as a reducing agent [52]. The surfactants tend to restrict the growth of the particles as the size of the emulsion, therefore, tend to determine the particle size. Šaric et al. [45] established that increase in the mole ratio of the surfactant and type of alcohol use increases the particle size. According to a review by Wojnarowicz et al. [27], surfactants such as Triton X100, polyethylene glycol 400 (PEG-400), polyvinylpyrrolidone (PVP) produces rod-like nanostructures [53], polyvinyl alcohol (PVA) results in the production of flake-like nanostructures [54], whiles polyvinylpyrrolidone (PVP) and cetyltrimethylammonium-bromide $(\mathrm{CTAB})$ produces flower-like nanostructures [55].

The reaction kinetics of the molecules are mainly governed by the transport of species through the heat transfer within the system. As indicated previously, thermal decomposition of reacting molecules is efficient when microwave irradiation is used compared to conventional heating. One microstructural property of $\mathrm{ZnO}$ NPs that influences their application is the agglomeration of the synthesized crystals. Literature has reported that Diethanolamine (DEA) and Triethanolamine (TEA) are suitable solvents used as polymerization and stabilization agents in controlling the morphology of $\mathrm{ZnO}$ structures by the aggregation of primary nanoparticles [43,56]. In a study by Saric et al. [45], Triethanolamine (TEA) was investigated to have an impact on the growth of particles. The result established that TEA acted as both a suppressor and modifier of the particles formed at a reaction temperature of $170{ }^{\circ} \mathrm{C}$. Furthermore, the growth and aggregation of the particles depended on the mole concentration ratio of the zinc precursor and TEA.

In other to develop the morphology of ZnO NPs to suit a particular application, Idiawati et al. [57] demonstrated the effect of growth time using a two-stage approach to grow $\mathrm{ZnO}$ nano-rods on Indium Tin Oxide (ITO) substrate. The first reaction stage (seed layer formation) employed zinc acetate dihydrate and Monoethanolamine (MEA) reaction at $70{ }^{\circ} \mathrm{C}$ for 2 hours. Then follows the growth stage where zinc nitrate tetrahydrate $\left[\mathrm{Zn}\left(\mathrm{NO}_{3}\right) .4 \mathrm{H}_{2} \mathrm{O}\right.$, Hexamethylenetetramine [HMT], and ITO were reacted at $90{ }^{\circ} \mathrm{C}$ with the growth-time varied at 4, 6, and 9 hours for each concentration. The as-prepared $\mathrm{ZnO}$ nanorods gave the crystallite size, nanorod length, and diameter to be $44-51 \mathrm{~nm}, 570-1280 \mathrm{~nm}$, and 138-236 nm, respectively. It has been proven that these rod-like nanostructures are showed resistance to wood decay fungus [58].

Nevertheless, Ming et al. [34] investigated the effect of different zinc salts (zinc sulfate, zinc nitrate, and zinc acetate) on Rhodamine B dye's morphology and photocatalytic property. The microwave operating conditions $(650 \mathrm{~W}, 2.45 \mathrm{GHz})$ produced microsphere structures covered with nanosheets and nanoparticles (nitrate and sulfate zinc) and microflower structures covered with nanosheets (zinc acetate). Nitrate-ZnO sample exhibited the best photocatalytic degradation of $93.63 \%$ after 120 minutes compared to acetate- $\mathrm{ZnO}$ and sulphate-ZnO samples with percentage degradation of $82.35 \%$ and $64.66 \%$, respectively. Other studies carried out using this route but with different conditions are illustrated in Table 2.0.

\subsection{ZnO nanoparticles synthesis by sol-gel route.}

Sol-gel as a chemical technique in metal oxide nanoparticle synthesis was a technology used to fabricate glass and ceramic materials some four decades ago, as reported by Dimitriev et al. [59]. The method is made up of aqueous and non-aqueous processes involving water and organic solvents. Aqueous sol-gel chemistry converts the precursor (mostly inorganic metal 
salt or metal alkoxides) into inorganic solid by virtue of the water molecules. On the other hand, non-aqueous sol-gel chemistry transforms the precursor (usually metal acetylacetonates and acetates, organometallic compounds, metal alkoxides, inorganic metal salts) via thermal decomposition. Sol-gel was adopted because of its considerable use of reduced temperature, which was economical compared to physical methods. This technique also offers the desired rate of thermal stability, good flexibility of crystal formation that is reproducible, better control of the particle size and shape to suit a wide range of applications, and relatively inexpensive apparatus set-up [60, 61]. Devoid these advantages, there are a few fundamental problems regarding the aqueous process, according to Livage et al. [62]. First, many reaction parameters ( $\mathrm{pH}$, precursor concentration, temperature, method of mixing, oxidation rate, hydrolysis, and condensation) need careful control to achieve the desired result. Also, the synthesized sample is generally amorphous, which needs the right annealing temperature to get the required crystalline structure. However, the non-aqueous process tends to overcome some of these major limitations of the aqueous process.

Sol-gel employs either a chemical solution (sol) or colloidal particles to produce an integrated network (gel) involving aqueous or organic solvent. The process involves various steps, including hydrolysis and polycondensation, gelation, aging, drying, and crystallization, as illustrated in Figure 4.

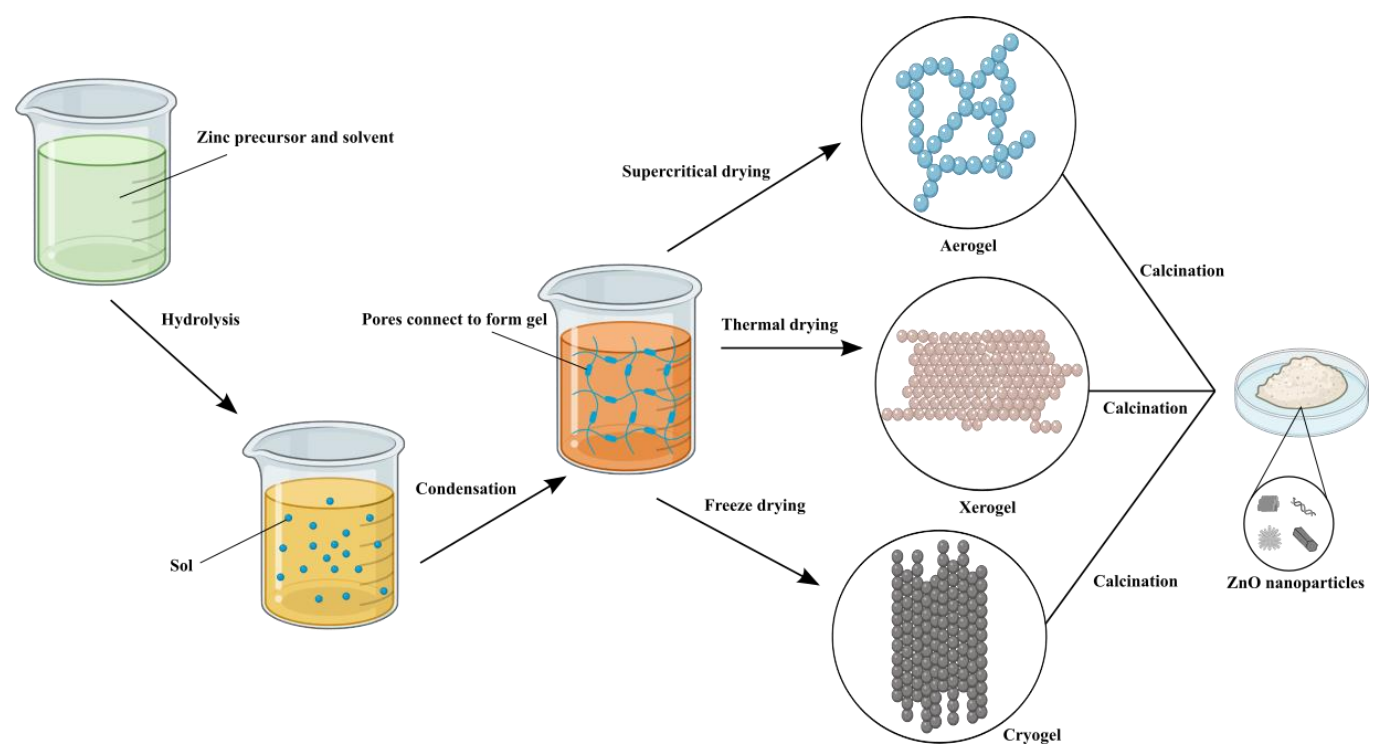

Figure 4. Overview of the sol-gel processes to synthesize $\mathrm{ZnO}$ nanoparticles.

Oxygen produced by the reaction solvent (water or alcohol) aids in forming the metal oxide in the hydrolysis stage. Alternatively, the use of an acid or alkaline source could also aid the hydrolysis of the precursor used. After the hydrolysis stage, the solvent condenses to form a colloidal mixture (gel), which yields hydroxyl (-OH) or oxo (-O) species to connect the metal molecules. The gelling process continues through the third stage until it is subjected to the type of drying, as illustrated in Figure 4. The type of drying process adopted affects the structure of the gel formed. Also, the nature of nanoparticles to be formed also depends on the drying process and relative humidity. For instance, nano-films may require low humidity for the stability of the sample formed. As already stated, calcination of the sample also influences the nature of the sample formed in terms of morphology $[64,65]$.

Shaikh and Ravangave [66] used the sol-gel method to synthesize nanorod structures with crystallite size in the range of 33-43 $\mathrm{nm}$ when zinc acetate and sodium hydroxide were 
used as starting materials. The reaction time was varied between $2-8$ hours, after which the asprepared samples were calcined at $100^{\circ} \mathrm{C}$ for 2 hours. Hasnidawani et al. [67] synthesized rodlike nanostructures with an average particle size of 81-85 $\mathrm{nm}$ with similar starting materials. Differences could be attributed to calcination and the longer reaction time employed by Shaikh and Ravangave.

According to Iwamura et al. [68], reaction time and purity of the nanomaterial can be enhanced with the use of the microwave-assisted sol-gel technique. Irradiation of zinc acetate dihydrate and $\mathrm{N}, \mathrm{N}$-dimethylacetoamide (DMAc) with a temperature of $64-118{ }^{\circ} \mathrm{C}$ for $1.5-4.0$ min duration yielded spherical nanocrystalline structures with a particle size of 312-509 nm. However, an increase in time to 6 min altered the morphology to nanowires of length and diameter, 1334 and $127 \mathrm{~nm}$, respectively. Also, the good and quality yield of cubic octamethylsilsesquioxane was synthesized using microwave-assisted technique [69].

The type of reagent used in sol-gel synthesis also influences the morphology as well as the optical properties of the synthesized samples. According to Vanaja and Rao [70], potassium and sodium hydroxide were used as precipitating agents on the precursor, zinc nitrate. Potassium hydroxide produced a smaller crystallite size $(21.59 \mathrm{~nm})$ of $\mathrm{ZnO}$ as compared to $36.89 \mathrm{~nm}$ when sodium hydroxide was used. Both agents produced irregular spherical-shaped structures with sizes 17-25 nm and 30-50 nm for $\mathrm{KOH}$ and $\mathrm{NaOH}$, respectively.

Apart from the solvent controlling the reaction mechanism in non-aqueous sol-gel processes, surfactants can also be used in the presence of the precursor in the transformation process, thereby acting as stabilizing ligands at temperature ranges of $250-350{ }^{\circ} \mathrm{C}$. The surfactant's ability to select the specific crystal face during the growth stage enables the control of the morphology and agglomeration as well as the surface properties of the nanoparticles. However, surface-adsorbed surfactant compromises the gas sensing effect or catalysis of some nanomaterials as well as their toxicity [71]. Thorn-like $\mathrm{ZnO}$ nanostructures were synthesized through the sol-gel method by Khan et al. [72], when zinc acetate, sodium hydroxide, and cetyltrimethylammonium bromide $(\mathrm{CTAB})$ was reacted under different stirring conditions $(500,1000,1500$, and $2000 \mathrm{rpm})$ for half an hour. Although other nanostructures such as nanoflowers, nanorods, nanowhiskers, nanobelts, nanotubes, nanorings, and nanocolumns were also identified, the thorn-like structures (Figure 5) were dominant with size determined at $<50 \mathrm{~nm}$ by TEM analysis.

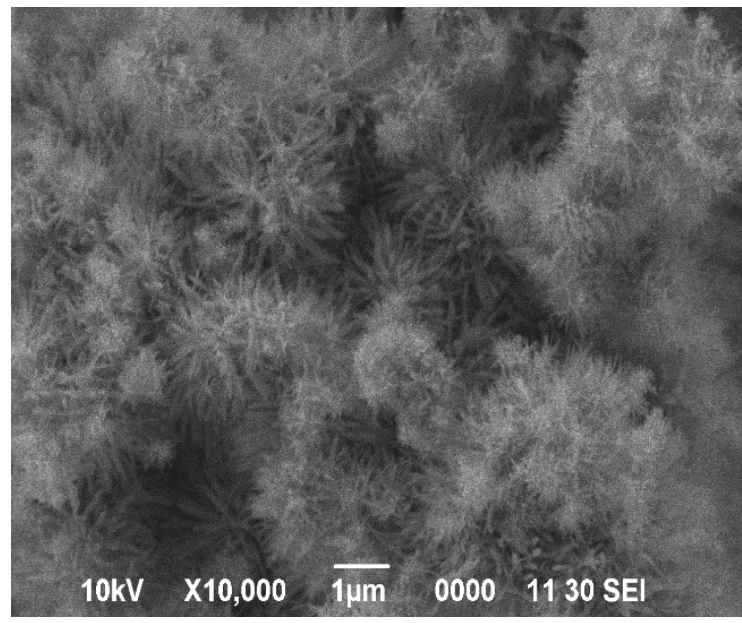

Figure 5. Thorn-like $\mathrm{ZnO}$ nanostructures.

Brintha and Ajitha [73] compared the morphological differences when $\mathrm{ZnO}$ nanostructures were prepared using sol-gel, hydrothermal and aqueous solution techniques. 
Although sol-gel employed high annealing temperature of $450{ }^{\circ} \mathrm{C}$ for 6 hours, the other techniques had their reaction temperatures between $80-100{ }^{\circ} \mathrm{C}$ for 6 hours. Hydrothermal and sol-gel techniques produced spherical and flower-like structures with $14 \mathrm{~nm}$ and $18 \mathrm{~nm}$ crystallite, respectively. On the other hand, the aqueous solution technique recorded a mixture of the two structures with a crystallite size of $13 \mathrm{~nm}$. Results from this study were similar to that of [74] when $\mathrm{ZnO}$ was doped with $\mathrm{Ni}$ and $\mathrm{Al}$ in a sol-gel process. Other studies carried out using the sol-gel method of synthesis with different conditions and useful application of the asprepared samples are illustrated in Table 3.0.

\subsection{ZnO hybrid/composite synthesis route.}

The intent of producing suitable changes in electrical, optical, and magnetic properties of metal oxide nanomaterials for their intended practical purposes is through the use of some selected elements in the periodic table (Group I, II, III, transition metals, metalloids, nonmetals, and lanthanides) as doping materials [75]. Since these transition elements are semiconductors, creating defects in the lattice of these semiconductors or forming hybrid materials with their required properties helps make the fabricated composite material an effective photocatalyst. Elements that are suitable as dopants in $\mathrm{ZnO}$ nanostructures should have a small ionic radius as that of $\mathrm{Zn}(0.74 \AA)$. This enables the ions to easily migrate over the crystal to occupy the interstitial positions, thereby affecting the nanocomposite compound's structural, morphological, and optical properties. According to Yousefi et al. [76], Li-doped $\mathrm{ZnO}$ showed better crystal quality than $\mathrm{Na}$ and $\mathrm{K}$-doped $\mathrm{ZnO}$ as well as the undoped $\mathrm{ZnO}$. It was also evident that, when alkaline earth metals $\left(\mathrm{Mg}^{2+}, \mathrm{Ca}^{2+}, \mathrm{Sr}^{2+}\right.$, and $\left.\mathrm{Ba}^{2+}\right)$ was used as a dopant in a co-precipitation technique by Hameed et al. [77], XRD patterns of $\mathrm{Mg}$ and $\mathrm{Ca}-$ doped- $\mathrm{ZnO}$ showed no addition phase due to their ionic radii $(0.66 \AA$ \& $0.99 \AA$ ) been close to that of $\mathrm{Zn}$ where the others were far above $\left(1.13 \AA\right.$ for $\mathrm{Sr}^{2+}$ and $1.35 \AA$ for $\left.\mathrm{Ba}^{2+}\right)$. The doped samples showed better enhancement in the crystalline nature as compared to the undoped sample.

Transition metal (TM) doped $\mathrm{ZnO}$ nanostructures are explored due to the magnetic and electronic properties they exhibit as a result of the electron spins in the $3 \mathrm{~d}$-orbital. Composite nanostructures synthesized from these species have better applications in electrical, electronics, and magnetic devices as compared to the bulk. The ionic radius of TMs was established to be one of the determining parameters for producing a very pure doped nanocrystalline structure. Zak et al. [78] identified $\mathrm{Ni}, \mathrm{Mn}$, and Co-doped $\mathrm{ZnO}$ to show a decreased particle size compared to the undoped. This result was attributed to the similar ionic radius of the dopants, $\mathrm{Ni}(0.69 \AA)$, Co $(0.745 \AA)$, and Mn $(0.46 \AA)$ to $\mathrm{Zn}$, where their lattice strain was replaced by $\mathrm{Zn}$ in the lattice structure.

From a study by Kaur et al. [79], Gd-doped-ZnO exhibited a paramagnetic behavior with a weak ferromagnetic component at low Gd-doping compared to the undoped. The doped sample was synthesized using zinc acetate dihydrate and gadolinium nitrate in 2-methoxy ethanol as solvent at $60{ }^{\circ} \mathrm{C}$ for $2 \mathrm{~h}$. After adding monoethanolamine, the sol was aged $24 \mathrm{~h}$ at room temperature, dried at $500{ }^{\circ} \mathrm{C}$ for $1 \mathrm{~h}$, and finally annealed at $500{ }^{\circ} \mathrm{C}$ for another $1 \mathrm{~h}$. Results from this study showed the formation of rod-like nanostructures with an average crystallite size of 25-37 nm. These hybrid nanostructures can be synthesized using different techniques. Table 4.0 illustrates doped- $\mathrm{ZnO}$ nanostructures synthesized by different techniques. 
Table 1. Characteristics of $\mathrm{ZnO}$ nanostructures synthesized via different hydrothermal routes.

\begin{tabular}{|c|c|c|c|c|c|}
\hline Method of synthesis & Precursor/Reactant & Synthesis condition & Properties & Applications & Reference \\
\hline $\begin{array}{l}\text { Microwave-assisted } \\
\text { without extra heat } \\
\text { treatment }\end{array}$ & $\begin{array}{l}\mathrm{Zn}(\mathrm{Ac})_{2} \cdot 2 \mathrm{H}_{2} \mathrm{O} \\
\mathrm{NH}_{4} \mathrm{OH} / \mathrm{NaOH}, \text { AOT }\end{array}$ & $\begin{array}{l}\text { Reaction time: } 10 \mathrm{~min} \text {; Autoclave } \\
\text { temp: } 80,100,120 \text { or } 140^{\circ} \mathrm{C} \text { for } 5,10 \text {, } \\
20 \mathrm{~min} \text {; Microwave power: } 300,600 \\
\text { and } 1200 \mathrm{~W} \text {; Drying time: } 65^{\circ} \mathrm{C} \text { for } 3 \\
\text { h. }\end{array}$ & $\begin{array}{l}\text { Hexagonal prism-like structures; Particle } \\
\text { size: } 200-300 \mathrm{~nm}\end{array}$ & & [80] \\
\hline $\begin{array}{l}\text { Microwave-assisted + } \\
\text { heat treatment }\end{array}$ & $\begin{array}{l}\mathrm{Zn}(\mathrm{Ac})_{2} \cdot 2 \mathrm{H}_{2} \mathrm{O}, \mathrm{N}_{2} \mathrm{H}_{4} \\
\mathrm{Zn}\left(\mathrm{NO}_{3}\right)_{2} \cdot 6 \mathrm{H}_{2} \mathrm{O}, \mathrm{NaOH} \\
\mathrm{NH}_{3}\end{array}$ & $\begin{array}{l}\text { Irradiation: } 15 / 10 \mathrm{~min} 510 / 680 \mathrm{~W} \text {; } \\
\text { Drying: } 100^{\circ} \mathrm{C} 2 \mathrm{~h} \text {; Irradiation: } 15 \\
\text { min } 150 \mathrm{~W} \text {; Drying: } 100{ }^{\circ} \mathrm{C} 2 \mathrm{~h} \text {; } \\
\text { Calcination temp: } 600^{\circ} \mathrm{C} 3 \mathrm{~h}\end{array}$ & $\begin{array}{l}\text { Needle-like, Flower-like, Spherical: } \\
\text { Particle diameter: } 50-150 \mathrm{~nm}\end{array}$ & & [81] \\
\hline $\begin{array}{l}\text { Microwave-assisted } \\
\text { without extra heat } \\
\text { treatment }\end{array}$ & $\begin{array}{l}\mathrm{Zn}(\mathrm{Ac})_{2} \cdot 2 \mathrm{H}_{2} \mathrm{O}, \mathrm{NaOH} \\
(\text { solvent }=\text { water/ethyl } \\
\text { alcohol; } 50 / 50 \mathrm{v} / \mathrm{v} \& 100 / 0 \\
\text { v/v) }\end{array}$ & $\begin{array}{l}\text { Reaction temp: } 100^{\circ} \mathrm{C} \& 140{ }^{\circ} \mathrm{C} \text { for } \\
45 \text { and } 60 \text { min; Microwave power: } 800 \\
\text { W; Drying temp: } 80^{\circ} \mathrm{C} \text { overnight; } \mathrm{pH} \\
=10\end{array}$ & $\begin{array}{l}\text { Plate-like, rounded plate-like, brush-like, } \\
\text { and flower-like; Band gap: } 3.17-3.24 \mathrm{eV} \text {; } \\
\text { Surface area: } 8.46-10.70 \mathrm{~m}^{2} / \mathrm{g}\end{array}$ & $\begin{array}{l}\text { Photocatalyst against } \\
\text { Rhodamine-B }\end{array}$ & [82] \\
\hline $\begin{array}{l}\text { Microwave-assisted } \\
\text { without extra heat } \\
\text { treatment }\end{array}$ & $\mathrm{Zn}\left(\mathrm{NO}_{3}\right)_{2}, \mathrm{NaOH}$ & $\begin{array}{l}\mathrm{pH}=8.3 ; \text { Microwave irradiation: } 1-5 \\
\text { min; Drying temp: } 70{ }^{\circ} \mathrm{C}\end{array}$ & $\begin{array}{l}\text { Rod-like, flower-like; Particle size } \\
\text { (diameter): 100-200 nm; Hydrodynamic } \\
\text { size (DLS): } 135-361 \mathrm{~nm}\end{array}$ & $\begin{array}{l}\text { Bio-imaging and drug } \\
\text { (Quercetin) delivery }\end{array}$ & [83] \\
\hline $\begin{array}{l}\text { Microwave-assisted } \\
\text { without extra heat } \\
\text { treatment }\end{array}$ & $\begin{array}{l}\mathrm{ZnCl}_{2}, \mathrm{NaOH}, \mathrm{CTAB}, \\
\text { Pluronic F127 }\end{array}$ & $\begin{array}{l}\text { reaction temp: } 50{ }^{\circ} \mathrm{C} \text { for } 90 \mathrm{~min} \text {; } \\
\text { Microwave power: } 2.45 \mathrm{GHz} ; 130 \mathrm{~W} \\
\text { for } 5 \mathrm{~min} \text {; Drying temp: } 60^{\circ} \mathrm{C} \text { for } 24 \mathrm{~h}\end{array}$ & $\begin{array}{l}\text { Cone-like (surfactant free \& Pluronic } \\
\text { F127), Plate-like (CTAB); Surface area: } \\
\text { 15.5-24.8 } \mathrm{m}^{2} / \mathrm{g} \text {; Crystallite size: 19.6-21.0 } \\
\mathrm{nm} \text {; Particle size: } 92.8 \mathrm{~nm} \text {; } 58.1 \mathrm{~nm} \\
\text { (CTAB); } 80.2 \mathrm{~nm} \text { (Pluronic F127); Band } \\
\text { gap: } 3.36 \mathrm{eV} \text { (free surfactant and CTAB); } \\
\text { 3.34 eV (Pluronic F127) }\end{array}$ & $\begin{array}{l}\text { Photocatalyst in the } \\
\text { degradation of methyl } \\
\text { blue (MB) }\end{array}$ & [84] \\
\hline $\begin{array}{l}\text { Microwave-assisted } \\
\text { without extra heating }\end{array}$ & $\begin{array}{l}\text { ACF Fabric Preparation: } \\
\mathrm{ACF}+\mathrm{HNO}_{3} \\
\text { Growth of } \mathrm{ZnO} \text { : } \\
\mathrm{Zn}(\mathrm{Ac})+\mathrm{NH}_{4} \mathrm{OH} \text {, on } \mathrm{ACF} \\
\text { fabric }\end{array}$ & $\begin{array}{l}\text { Reaction time: } 1 \mathrm{~h} \text {; Drying temp: } 50 \\
{ }^{\circ} \mathrm{C} \text { (oven), } 100{ }^{\circ} \mathrm{C} \text { for } 3 \mathrm{~h} \text { (furnace) } \\
\text { Reaction time: } 3 \mathrm{~h} \\
\text { Microwave power: } 1120 \mathrm{~W}, 2450 \mathrm{MHz} \\
\text { for } 30 \text { min x } 3 \text { steps; } \\
\text { Drying temp: } 50{ }^{\circ} \mathrm{C} \text { for } 24 \mathrm{~h} ; \mathrm{pH}=10 \text { - } \\
11\end{array}$ & Rod-like structures & Removal of tetracycline & [85] \\
\hline $\begin{array}{l}\text { Microwave-assisted } \\
\text { without extra heating }\end{array}$ & $\begin{array}{l}\mathrm{Zn}(\mathrm{Ac})_{2} \cdot 2 \mathrm{H}_{2} \mathrm{O}, \mathrm{TEOA}, \\
\mathrm{BTCA}, \mathrm{KOH}\end{array}$ & $\begin{array}{l}\text { Microwave power: } 2.45 \mathrm{GHz}, 800 \mathrm{~W} \\
150^{\circ} \mathrm{C}, 30 \text { min; Drying temp: } 100{ }^{\circ} \mathrm{C} ; \\
\mathrm{pH}=12 / 13\end{array}$ & $\begin{array}{l}\text { Dumb-bell (pH 8), } \\
\text { Spherical (pH 9) } \\
\text { Hexagonal bi-pyramidal (pH 10); Surface } \\
\text { area: } 35,15,25 \mathrm{~m}^{2} / \mathrm{g} \text { (Dumb-bell, } \\
\text { spherical, hexagonal bi-pyramidal); } \\
\text { Particle size: 195, 430, } 60 \mathrm{~nm} \text { (Dumb- } \\
\text { bell, spherical, hexagonal bi-pyramidal) }\end{array}$ & Photoelectrode & [86] \\
\hline
\end{tabular}


https://doi.org/10.33263/BRIAC123.42614292

\begin{tabular}{|c|c|c|c|c|c|}
\hline Method of synthesis & Precursor/Reactant & Synthesis condition & Properties & Applications & Reference \\
\hline $\begin{array}{l}\text { Microwave-assisted } \\
\text { with extra heating }\end{array}$ & $\begin{array}{l}\text { ZnO nanorods synthesis } \\
\mathrm{Zn}(\mathrm{Ac})_{2} \cdot 2 \mathrm{H}_{2} \mathrm{O}, \mathrm{NaOH} \\
\left(\mathrm{Mole} \text { ratio: } \mathrm{Zn}^{2+}: \mathrm{OH}^{-}(1:\right. \\
0,3,6,9,12,15) \\
\text { ZnO photoanode synthesis } \\
\mathrm{ZnO}, \mathrm{PEG}, \mathrm{C}_{2} \mathrm{H}_{5} \mathrm{OH}\end{array}$ & $\begin{array}{l}\text { Microwave power: } 450 \mathrm{~W}, 5 \mathrm{~min} \text {; } \\
\text { Drying temp: } 80{ }^{\circ} \mathrm{C} \text { for } 24 \mathrm{~h} \\
\text { Reaction time: } 1.5 \mathrm{~h} \text {; Drying temp: } 80 \\
{ }^{\circ} \mathrm{C} \text { for } 30 \mathrm{~min} \text {; Calcination temp: } 450 \\
{ }^{\circ} \mathrm{C} \text { for } 1.5 \mathrm{~h}\end{array}$ & $\begin{array}{l}\text { Plate-like, flower-like structures; Energy } \\
\text { band gap: } 3.201-3.217 \mathrm{eV}\end{array}$ & $\begin{array}{l}\text { Photoanode, } \\
\text { Photocatalyst in the } \\
\text { degradation of methyl } \\
\text { blue (MB) }\end{array}$ & [87] \\
\hline $\begin{array}{l}\text { Microwave-assisted } \\
\text { without extra heating }\end{array}$ & $\mathrm{Zn}\left(\mathrm{NO}_{3}\right)_{2} \cdot 6 \mathrm{H}_{2} \mathrm{O}, \mathrm{NaOH}$ & $\begin{array}{l}\text { Microwave power: } 70-100 \mathrm{~W}, 100- \\
240{ }^{\circ} \mathrm{C}, 3-7 \mathrm{~min}, 9-49 \text { bars; Reactor } \\
\text { parameters: } 120-200^{\circ} \mathrm{C}, 30 \mathrm{~min}, 1-14 \\
\text { bars; Drying temp: } 50{ }^{\circ} \mathrm{C} \text { for } 2 \mathrm{~h}\end{array}$ & $\begin{array}{l}\text { Particle size: } 20-250 \mathrm{~nm} \text {; Crystallite size: } \\
\text { 22-30 nm; } \\
\text { Particle size: } 30-400 \mathrm{~nm} \text {; Crystallite size: } \\
30-32 \mathrm{~nm}\end{array}$ & & [88] \\
\hline $\begin{array}{l}\text { Microwave-assisted } \\
\text { without extra heating }\end{array}$ & $\begin{array}{l}\mathrm{Zn}\left(\mathrm{NO}_{3}\right)_{2} \cdot 6 \mathrm{H}_{2} \mathrm{O}, \mathrm{NaOH} \\
\text { Gum Arabic }\end{array}$ & $\begin{array}{l}\text { Microwave power I: } 350 \mathrm{~W} \text { for } 2 \mathrm{~min} \text {; } \\
\text { Microwave power II: } 350 \mathrm{~W} \text { for } 2,4,6 \\
\& 10 \text { min; Drying temp: } 80^{\circ} \mathrm{C} ; \mathrm{pH}= \\
10\end{array}$ & $\begin{array}{l}\text { Spherical; Crystallite size: } 25 \mathrm{~nm} \text { ( } 2 \mathrm{~min}) \text {; } \\
\text { Particle size (DLS): } 160-180 \mathrm{~nm}(2-6 \\
\text { min); } 225 \mathrm{~nm}(10 \mathrm{~min}) \text {; Particle size } \\
\text { (FESEM): } \\
\text { 20-40 nm (non-aggregate); } 150-200 \mathrm{~nm} \\
\text { (aggregates) }\end{array}$ & & [89] \\
\hline $\begin{array}{l}\text { Microwave-assisted } \\
\text { without extra heating }\end{array}$ & $\begin{array}{l}\text { Seed layer preparation: } \\
\mathrm{Zn}(\mathrm{Ac})_{2} \cdot 2 \mathrm{H}_{2} \mathrm{O}, \mathrm{C}_{3} \mathrm{H}_{8} \mathrm{O} \\
\text { Growth of } \mathrm{ZnO} \text { nanorod: } \\
\text { Substrate, } \mathrm{Zn}\left(\mathrm{NO}_{3}\right)_{2} \cdot 6 \mathrm{H}_{2} \mathrm{O} \text {, } \\
\text { HMTA }\end{array}$ & $\begin{array}{l}\text { Annealing temp: } 300{ }^{\circ} \mathrm{C} \text { for } 30 \mathrm{~min} \\
(3 \mathrm{x}) \text {; Microwave power I: } 900 \mathrm{~W} \text { for } 2 \\
\text { min; < } 50 \text { PSI; Microwave power II: } \\
100 \& 1600 \mathrm{~W}, 80^{\circ} \mathrm{C} \text {, for } 5 \& 15 \mathrm{~min}\end{array}$ & $\begin{array}{l}\text { Rod-like and rod-like thin film structures; } \\
\text { Nanorod diameter: } 40-50 \mathrm{~nm} \text { (one step), } \\
600 \mathrm{~nm} \text { (two-stem); Crystallite size: } 1-10 \\
\mu \mathrm{m} \text { (one step), } 200 \mathrm{~nm} \text { (two step) }\end{array}$ & Electronic switch & [90] \\
\hline $\begin{array}{l}\text { Microwave-assisted } \\
\text { without extra heating }\end{array}$ & $\begin{array}{l}\mathrm{Zn}\left(\mathrm{NO}_{3}\right)_{2} \cdot 6 \mathrm{H}_{2} \mathrm{O}, \mathrm{NaOH}(4, \\
5 \& 6 \mathrm{mmol} \text { of } \mathrm{NaOH})\end{array}$ & $\begin{array}{l}\text { Microwave power: } 110^{\circ} \mathrm{C} \text { for } 1 \mathrm{~h} \text {; } \\
\text { Drying temp: } 80^{\circ} \mathrm{C} \text { for } 24 \mathrm{~h}\end{array}$ & $\begin{array}{l}\text { Star-like and chrysanthemum flower-like } \\
\text { structures; Energy band gap: } 3.21,3.22 \& \\
3.24 \mathrm{eV} \text {; Crystallite size: } 26,24,21 \mathrm{~nm} \\
\text { respectively. }\end{array}$ & $\begin{array}{l}\text { Photocatalyst for } \\
\text { reduction of } \\
\text { chromium(VI) }\end{array}$ & [91] \\
\hline $\begin{array}{l}\text { Microwave-assisted } \\
\text { without extra heating }\end{array}$ & $\mathrm{Zn}(\mathrm{Ac})_{2} \cdot 2 \mathrm{H}_{2} \mathrm{O}, \mathrm{NaOH}$ & $\begin{array}{l}\text { Reaction temp: } 75^{\circ} \mathrm{C} \text { for } 2 \mathrm{~h} \text {; } \\
\text { Autoclave temp: } 100,125,150,175 \& \\
200^{\circ} \mathrm{C} \text { for } 8,12,16,20, \& 24 \mathrm{~h} ; \\
\text { Drying temp: } 75^{\circ} \mathrm{C} \text { for } 6 \mathrm{~h}\end{array}$ & $\begin{array}{l}\text { Spherical shape }(8-24 \mathrm{~h}) \text {, Spherical \& } \\
\text { rod-like }\left(100-200{ }^{\circ} \mathrm{C}\right) \text {; Crystallite size: } \\
31-38 \mathrm{~nm}(8-24 \mathrm{~h}) ; \\
27-35 \mathrm{~nm}\left(100-200{ }^{\circ} \mathrm{C}\right) \text {; Particle size } \\
\text { (HR-TEM): } 30-40 \mathrm{~nm} \text {; Energy band gap: } \\
\text { 2.90-3.78 eV }\end{array}$ & & [92] \\
\hline $\begin{array}{l}\text { Microwave-assisted } \\
\text { without extra heating }\end{array}$ & $\mathrm{Zn}\left(\mathrm{NO}_{3}\right)_{2} \cdot 6 \mathrm{H}_{2} \mathrm{O}, \mathrm{NaOH}$ & $\begin{array}{l}\text { Microwave power: } 2.45 \mathrm{GHz}, 1000 \\
\mathrm{~W}, 10 \text { min; Drying temp: } 60^{\circ} \mathrm{C} \\
\text { overnight; } \mathrm{pH}=10\end{array}$ & $\begin{array}{l}\text { Spindle-like; Crystallite size: } \\
21 \mathrm{~nm} \text {; Surface area: } 11.06 \mathrm{~m}^{2} / \mathrm{g}(\mathrm{ZnO})\end{array}$ & $\begin{array}{l}\text { Antidiabetic \& } \\
\text { antibacterial inhibitor }\end{array}$ & [93] \\
\hline $\begin{array}{l}\text { Microwave-assisted } \\
\text { without extra heating }\end{array}$ & $\mathrm{Zn}\left(\mathrm{NO}_{3}\right)_{2}, \mathrm{NaOH}$ & $\begin{array}{l}\text { Irradiation time: } 15 \mathrm{~min} \text {; Drying temp: } \\
60^{\circ} \mathrm{C} \text { for } 24 \mathrm{~h} ; \mathrm{pH}=10\end{array}$ & $\begin{array}{l}\text { Spherical shape; Crystallite size: } \\
15.5 \mathrm{~nm}(\mathrm{ZnO})\end{array}$ & Antidiabetic activity & [94] \\
\hline $\begin{array}{l}\text { Microwave-assisted } \\
\text { without extra heating }\end{array}$ & $\begin{array}{l}\text { Seed layer preparation: } \\
\mathrm{Zn}(\mathrm{Ac})_{2} \cdot 2 \mathrm{H}_{2} \mathrm{O}, \mathrm{C}_{3} \mathrm{H}_{8} \mathrm{O}\end{array}$ & $\begin{array}{l}\text { Annealing temp: } 310^{\circ} \mathrm{C} \text { for } 60 \mathrm{~min} \\
\text { Microwave Power: } 2.45 \mathrm{GHz}, 105^{\circ} \mathrm{C} \\
\text { for } 5,10 \& 15 \mathrm{~min} \text {; Drying: } \mathrm{N}_{2} \text { gas }\end{array}$ & $\begin{array}{l}\text { Rod-like; Particle diameter \& length: 26- } \\
32 \mathrm{~nm} \& 440 \mathrm{~nm}(75 \mathrm{mM}), 35-39 \mathrm{~nm} \&\end{array}$ & & [95] \\
\hline
\end{tabular}




\begin{tabular}{|c|c|c|c|c|c|}
\hline Method of synthesis & Precursor/Reactant & Synthesis condition & Properties & Applications & Reference \\
\hline & $\begin{array}{l}\text { Precursor growth: } \\
\mathrm{Zn}\left(\mathrm{NO}_{3}\right)_{2} \cdot 6 \mathrm{H}_{2} \mathrm{O}(75 \mathrm{mM} \\
0.1 \mathrm{M}, 0.2 \mathrm{M}), \mathrm{C}_{6} \mathrm{H}_{12} \mathrm{~N}_{4}\end{array}$ & & $\begin{array}{l}600 \mathrm{~nm}(1.0 \mathrm{M}), 78-84 \mathrm{~nm} \& 1.5 \mu \mathrm{m}(2.0 \\
\mathrm{M}) \\
22-26 \mathrm{~nm} \& 430 \mathrm{~nm}(0.1 \mathrm{M} @ 5 \mathrm{~min}), \\
35-40 \mathrm{~nm} \& 60 \mathrm{~nm}(0.1 \mathrm{M} @ 10 \mathrm{~min}), \\
35-39 \mathrm{~nm} \& 605 \mathrm{~nm}(0.1 \mathrm{M} @ 15 \mathrm{~min})\end{array}$ & & \\
\hline $\begin{array}{l}\text { Microwave-assisted } \\
\text { without extra heating }\end{array}$ & $\mathrm{Zn}(\mathrm{Ac})_{2} \cdot 2 \mathrm{H}_{2} \mathrm{O}, \mathrm{NaOH}$ & $\begin{array}{l}\text { Microwave Power: } 2450 \mathrm{MHz}, 700 \\
\mathrm{~W}, 6 \text { min; Drying temp: } 60^{\circ} \mathrm{C} \text { for } 4 \mathrm{~h} \text {; } \\
\mathrm{pH}=6,8, \& 10\end{array}$ & $\begin{array}{l}\text { Irregular sheet-like (pH 6), Agglomerated } \\
\text { uniform microstructures (pH } 8 \& 10) \\
\text { Particle size: } \sim 100 \mathrm{~nm}(\mathrm{pH} 8 \& 10)\end{array}$ & Antibacterial activity & [96] \\
\hline $\begin{array}{l}\text { Microwave-assisted } \\
\text { without extra heating }\end{array}$ & $\begin{array}{l}\text { Seed layer preparation: } \mathrm{Si} \text {, } \\
\mathrm{ZnO} \text { deposit by } \mathrm{RF} \\
\text { magnetron sputtering } \\
\text { Precursor growth: } \\
\mathrm{Zn}(\mathrm{Ac})_{2} \cdot 2 \mathrm{H}_{2} \mathrm{O}(0.01 \sim 0.04 \\
\mathrm{mol} / \mathrm{L}), \mathrm{C}_{6} \mathrm{H}_{12} \mathrm{~N}_{4}\end{array}$ & $\begin{array}{l}\text { Sputtering parameters: } 100 \mathrm{~W} \text { for } 100 \\
\mathrm{~s},<7 \times 10^{-4} \mathrm{~Pa} \text { and } 1.0 \mathrm{~Pa} \\
\text { Microwave Power: } 60-110^{\circ} \mathrm{C}, 5-40 \\
\text { min }\end{array}$ & Rod-like; Particle size (TEM): $90 \mathrm{~nm}$ & & [97] \\
\hline $\begin{array}{l}\text { Microwave-assisted } \\
\text { without extra heating }\end{array}$ & $\begin{array}{l}\text { Synthesis of 1-carboxy4- } \\
\text { methylpyridin-1-ium } \\
\mathrm{C}_{6} \mathrm{H}_{7} \mathrm{~N}, \mathrm{C}_{2} \mathrm{H}_{3} \mathrm{ClO}_{2} \\
\text { Synthesis of Dye; } 4 \text { 4-[(E)- } \\
\text { 2-(furan-2-yl) } \\
\text { ethenyl]pyridin-1-ium-1- } \\
\text { yllacetate } \\
\mathrm{C}_{6} \mathrm{H}_{7} \mathrm{NO}_{2}, \mathrm{C}_{5} \mathrm{H}_{5} \mathrm{O}_{2}, \\
\mathrm{C}_{5} \mathrm{H}_{4} \mathrm{O}_{2}, \mathrm{C}_{2} \mathrm{H}_{5} \mathrm{OH}, \mathrm{C}_{5} \mathrm{H}_{5} \mathrm{~N} \\
\mathrm{ZnO}_{\text {synthesis }} \\
\mathrm{Zn}(\mathrm{Ac})_{2} \cdot 2 \mathrm{H}_{2} \mathrm{O}, \mathrm{Dye}(1 \% \text { \& } \\
3 \%), \mathrm{NaOH}\end{array}$ & $\begin{array}{l}\text { Reaction time: } 2 \mathrm{~h} \text {; Refluxing temp: } \\
70^{\circ} \mathrm{C} \text { for } 6 \mathrm{~h} \text {; Microwave power: } 20 \\
\text { min; Drying temp: } 60^{\circ} \mathrm{C} \text { for } 8 \mathrm{~h}\end{array}$ & $\begin{array}{l}\text { Hexagonal wurtzite structure; Crystallite } \\
\text { size: } 30.6 \mathrm{~nm} \text { (uncapped); } 22.9 \mathrm{~nm}(3 \% \\
\text { dye-capped); Energy band gap: } 3.6 \mathrm{eV}\end{array}$ & Bactericidal agent & [98] \\
\hline $\begin{array}{l}\text { Microwave-assisted } \\
\text { without extra heating }\end{array}$ & $\begin{array}{l}\mathrm{Zn}(\mathrm{Ac})_{2} \cdot 2 \mathrm{H}_{2} \mathrm{O}, \mathrm{TRIS} \\
(20 \%)\end{array}$ & $\begin{array}{l}\text { Microwave power: } 300 \mathrm{~W} \text { for } 3 \mathrm{~min} \text {; } \\
\text { Drying temp: } 80^{\circ} \mathrm{C} \text { overnight }\end{array}$ & Spherical; Energy band gap: $3.49 \mathrm{eV}$ & Antifungal agent & [99] \\
\hline $\begin{array}{l}\text { Microwave-assisted } \\
\text { without extra heating }\end{array}$ & $\begin{array}{l}\mathrm{Zn}(\mathrm{Ac})_{2} \cdot 2 \mathrm{H}_{2} \mathrm{O}, \text { Phthalic } \\
\text { acid }(\mathrm{PA}) / \text { Isophthalic acid } \\
\text { (IPA)/ Terephthalic acid } \\
\text { (TPA) }\end{array}$ & $\begin{array}{l}\text { Microwave power: } 2.45 \mathrm{GHz}, 800 \mathrm{~W} \text {, } \\
150{ }^{\circ} \mathrm{C} \text { for } 30 \mathrm{~min} \text {; Drying temp: } 100 \\
{ }^{\circ} \mathrm{C} \text { for } 12 \mathrm{~h} ; \mathrm{pH}=7,10 \& 12\end{array}$ & $\begin{array}{l}\text { Platelet shape (SDA), star-like (TPA), } \\
\text { rod-like (IPA), plate-like (PA); Particle } \\
\text { size: 50-500 nm (free SDA), 100-150 nm } \\
\text { (presence of SDA) }\end{array}$ & & [100] \\
\hline $\begin{array}{l}\text { Microwave assisted } \\
\text { with extra heating }\end{array}$ & $\begin{array}{l}\mathrm{Zn}\left(\mathrm{NO}_{3}\right)_{2} \cdot 6 \mathrm{H}_{2} \mathrm{O}, \mathrm{NaOH} \\
(1: 15 \text { molar ratio), } \mathrm{PEG}\end{array}$ & $\begin{array}{l}\text { Microwave power: } 2.45 \mathrm{GHz}, 320 \& \\
480 \mathrm{~W} \text { for } 5 \mathrm{~s} \& 15 \mathrm{~s} \text {; Drying temp: } 80 \\
{ }^{\circ} \mathrm{C} \text { for } 24 \mathrm{~h} \text {; Calcination temp: } 450{ }^{\circ} \mathrm{C} \\
\text { for } 1 \mathrm{~h}\end{array}$ & $\begin{array}{l}\text { Needle-like }(320 \mathrm{~W}), \text { Rod-like }(480 \mathrm{~W}) \text {; } \\
\text { Particle size }(\mathrm{PEG} \text { free/PEG): < } 500 \mathrm{~nm} / \\
300 \mathrm{~nm} \text { length }(320 \mathrm{~W}), 2 \mu \mathrm{m} / 3 \mu \mathrm{m} \\
\text { length }(480 \mathrm{~W}) \text {; Energy band gap: } 3.24 \\
\text { Ev (PEG free), 3.10-3.23 eV (PEG) }\end{array}$ & $\begin{array}{l}\text { Photocatalyst in the } \\
\text { degradation of methyl } \\
\text { blue (MB) }\end{array}$ & [101] \\
\hline $\begin{array}{l}\text { Microwave-assisted } \\
\text { with extra heating }\end{array}$ & $\begin{array}{l}\mathrm{NaC}_{12} \mathrm{H}_{25} \mathrm{SO}_{4}, \mathrm{C}_{3} \mathrm{H}_{8} \mathrm{O} \\
\mathrm{NH}_{3}, \mathrm{Zn}(\mathrm{Ac})_{2} \cdot 2 \mathrm{H}_{2} \mathrm{O}\end{array}$ & $\begin{array}{l}\text { Reaction temp: } 80^{\circ} \mathrm{C} \text { for } 2 \mathrm{~h} \text {; } \\
\text { Microwave power: } 2.45 \mathrm{GHz}, 100-800\end{array}$ & $\begin{array}{l}\text { Fake-like }(0.12 \mathrm{~W} / \mathrm{g}) \text {, spherical }(0.12 \& \\
0.56 \mathrm{~W} / \mathrm{g}) ; \text { Crystallite size: } 38.84 \mathrm{~nm}\end{array}$ & $\begin{array}{l}\text { Photocatalyst in the } \\
\text { degradation of phenol }\end{array}$ & [102] \\
\hline
\end{tabular}




\begin{tabular}{|c|c|c|c|c|c|}
\hline Method of synthesis & Precursor/Reactant & Synthesis condition & Properties & Applications & Reference \\
\hline & & $\begin{array}{l}\mathrm{W} \text { for } 2 \mathrm{~h}, 0.12,0.37 \text { and } 0.56 \mathrm{~W} / \mathrm{g} \text {; } \\
\text { Calcination temp: } 550^{\circ} \mathrm{C} \text { for } 3 \mathrm{~h}\end{array}$ & $\begin{array}{l}(0.12 \mathrm{~W} / \mathrm{g}), 34.52 \mathrm{~nm}(0.37 \mathrm{~W} / \mathrm{g}), 31.08 \\
\mathrm{~nm}(0.56 \mathrm{~W} / \mathrm{g}) ; \text { Energy band gap: } 2.89 \\
\mathrm{eV}(0.12 \mathrm{~W} / \mathrm{g}), 2.39 \mathrm{eV}(0.37 \mathrm{~W} / \mathrm{g}), 2.26 \\
\mathrm{eV}(0.56 \mathrm{~W} / \mathrm{g}) ; \text { Surface area: } \\
13.10 \mathrm{~m}^{3} / \mathrm{g}(0.12 \mathrm{~W} / \mathrm{g}), 13.57 \mathrm{~m}^{3} / \mathrm{g}(0.37 \\
\mathrm{W} / \mathrm{g}), 14.35 \mathrm{~m}^{3} / \mathrm{g}(0.56 \mathrm{~W} / \mathrm{g})\end{array}$ & & \\
\hline $\begin{array}{l}\text { Microwave-assisted } \\
\text { with extra heating }\end{array}$ & $\begin{array}{l}\text { Synthesis of Polystyrene } \\
(P S) \text { template: } \\
\mathrm{C}_{8} \mathrm{H}_{8}, \mathrm{PVP}(2: 1 \mathrm{w} / \mathrm{w}), \\
\mathrm{C}_{2} \mathrm{H}_{5} \mathrm{OH}, \mathrm{Na}_{2} \mathrm{~S}_{2} \mathrm{O}_{8} \\
\text { Hollow } \mathbf{Z n O} \mathrm{NPs} \\
\text { synthesis: } \\
\mathrm{PS}, \mathrm{Zn}(\mathrm{Ac})_{2} \cdot 2 \mathrm{H}_{2} \mathrm{O}, \mathrm{NH}_{4} \mathrm{OH} \\
\text { Glutathione-hollow } \mathbf{Z n O} \\
\text { NPs synthesis: } \\
\text { Glu, h- } \mathrm{ZnO}(1: 1), \mathrm{CH}_{3} \mathrm{OH}\end{array}$ & $\begin{array}{l}\text { Reaction temp: } 70{ }^{\circ} \mathrm{C} \text { for } 4 \mathrm{~h} \text {; Drying } \\
\text { temp: } 27^{\circ} \mathrm{C} \text {; Microwave irradiation: } \\
120^{\circ} \mathrm{C} \text { for } 10 \mathrm{~min} \text {; Calcination temp: } \\
527^{\circ} \mathrm{C} \text {; Sonicate and stir mixture for } 1 \\
\mathrm{~h}\end{array}$ & $\begin{array}{l}\text { Hollow with cavities; Particle size } \\
\text { (TEM): } 35 \mathrm{~nm} ; 1483.50 \mathrm{~nm} \\
\text { (hydrodynamic diameter by DLS); Cavity } \\
\text { diameter: } 34 \mathrm{~nm} \text {; Surface area: } 17.1 \mathrm{~m}^{2} / \mathrm{g} \\
(\mathrm{h}-\mathrm{ZnO}), 12.3 \mathrm{~m}^{2} / \mathrm{g}(\text { Glu-h-ZnO) }\end{array}$ & Adsorbent & {$[103]$} \\
\hline $\begin{array}{l}\text { Microwave-assisted } \\
\text { with extra heating }\end{array}$ & $\begin{array}{l}\text { Seed layer preparation: } \\
\text { Glass substrate, } \\
\mathrm{Zn}(\mathrm{Ac})_{2} \cdot 2 \mathrm{H}_{2} \mathrm{O} \text { deposit by } \\
\text { spraying } \\
\text { Precursor growth: } \\
\text { Seeded substrate, } \\
\mathrm{Zn}\left(\mathrm{NO}_{3}\right)_{2} \cdot 6 \mathrm{H}_{2} \mathrm{O}, \mathrm{HMTA}\end{array}$ & $\begin{array}{l}\text { Deposit spraying temp: } 350^{\circ} \mathrm{C} \text {; } \\
\text { Microwave power: } 90^{\circ} \mathrm{C}, 180 \mathrm{~W} \text { for } \\
45 \text { min }(4 x) \text {; Drying temp: } 90^{\circ} \mathrm{C} \\
\text { Calcination temp: } 100 \text { and } 350^{\circ} \mathrm{C} \text { for } \\
1 \mathrm{~h}\end{array}$ & $\begin{array}{l}\text { Rod-like; Particle size: } \\
\sim 4.3 \mu \mathrm{m} \text { (ave. length) } \\
100 \mathrm{~nm} \text { (ave. diameter); Energy band gap: } \\
3.2 \mathrm{eV}\left(100{ }^{\circ} \mathrm{C}\right), 2.9 \mathrm{eV}\left(350^{\circ} \mathrm{C}\right)\end{array}$ & $\begin{array}{l}\text { Photocatalyst in the } \\
\text { degradation of phenol }\end{array}$ & [104] \\
\hline $\begin{array}{l}\text { Microwave-assisted } \\
\text { with extra heating }\end{array}$ & $\begin{array}{l}\text { Seed layer preparation: } \\
\text { Glass substrate, } \\
\mathrm{Zn}(\mathrm{Ac})_{2} \cdot 2 \mathrm{H}_{2} \mathrm{O}, \mathrm{C}_{2} \mathrm{H}_{7} \mathrm{NO}, \\
\mathrm{C}_{3} \mathrm{H}_{8} \mathrm{O}_{2} \text { deposit by spin } \\
\text { coating } \\
\text { Precursor growth: } \\
\text { Seeded substrate, } \\
\mathrm{Zn}\left(\mathrm{NO}_{3}\right)_{2} \cdot 6 \mathrm{H}_{2} \mathrm{O}, \mathrm{HMTA}\end{array}$ & $\begin{array}{l}\text { Reaction temp: } 60^{\circ} \mathrm{C} \text { for } 1 \mathrm{~h} \text {; Drying } \\
\text { temp: } 300^{\circ} \mathrm{C} \text { for } 10 \mathrm{~min} \text {; Calcination } \\
\text { temp: } 100-500{ }^{\circ} \mathrm{C} \text {; Microwave power: } \\
90^{\circ} \mathrm{C} \text {, for } 60 \mathrm{~min}\end{array}$ & $\begin{array}{l}\text { Rod-like; Crystallite size: } 9-25 \mathrm{~nm} \text { (seed } \\
\text { layer), } 38-56 \mathrm{~nm} \text { (nanorods); Particle } \\
\text { size: } 180-350 \mathrm{~nm} \text { (nanorod length); } \\
\text { Energy band gap: } 3.22-3.30 \mathrm{eV} \text { (seed } \\
\text { layer), 3.17-3.98 eV (nanorods) }\end{array}$ & UV Sensor & [105] \\
\hline $\begin{array}{l}\text { Microwave-assisted } \\
\text { with extra heating }\end{array}$ & $\begin{array}{l}\text { Seed layer preparation: } \\
\text { FTO substrate, } \\
\mathrm{Zn}(\mathrm{Ac})_{2} \cdot 2 \mathrm{H}_{2} \mathrm{O}, \mathrm{C}_{2} \mathrm{H}_{5} \mathrm{OH} \\
\text { deposit by spin coating } \\
\text { Precursor growth: } \\
\text { FTO substrate, } \\
\mathrm{Zn}\left(\mathrm{NO}_{3}\right)_{2} \cdot 6 \mathrm{H}_{2} \mathrm{O}, \mathrm{HMT}\end{array}$ & $\begin{array}{l}\text { Reaction temp: } 100^{\circ} \mathrm{C} \text { for } 15 \mathrm{~min} \text {; } \\
\text { Annealing temp: } 350^{\circ} \mathrm{C} \text { for } 1 \mathrm{~h} \text {; } \\
\text { Microwave power: } 1100 \mathrm{~W} \text { for } 20 \mathrm{~s} \\
\text { (microwave-assisted hydrolysis); } \\
\text { Oven temp: } 90^{\circ} \mathrm{C} \text { for } 45 \text { min } \\
\text { (hydrothermal process); Drying } \\
\text { samples with } \mathrm{N}_{2} \text { gas. }\end{array}$ & $\begin{array}{l}\text { Rod-like; Particle size: } 80 \mathrm{~nm} \text { (diameter } \\
\text { of the microwave-assisted sample), } 30 \mathrm{~nm} \\
\text { (diameter of the hydrothermal sample) }\end{array}$ & CO gas detector & {$[106]$} \\
\hline
\end{tabular}


https://doi.org/10.33263/BRIAC123.42614292

\begin{tabular}{|c|c|c|c|c|c|}
\hline Method of synthesis & Precursor/Reactant & Synthesis condition & Properties & Applications & Reference \\
\hline $\begin{array}{l}\text { Microwave-assisted } \\
\text { without extra heating }\end{array}$ & $\begin{array}{l}\mathrm{Zn}\left(\mathrm{NO}_{3}\right)_{2} \cdot 6 \mathrm{H}_{2} \mathrm{O}, \text { TEA } \\
(1: 30 \mathrm{v} / \mathrm{v})\end{array}$ & $\begin{array}{l}\text { Microwave power: } 640 \mathrm{~W} \text { for } 10 \mathrm{~min} \text {; } \\
\text { Vacuum drying }\end{array}$ & Crystallite size: $\sim 27 \mathrm{~nm}$ & $\begin{array}{l}\text { Photocatalyst in the } \\
\text { degradation of CTAB }\end{array}$ & [107] \\
\hline $\begin{array}{l}\text { Microwave-assisted } \\
\text { with extra heating }\end{array}$ & $\begin{array}{l}\mathrm{Zn}(\mathrm{Ac})_{2} \cdot 2 \mathrm{H}_{2} \mathrm{O}, \mathrm{NH}_{4} \mathrm{OH} \\
\mathrm{ZnCl}_{2}, \mathrm{Zn}\left(\mathrm{NO}_{3}\right)_{2} \cdot 6 \mathrm{H}_{2} \mathrm{O} \\
\mathrm{ZnSO}_{4} \cdot 7 \mathrm{H}_{2} \mathrm{O}\end{array}$ & $\begin{array}{l}\text { Microwave power: } 2450 \mathrm{MHz}, 640 \mathrm{~W} \\
\text { for } 10 \mathrm{~min} \text {; Vacuum drying: } 90^{\circ} \mathrm{C} \text { for } \\
12 \mathrm{~h} \text {; Calcination temp: } 600{ }^{\circ} \mathrm{C} \text { for } 2 \mathrm{~h} \text {; } \\
\mathrm{pH}=9\end{array}$ & $\begin{array}{l}\text { Flake-like (zinc acetate), Rod-like (zinc } \\
\text { nitrate), Spherical (zinc sulfate), } \\
\text { Hexagonal tubular (zinc chloride) }\end{array}$ & $\begin{array}{l}\text { Gas sensor for Volatile } \\
\text { Organic Compounds } \\
\text { (VOC) }\end{array}$ & [108] \\
\hline $\begin{array}{l}\text { Microwave-assisted } \\
\text { with extra heating }\end{array}$ & $\begin{array}{l}\mathrm{Zn}\left(\mathrm{NO}_{3}\right)_{2} \cdot 6 \mathrm{H}_{2} \mathrm{O}, \mathrm{HMT} \\
\mathrm{NaOH}\end{array}$ & $\begin{array}{l}\text { Microwave power: } 110,310, \& 710 \\
\mathrm{~W}, 90,150 \& 220^{\circ} \mathrm{C} \text { for } 15 \mathrm{~min} ; \\
\text { Calcination temp: } 200^{\circ} \mathrm{C} \text { for } 2 \mathrm{~h} ; \mathrm{pH} \\
=13\end{array}$ & $\begin{array}{l}\text { Plate-like; Crystallite size: } 22.40-24.83 \\
\mathrm{~nm} \text {; Energy band gap: } 3.28-3.38 ; \text { Surface } \\
\text { area: } 10.44-18.09 \mathrm{~m}^{2} / \mathrm{g} \text {; Particle size: } \\
\text { 400-600 nm (diameter) }\end{array}$ & & [109] \\
\hline $\begin{array}{l}\text { Microwave-assisted } \\
\text { without extra heating }\end{array}$ & $\begin{array}{l}\mathrm{Zn}\left(\mathrm{NO}_{3}\right)_{2} \cdot 6 \mathrm{H}_{2} \mathrm{O}, \text { PEG } 400 \\
\mathrm{NaOH} / \mathrm{NH}_{4} \mathrm{OH}\end{array}$ & $\begin{array}{l}\text { Microwave power: } 800 \mathrm{~W}, 100^{\circ} \mathrm{C} \text { for } \\
5 \text { min; Drying temp: } 110^{\circ} \mathrm{C} \text { for } 12 \mathrm{~h}\end{array}$ & 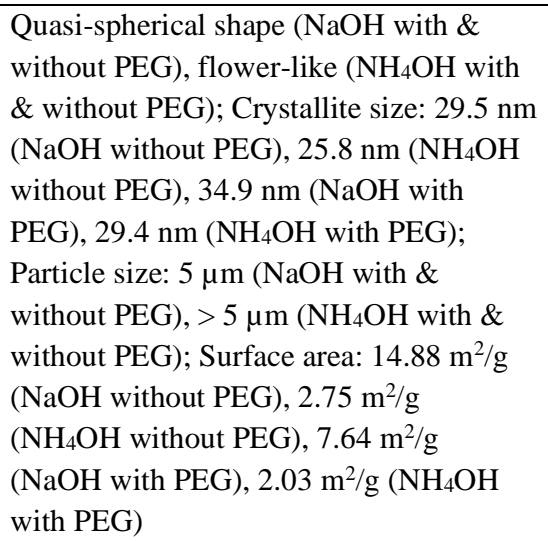 & $\begin{array}{l}\text { Catalyst for biodiesel } \\
\text { synthesis }\end{array}$ & [110] \\
\hline $\begin{array}{l}\text { Microwave-assisted } \\
\text { without extra heating }\end{array}$ & $\begin{array}{l}\text { Precursor growth process: } \\
\text { GaN substrate, } \\
\mathrm{Zn}(\mathrm{Ac})_{2} \cdot 2 \mathrm{H}_{2} \mathrm{O}, \mathrm{NaOH}\end{array}$ & $\begin{array}{l}\text { Microwave power: } 50^{\circ} \mathrm{C} \text { for } 2 \mathrm{~min} \\
\text { Drying temp: }<100^{\circ} \mathrm{C}\end{array}$ & $\begin{array}{l}\text { Rod-like; Particle size (height } \& \text { width): } \\
2.2 \mu \mathrm{m} / 2.5 \mu \mathrm{m}(\mathrm{pH}=6.75), 1.5 \mu \mathrm{m} / 0.7 \\
\mu \mathrm{m}(\mathrm{pH}=7.25), 1.5 \mu \mathrm{m} / 0.5 \mu \mathrm{m}(\mathrm{pH}= \\
7.75)\end{array}$ & & [111] \\
\hline $\begin{array}{l}\text { Microwave-assisted } \\
\text { with extra heating }\end{array}$ & $\mathrm{Zn}(\mathrm{Ac})_{2} \cdot 2 \mathrm{H}_{2} \mathrm{O}, \mathrm{KOH}$ & $\begin{array}{l}\text { Microwave power: } 1000 \mathrm{~W} \text { for } 0,0.5 \text {, } \\
1,1.5 \text { \& } 2 \text { min; Drying temp: } 80{ }^{\circ} \mathrm{C} \text {; } \\
\text { Calcination temp: } 400{ }^{\circ} \mathrm{C} \text { for } 1 \mathrm{~h}\end{array}$ & $\begin{array}{l}\text { Rod-like ( } 0 \mathrm{~min}) \text {, Tetrapod }(0.5 \mathrm{~min}) \text {, } \\
\text { Flower-like (1-2 min); Crystallite size: } \\
\text { 53, 59.3, } 69.2,74.5 \text { and } 82.4 \mathrm{~nm}(0-2 \\
\text { min); Particle size (length): } 255 \mathrm{~nm}(0.5 \\
\text { min), } 397 \mathrm{~nm}(1-2 \mathrm{~min}) \text {; Energy band } \\
\text { gap: } 3.24 \mathrm{eV}, 3.23 \mathrm{eV}, 3.21 \mathrm{eV}, 3.19 \mathrm{eV} \\
\text { and } 3.17 \mathrm{eV}(0-2 \mathrm{~min})\end{array}$ & $\begin{array}{l}\text { Photocatalyst for the } \\
\text { degradation of } \\
\text { rhodamine } \mathrm{B}(\mathrm{RhB})\end{array}$ & [112] \\
\hline $\begin{array}{l}\text { Microwave-assisted } \\
\text { without extra heating }\end{array}$ & $\begin{array}{l}\text { Seed layer preparation: } \mathrm{p}- \\
\mathrm{Si}(100) \text { substrate, } \\
\mathrm{Zn}\left(\mathrm{NO}_{3}\right)_{2} \cdot 6 \mathrm{H}_{2} \mathrm{O}, \mathrm{C}_{6} \mathrm{H}_{12} \mathrm{~N}_{4} \\
\text { deposit by } \mathrm{RF} \text { sputtering } \\
\text { Precursor growth: }\end{array}$ & $\begin{array}{l}\text { Annealing temp: } 400{ }^{\circ} \mathrm{C} \text { for } 1 \mathrm{~h} \text {; } \\
\text { Microwave power: } 90{ }^{\circ} \mathrm{C} \text { for } 2 \mathrm{~h} \text {; } \\
\text { Sampled dried with } \mathrm{N}_{2} \text { gas. }\end{array}$ & $\begin{array}{l}\text { Rod-like; Crystallite size: } 52.08 \mathrm{~nm} \text {; } \\
\text { Particle size (length): } 1 \mu \mathrm{m}\end{array}$ & UV detector & [113] \\
\hline
\end{tabular}




\begin{tabular}{|c|c|c|c|c|c|}
\hline Method of synthesis & Precursor/Reactant & Synthesis condition & Properties & Applications & Reference \\
\hline & $\begin{array}{l}\text { FTO substrate, } \\
\mathrm{Zn}\left(\mathrm{NO}_{3}\right)_{2} \cdot 6 \mathrm{H}_{2} \mathrm{O}, \mathrm{HMT}\end{array}$ & & & & \\
\hline $\begin{array}{l}\text { Microwave-assisted } \\
\text { with extra heating }\end{array}$ & $\begin{array}{l}\mathrm{Zn}\left(\mathrm{NO}_{3}\right)_{2} \cdot 6 \mathrm{H}_{2} \mathrm{O}, \mathrm{C}_{6} \mathrm{H}_{12} \mathrm{~N}_{4} \\
\text { (mole ratio of 3:20, 5:20, } \\
12: 20,20: 20 \text { and } 30: 20 \text { ) }\end{array}$ & $\begin{array}{l}\text { Microwave power: } 750 \mathrm{~W}, \sim 120^{\circ} \mathrm{C} \\
\text { for } 10 \text { min; Drying temp: } 80^{\circ} \mathrm{C} \text { for } 24 \\
\mathrm{~h} \text {; Calcination temp: } 400{ }^{\circ} \mathrm{C} \text { for } 1 \mathrm{~h}\end{array}$ & $\begin{array}{l}\text { Spherical to hexagonal rod-like } \\
\text { structures; Crystallite size: } 16.7-57.9 \mathrm{~nm} \text {; } \\
\text { Particle size: } 25 \mathrm{~nm} \text { to micro/sub micro } \\
\text { sizes }\end{array}$ & Antimicrobial agent & [114] \\
\hline $\begin{array}{l}\text { Microwave-assisted } \\
\text { without extra heating }\end{array}$ & $\begin{array}{l}\mathrm{Zn}(\mathrm{Ac})_{2} \cdot 2 \mathrm{H}_{2} \mathrm{O}, \mathrm{NH}_{4} \mathrm{OH} \\
(0.25,0.5,1.0,1.5,2.0 \text { or } \\
3.0 \mathrm{ml})\end{array}$ & $\begin{array}{l}\text { Microwave power: } 150{ }^{\circ} \mathrm{C} \text { for } 30 \mathrm{~min} \text {; } \\
\text { Drying temp: } 150{ }^{\circ} \mathrm{C} \text { for } 2 \mathrm{~h} \\
\mathrm{pH}=7-11\end{array}$ & $\begin{array}{l}\text { Hexagonal prism }(\mathrm{pH}=7) \text {, flower-like } \\
(\mathrm{pH}=11) ; \text { Particle size (width \& length): } \\
1 \& 5 \mu \mathrm{m}(\mathrm{pH}=7), 100 \mathrm{~nm}(\mathrm{pH}=8-10)\end{array}$ & $\begin{array}{l}\text { Photoelectrochemical } \\
\text { agent }\end{array}$ & {$[115]$} \\
\hline $\begin{array}{l}\text { Microwave-assisted } \\
\text { without extra heating }\end{array}$ & $\mathrm{Zn}(\mathrm{Ac})_{2} \cdot 2 \mathrm{H}_{2} \mathrm{O}, \mathrm{NaOH}$ & $\begin{array}{l}\text { Reaction temp: } 60^{\circ} \mathrm{C} \text { for } 2 \mathrm{~h} \text {; } \\
\text { Microwave power: } 90^{\circ} \mathrm{C} \text { for } 20 \mathrm{~min}\end{array}$ & Spherical; Particle size : $29 \mathrm{~nm}$ & & [116] \\
\hline
\end{tabular}

Table 2. Characteristics of $\mathrm{ZnO}$ nanostructures synthesized via different solvothermal routes.

\begin{tabular}{|c|c|c|c|c|c|}
\hline $\begin{array}{l}\text { Method of } \\
\text { synthesis }\end{array}$ & Precursor/Reactant & Synthesis condition & Properties & Applications & Reference \\
\hline $\begin{array}{l}\text { Solvothermal with } \\
\text { heat treatment }\end{array}$ & $\begin{array}{l}\mathrm{Zn}(\mathrm{Ac})_{2} \cdot \mathrm{H}_{2} \mathrm{O}, \text { ethanol/1- } \\
\text { propanol/1-butanol/1- } \\
\text { pentanol/1-octanol }\end{array}$ & Autoclave temp: $170^{\circ} \mathrm{C}$ for $4 \mathrm{~h}$ & $\begin{array}{l}\text { Spherical (1-butanol), rod-like (ethanol, 1- } \\
\text { propanol, 1-pentanol, 1-octanol); Particle } \\
\text { size: } 12 \mathrm{~nm}(1 \text {-butanol); Crystallite size: } \\
28 \mathrm{~nm} \text { (ethanol), } 14 \mathrm{~nm} \text { (1-propanol), } 12 \mathrm{~nm} \\
\text { (1-butanol), } 13 \mathrm{~nm} \text { (1-pentanol), } 18 \mathrm{~nm} \text { (1- } \\
\text { octanol) }\end{array}$ & & [117] \\
\hline $\begin{array}{l}\text { Microwave-assisted } \\
\text { with extra heat } \\
\text { treatment }\end{array}$ & $\begin{array}{l}\mathrm{Zn}\left(\mathrm{NO}_{3}\right)_{2} \cdot 6 \mathrm{H}_{2} \mathrm{O}, \mathrm{PVA}, \\
\text { Ascorbic acid }\end{array}$ & $\begin{array}{l}\text { Microwave power: } 2.45 \mathrm{GHz} \\
400 \mathrm{~W} \text { for } 3 \mathrm{~min} \text {; Drying temp: } \\
105^{\circ} \mathrm{C} \text { for } 3 \mathrm{~h} \text {; Calcination temp: } \\
500^{\circ} \mathrm{C} \text { for } 4 \mathrm{~h}\end{array}$ & $\begin{array}{l}\text { Spherical; Band gap: } 3.35 \mathrm{eV} \text {; Crystallite } \\
\text { size: } 20-22 \mathrm{~nm} \text {; Particle size: } \\
70-90 \mathrm{~nm} \text { (A), } 230-280 \mathrm{~nm}(\mathrm{~B}), 580-630 \mathrm{~nm} \\
\text { (C); Surface area: } 8.46-10.70 \mathrm{~m}^{2} / \mathrm{g}\end{array}$ & $\begin{array}{l}\text { Photocatalyst } \\
\text { against } \\
\text { Rhodamine-B } \\
\text { Antibacterial } \\
\text { agent }\end{array}$ & [118] \\
\hline $\begin{array}{l}\text { Solvothermal } \\
\text { synthesis with } \\
\text { conventional } \\
\text { heating }\end{array}$ & $\begin{array}{l}\text { Zinc salt, dimethyl sulfone, } \\
\mathrm{KOH}\end{array}$ & $\begin{array}{l}\text { Reaction temp: } 60^{\circ} \mathrm{C} \text { for } 3 \& 12 \\
\text { h; Drying temp: } 65^{\circ} \mathrm{C} \text { for } 12 \mathrm{~h}\end{array}$ & $\begin{array}{l}\text { Spherical; Crystallite size: } 4 \mathrm{~nm} \text { and } 10 \mathrm{~nm} \text {; } \\
\text { Particle size (TEM): } 4 \mathrm{~nm} \text { and } 10 \mathrm{~nm}\end{array}$ & $\begin{array}{l}\text { Antibacterial } \\
\text { agent }\end{array}$ & [119] \\
\hline $\begin{array}{l}\text { Solvothermal } \\
\text { synthesis with } \\
\text { conventional } \\
\text { heating }\end{array}$ & $\begin{array}{l}\mathrm{Zn}(\mathrm{Ac})_{2} \cdot 2 \mathrm{H}_{2} \mathrm{O}, \text { ethylene } \\
\text { glycol, } \mathrm{PVP}(0.001-0.004 \\
\text { mol })\end{array}$ & $\begin{array}{l}\text { Refluxing temp: } 195^{\circ} \mathrm{C} \text { for } 3 \mathrm{~h} \text {; } \\
\text { Drying temp: } 80^{\circ} \mathrm{C} \text { for } 2 \mathrm{~h} \text {; } \\
\text { Calcination temp: } 350{ }^{\circ} \mathrm{C} \text { for } 2 \mathrm{~h}\end{array}$ & $\begin{array}{l}\text { Spherical to wire-like; Crystallite size: } 14.6 \text {, } \\
\text { 12.8, } 47.0 \text { and } 81.0 \mathrm{~nm} \text {; Particle size: } 14.8, \\
\text { 12.5, } 47.0 \& 81.0 \mathrm{~nm} \text {; Particle length \& } \\
\text { diameter: } 20 \mu \mathrm{m} \& 22 \mathrm{~nm} \text {; Surface area: } 91 \text {, } \\
121,71 \& 53 \mathrm{~m}^{2} / \mathrm{g}\end{array}$ & Photoanodes & [120] \\
\hline $\begin{array}{l}\text { Solvothermal } \\
\text { synthesis with }\end{array}$ & $\begin{array}{l}\mathrm{Zn}(\mathrm{Ac})_{2} \cdot 2 \mathrm{H}_{2} \mathrm{O}, \mathrm{DMAc}: \mathrm{H}_{2} \mathrm{O} \\
(1: 0,4: 1,3: 2,2: 3,1: 4)\end{array}$ & $\begin{array}{l}\text { Reaction temp: } 95^{\circ} \mathrm{C} \text { for } 3 \mathrm{~h} \text {; } \\
\text { Drying temp: } 80^{\circ} \mathrm{C} \text { for overnight; } \\
\mathrm{pH}=6.12-6.86\end{array}$ & $\begin{array}{l}\text { Quasi-spherical, dumbbell shape, rod-like; } \\
\text { Ave. diameter: } 90 \mathrm{~nm}-0.7 \mu \mathrm{m} \text {; Energy band } \\
\text { gap: } 3.27-3.42 \mathrm{eV}\end{array}$ & & {$[121]$} \\
\hline
\end{tabular}




\begin{tabular}{|c|c|c|c|c|c|}
\hline $\begin{array}{l}\text { Method of } \\
\text { synthesis }\end{array}$ & Precursor/Reactant & Synthesis condition & Properties & Applications & Reference \\
\hline \multicolumn{6}{|l|}{$\begin{array}{l}\text { conventional } \\
\text { heating }\end{array}$} \\
\hline $\begin{array}{l}\text { Solvothermal } \\
\text { synthesis with } \\
\text { conventional } \\
\text { heating }\end{array}$ & $\begin{array}{l}\mathrm{Zn}(\mathrm{Ac})_{2} \cdot 2 \mathrm{H}_{2} \mathrm{O}, \text { ethanol, } \\
\mathrm{NaOH}, \mathrm{SDS} / \mathrm{PVP} / \mathrm{PEG}- \\
10000\end{array}$ & Reaction temp: $110^{\circ} \mathrm{C}$ for $10 \mathrm{~h}$ & $\begin{array}{l}\text { Spherical (surfactant free), Hexagonal disc } \\
\text { (SDS presence), Hexagonal bilayer disk } \\
\text { (PVP), Flower-like (PEG-10000); Particle } \\
\text { size: } 80-150 \mathrm{~nm} \text { (surfactant free), } \\
300 \& 200 \mathrm{~nm} \text { for length \& thickness (SDS), } \\
6 \mu \mathrm{m} \text { and } 4 \mu \mathrm{m} \text { for length \& thickness (PVP), } \\
\sim 200 \mathrm{~nm} \text { as average diameter (PEG-10000); } \\
\text { Band gap: } 3.24 \mathrm{eV} \text { (surfactant free), } 3.20 \mathrm{eV} \\
\text { (SDS), } 3.15 \mathrm{eV} \text { (PVP), and } 3.24 \mathrm{eV} \text { (PEG- } \\
\text { 10000) }\end{array}$ & $\begin{array}{l}\text { Photocatalyst in } \\
\text { the degradation of } \\
\text { Rhodamine-B } \\
\text { (Rh-B). }\end{array}$ & [122] \\
\hline $\begin{array}{l}\text { Microwave-assisted } \\
\text { without heat } \\
\text { treatment }\end{array}$ & $\begin{array}{l}\mathrm{Zn}(\mathrm{Ac})_{2} \cdot 2 \mathrm{H}_{2} \mathrm{O}, \text { ethylene } \\
\text { glycol, } \mathrm{H}_{2} \mathrm{O}(1.5 \text { wt. } \%)\end{array}$ & $\begin{array}{l}\text { Reaction temp: } 70^{\circ} \mathrm{C} \text {; Microwave } \\
\text { radiation: } 1,2, \& 3 \mathrm{~kW}, 2.45 \\
\mathrm{GHz}, 12 \text { min, } 4 \text { bar; Drying: } \\
\text { freeze drying }\end{array}$ & $\begin{array}{l}\text { Spherical shape, hexagonal shape; Particle } \\
\text { size: } 25-50 \mathrm{~nm} \text {; Crystallite size: } 23-48 \mathrm{~nm} \text {; } \\
\text { Surface area: } 40.1-40.6 \mathrm{~m}^{2} / \mathrm{g}\end{array}$ & & [123] \\
\hline $\begin{array}{l}\text { Solvothermal } \\
\text { synthesis with } \\
\text { conventional } \\
\text { heating }\end{array}$ & $\begin{array}{l}\mathrm{Zn}(\mathrm{Ac})_{2} \cdot 2 \mathrm{H}_{2} \mathrm{O}, \text { ethanol, } \\
\mathrm{KOH}\end{array}$ & $\begin{array}{l}\text { Reaction temp: } 60^{\circ} \mathrm{C} \text { for } 3 \mathrm{~h} \text {; } \\
\text { Drying temp: room temp. }\end{array}$ & $\begin{array}{l}\text { Spherical; Crystallite size: } 10.08 \mathrm{~nm} \text {; Particle } \\
\text { size (TEM/BET): 7.4/9.7 nm; Surface area: } \\
101.32 \mathrm{~m}^{2} / \mathrm{g}\end{array}$ & & [124] \\
\hline $\begin{array}{l}\text { Solvothermal } \\
\text { synthesis with } \\
\text { conventional } \\
\text { heating }\end{array}$ & $\begin{array}{l}\mathrm{Zn}(\mathrm{Ac})_{2} \cdot 2 \mathrm{H}_{2} \mathrm{O}, \text { ethanol, } \\
\mathrm{NaOH}, \mathrm{CTAB}, \text { wood } \\
\text { samples, FAS-17 }\end{array}$ & $\begin{array}{l}\text { Reaction temp: } 2 \mathrm{~h} \text { at room } \\
\text { temperature; Autoclave temp: } 90 \\
{ }^{\circ} \mathrm{C} \text { for } 4 \mathrm{~h} \text {; Drying temp: } 45^{\circ} \mathrm{C} \\
\text { for } 24 \mathrm{~h} .\end{array}$ & $\begin{array}{l}\text { Rod-like; Particle size: } 85 \mathrm{~nm} \& 1.5 \mu \mathrm{m} \\
\text { (diameter \& length) }\end{array}$ & UV resistor & [125] \\
\hline $\begin{array}{l}\text { Microwave-assisted } \\
\text { without heat } \\
\text { treatment }\end{array}$ & $\begin{array}{l}\mathrm{Zn}\left(\mathrm{NO}_{3}\right)_{2} \cdot 6 \mathrm{H}_{2} \mathrm{O}, \text { urea }(1: 5 \\
\text { molar ratio), }\end{array}$ & $\begin{array}{l}\text { Microwave irradiation: } 150{ }^{\circ} \mathrm{C} \text { for } \\
15 \text { min; Drying temp: } 50^{\circ} \mathrm{C} \\
\text { overnight }\end{array}$ & $\begin{array}{l}\text { Flower-like; Particle size (breadth): } 10 \mathrm{~nm} \text { to } \\
\text { micron }\end{array}$ & & [126] \\
\hline $\begin{array}{l}\text { Microwave-assisted } \\
\text { without heat } \\
\text { treatment }\end{array}$ & $\begin{array}{l}\mathrm{Zn}(\mathrm{Ac})_{2} \cdot 2 \mathrm{H}_{2} \mathrm{O}(1,2,4 \& 8 \\
\mathrm{mM}), \text { diethylene glycol, } \\
\text { oleic acid }\end{array}$ & $\begin{array}{l}\text { Microwave power: } 250{ }^{\circ} \mathrm{C} \text { for } 15 \\
\text { min; Drying temp: } 80^{\circ} \mathrm{C}\end{array}$ & Spherical; Particle size: 4-14 nm & & [127] \\
\hline $\begin{array}{l}\text { Microwave-assisted } \\
\text { with heat treatment }\end{array}$ & $\begin{array}{l}\mathrm{Zn}(\mathrm{Ac})_{2} \cdot 2 \mathrm{H}_{2} \mathrm{O} \text {, Isopropanol, } \\
\text { Diethanolamine }\end{array}$ & $\begin{array}{l}\text { Microwave power: } 150,175 \& \\
200{ }^{\circ} \mathrm{C} \text {; Calcination temp: } 100{ }^{\circ} \mathrm{C} \\
\text { for } 2 \mathrm{~h}\end{array}$ & Energy band gap: 3.38-3.94 eV & Photodetector & [128] \\
\hline $\begin{array}{l}\text { Microwave-assisted } \\
\text { with heat treatment }\end{array}$ & $\begin{array}{l}\mathrm{Zn}(\mathrm{Ac})_{2} \cdot 2 \mathrm{H}_{2} \mathrm{O}, \mathrm{NaOH} \\
\text { Dimethylformamide }\end{array}$ & $\begin{array}{l}\text { Microwave power: } 300 \mathrm{~W}, 1 \mathrm{~h} \text {; } \\
\text { Drying temp: } 60^{\circ} \mathrm{C} \text { for } 4 \mathrm{~h} \text {; } \\
\text { Calcination temp: } 500^{\circ} \mathrm{C}\end{array}$ & $\begin{array}{l}\text { Spherical; Crystallite size: } 24 \mathrm{~nm} \text { and } 26 \mathrm{~nm} \\
\text { (calcined sample); Energy band gap: } 3.26 \mathrm{eV} \\
\text { and } 3.20 \mathrm{eV} \text { (calcined sample) }\end{array}$ & $\begin{array}{l}\text { Dye (methyl } \\
\text { orange) removal } \\
\text { agent }\end{array}$ & [129] \\
\hline
\end{tabular}




\begin{tabular}{|c|c|c|c|c|c|}
\hline $\begin{array}{l}\text { Method of } \\
\text { synthesis }\end{array}$ & Precursor/Reactant & Synthesis condition & Properties & Applications & Reference \\
\hline $\begin{array}{l}\text { Microwave-assisted } \\
\text { without heat } \\
\text { treatment }\end{array}$ & $\begin{array}{l}\mathrm{Zn}(\mathrm{Ac})_{2} \cdot 2 \mathrm{H}_{2} \mathrm{O}, \mathrm{NaOH}, \\
\text { Triton } \mathrm{X}-100, \mathrm{H}_{2} \mathrm{O} / 2- \\
\text { ethoxyethanol (ET) / } \\
\text { ethylene glycol (EG). }\end{array}$ & $\begin{array}{l}\text { Microwave power: } 300 \mathrm{~W} \text { for } 3 \\
\text { min; Drying temp: room temp for } \\
72 \mathrm{~h}\end{array}$ & $\begin{array}{l}\text { Rod-like }\left(\mathrm{H}_{2} \mathrm{O} \& \mathrm{ET}\right) \text {, Flower-like }(\mathrm{EG}) ; \\
\text { Crystallite size: } 52.64 \mathrm{~nm}\left(\mathrm{H}_{2} \mathrm{O}\right), 63.70 \mathrm{~nm} \\
\text { (ET), } 24.02 \mathrm{~nm}(\mathrm{EG}) ; \text { Energy band gap: } 3.35 \\
\text { eV (ET), } 3.38 \mathrm{eV}\left(\mathrm{H}_{2} \mathrm{O}\right), 3.42 \mathrm{eV} \text { (EG) }\end{array}$ & $\begin{array}{l}\text { Photocatalyst in } \\
\text { the degradation of } \\
\text { methylene blue }\end{array}$ & [130] \\
\hline $\begin{array}{l}\text { Microwave-assisted } \\
\text { without heat } \\
\text { treatment }\end{array}$ & $\begin{array}{l}\mathrm{ZnCl}_{2}, \mathrm{C}_{18} \mathrm{H}_{33} \mathrm{NaO}_{2} \\
\mathrm{C}_{16} \mathrm{H}_{37} \mathrm{NO}, \mathrm{CH}_{3} \mathrm{OH} \\
\mathrm{C}_{36} \mathrm{H}_{66} \mathrm{O}_{4} \mathrm{Zn}, \mathrm{C}_{4} \mathrm{H}_{8} \mathrm{O}\end{array}$ & $\begin{array}{l}\text { Microwave irradiation: } 125,150 \\
175 \& 200^{\circ} \mathrm{C} \text { for } 5 \mathrm{~min}\end{array}$ & $\begin{array}{l}\text { Spherical; Particle size (radius/width): } 2.6 / 0.2 \\
\mathrm{~nm}\left(125^{\circ} \mathrm{C}\right), 2.7 / 0.3 \mathrm{~nm}\left(150^{\circ} \mathrm{C}\right), 3.1 / 0.3 \mathrm{~nm} \\
\left(175^{\circ} \mathrm{C}\right), 3.8 / 0.5 \mathrm{~nm}\left(200^{\circ} \mathrm{C}\right) \text {; Energy band } \\
\text { gap: }): 3.44 \mathrm{eV}\left(125^{\circ} \mathrm{C}\right), 3.40 \mathrm{eV}\left(150^{\circ} \mathrm{C}\right), \\
3.38 \mathrm{eV}\left(175^{\circ} \mathrm{C}\right), 3.36 \mathrm{eV}\left(200^{\circ} \mathrm{C}\right) ; \\
\text { Crystallite size: }): 4.55 \mathrm{~nm}\left(125^{\circ} \mathrm{C}\right), 5.88 \mathrm{~nm} \\
\left(150^{\circ} \mathrm{C}\right), 6.84 \mathrm{~nm}\left(175^{\circ} \mathrm{C}\right), 8.00 \mathrm{~nm}\left(200^{\circ} \mathrm{C}\right)\end{array}$ & & [131] \\
\hline $\begin{array}{l}\text { Microwave-assisted } \\
\text { with and without } \\
\text { heat treatment }\end{array}$ & $\begin{array}{l}\mathrm{Zn}(\mathrm{Ac})_{2} \cdot 2 \mathrm{H}_{2} \mathrm{O}, \text { diethylene } \\
\text { glycol, oleic acid, Toulene, } \\
\text { Si substrate }\end{array}$ & $\begin{array}{l}\text { Microwave power: } 220 \& 250{ }^{\circ} \mathrm{C} \\
\text { for } 10 \& 15 \mathrm{~min} \text {; Drying temp: } 80 \\
{ }^{\circ} \mathrm{C} \text { for } 30 \mathrm{~min} \text {; Drying temp of } \\
\text { substrate: } 100{ }^{\circ} \mathrm{C} \text { for } 3 \mathrm{~h} \text {; } \\
\text { Calcination temp of substrate: } \\
400{ }^{\circ} \mathrm{C} \text { for } 3 \mathrm{~h}\end{array}$ & Particle size : $5-12 \mathrm{~nm}$ & $\begin{array}{l}\text { Optoelectronic } \\
\text { devices }\end{array}$ & [132] \\
\hline
\end{tabular}

Table 3. Characteristics of $\mathrm{ZnO}$ nanostructures synthesized via different sol-gel routes.

\begin{tabular}{|c|c|c|c|c|c|}
\hline Method of synthesis & Precursor/Reactant & Synthesis condition & Properties & Applications & Reference \\
\hline $\begin{array}{l}\text { Sol-gel without heat } \\
\text { treatment }\end{array}$ & $\begin{array}{l}\mathrm{Zn}(\mathrm{Ac})_{2} \cdot 2 \mathrm{H}_{2} \mathrm{O}, \mathrm{CH}_{3} \mathrm{OH} \\
\mathrm{NaOH}\end{array}$ & $\begin{array}{l}\text { Sonication power: } 750 \mathrm{~W} \text { for } 30 \\
\text { min; Drying temp: } 80^{\circ} \mathrm{C} ; \mathrm{pH}=5 \text { - } \\
12\end{array}$ & $\begin{array}{l}\text { Particle size: } 1.3 \mathrm{~nm}(\mathrm{pH}, 7) \text { and } 73.8 \mathrm{~nm} \\
(\mathrm{pH}, 12) \\
\text { Crystallite size: } 10.94(\mathrm{pH}, 7), 17.44(\mathrm{pH}, \\
8), 38.27(\mathrm{pH}, 10), 74.04(\mathrm{pH}, 12)\end{array}$ & & [133] \\
\hline $\begin{array}{l}\text { Sol-gel without heat } \\
\text { treatment }\end{array}$ & $\mathrm{ZnCl}_{2}, \mathrm{NaOH}$ & $\begin{array}{l}\text { Reaction temp: } 50-90{ }^{\circ} \mathrm{C} \text {; Dripping } \\
\text { time: } 20-60 \mathrm{~min} \text {; Drying temp: } 70 \\
{ }^{\circ} \mathrm{C}\end{array}$ & Crystallite size: $21-37 \mathrm{~nm}$ & $\begin{array}{l}\text { Photocatalyst in } \\
\text { the degradation of } \\
\text { dyes }\end{array}$ & [134] \\
\hline $\begin{array}{l}\text { Sol-gel with heat } \\
\text { treatment }\end{array}$ & $\begin{array}{l}\mathrm{ZnSO}_{4} \cdot 7 \mathrm{H}_{2} \mathrm{O}, \mathrm{NaOH}(1: 1, \\
1: 2, \& 1: 3 \text { mole ratio })\end{array}$ & $\begin{array}{l}\text { Reaction time: } 8,10 \& 12 \mathrm{~h} \text {; Drying } \\
\text { temp: } 100^{\circ} \mathrm{C} \text {; Calcination temp: } \\
300,500, \& 700{ }^{\circ} \mathrm{C} \text { for } 2 \mathrm{~h}\end{array}$ & $\begin{array}{l}\text { Nanoplatelets }\left(1: 1,10 \mathrm{~h}, 300^{\circ} \mathrm{C}\right) \text {, } \\
\text { nanospheres }\left(1: 2,8 \mathrm{~h}, 700{ }^{\circ} \mathrm{C}\right) \text {, } \\
\text { nanoplatelets and nanorod }(1: 3,12 \mathrm{~h}, 500 \\
\left.{ }^{\circ} \mathrm{C}\right) \text {; Particle size: } 164-197 \mathrm{~nm}\left(300^{\circ} \mathrm{C}\right) \text {, } \\
1.73-167 \mathrm{~nm}\left(500{ }^{\circ} \mathrm{C}\right), 85-157 \mathrm{~nm}(700 \\
\left.{ }^{\circ} \mathrm{C}\right) \text {; Crystallite size: } 40.57 \mathrm{~nm}\end{array}$ & $\begin{array}{l}\text { Reinforcement } \\
\text { agent for } \\
\text { polymers }\end{array}$ & [135] \\
\hline $\begin{array}{l}\text { Sol-gel with heat } \\
\text { treatment }\end{array}$ & $\mathrm{Zn}\left(\mathrm{NO}_{3}\right)_{2} \cdot 6 \mathrm{H}_{2} \mathrm{O}, \mathrm{PVA}$ & $\begin{array}{l}\text { Reaction temp: } 80^{\circ} \mathrm{C} \text { for } 60 \mathrm{~h} \text {; } \\
\text { Drying temp: } 100^{\circ} \mathrm{C} \text { for } 24 \mathrm{~h} \text {; } \\
\text { Calcination temp: } 400-700{ }^{\circ} \mathrm{C}\end{array}$ & $\begin{array}{l}\text { Spherical; Crystallite size: } 15-51 \mathrm{~nm} \\
\left(400-550{ }^{\circ} \mathrm{C}\right) ; \text { Particle size }(\mathrm{TEM}): 15 \mathrm{~nm} \\
\left(400{ }^{\circ} \mathrm{C}\right), 25 \mathrm{~nm}\left(500^{\circ} \mathrm{C}\right)\end{array}$ & $\begin{array}{l}\text { Photocatalyst in } \\
\text { the degradation of } \\
\text { phenol }\end{array}$ & [136] \\
\hline
\end{tabular}




\begin{tabular}{|c|c|c|c|c|c|c|}
\hline $\begin{array}{l}\text { Method of } \\
\text { synthesis }\end{array}$ & Composite & Precursor/Reactant & Synthesis condition & Properties & Applications & Reference \\
\hline Solvothermal & $\begin{array}{l}\mathrm{Ag}-\mathrm{ZnO} \& \mathrm{Pt}- \\
\mathrm{ZnO}\end{array}$ & $\begin{array}{l}\mathrm{Zn}\left(\mathrm{NO}_{3}\right)_{2} \cdot 6 \mathrm{H}_{2} \mathrm{O}, \mathrm{AgNO}_{3}, \\
\mathrm{Pt}\left(\mathrm{C}_{5} \mathrm{H}_{7} \mathrm{O}_{2}\right)_{2}, \mathrm{CTAB}\end{array}$ & $\begin{array}{l}\text { Reaction temp: room temp for } 1 \\
\text { h; Autoclave temp: } 120^{\circ} \mathrm{C} 3 \& 6 \\
\text { h; Drying temp: } 80^{\circ} \mathrm{C}\end{array}$ & $\begin{array}{l}\text { Nanowires; Crystallite size: } 23-15.9 \\
\mathrm{~nm}(\mathrm{ZnO}), 193.6-63.6 \mathrm{~nm}(\mathrm{Pt}-\mathrm{ZnO}) \text {, } \\
\text { 12.9-11.5 nm (Ag-ZnO); Particle } \\
\text { size (TEM): 67-138 nm (ZnO), 133- } \\
143 \mathrm{~nm}(\mathrm{Ag}-\mathrm{ZnO}), 101-194 \mathrm{~nm} \text { (Pt- } \\
\mathrm{ZnO}) \\
\text { Surface area: } 5-33 \mathrm{~m}^{2} / \mathrm{g}(\mathrm{ZnO}), 23- \\
43 \mathrm{~m}^{2} / \mathrm{g}(\mathrm{Ag}-\mathrm{ZnO}), 4-15 \mathrm{~m}^{2} / \mathrm{g}(\mathrm{Pt}- \\
\mathrm{ZnO}) \text {; Energy band gap: } 3.21-3.24 \\
\mathrm{eV}(\mathrm{ZnO}), 3.23-3.29 \mathrm{eV}(\mathrm{Ag}-\mathrm{ZnO}) \text {, } \\
\text { 3.23-3.24 eV (Pt-ZnO) }\end{array}$ & $\begin{array}{l}\text { Photocatalyst in the } \\
\text { degradation of dyes }\end{array}$ & [137] \\
\hline Hydrothermal & $\mathrm{Ce}-\mathrm{ZnO}$ & $\begin{array}{l}\mathrm{ZnO}, \mathrm{CeO}_{2}, \mathrm{NaOH} / \mathrm{KOH}, \mathrm{n}- \\
\text { butyl amine }\end{array}$ & Autoclave temp: $100^{\circ} \mathrm{C}$ for $12 \mathrm{~h}$ & Hexagonal & $\begin{array}{l}\text { Photocatalyst in the } \\
\text { degradation of } \\
\text { synthetic wastewater }\end{array}$ & [138] \\
\hline Hydrothermal & $\mathrm{ZnO}-\mathrm{CdS}-\mathrm{Ag}$ & $\begin{array}{l}\mathrm{Zn}\left(\mathrm{NO}_{3}\right)_{2} \cdot 6 \mathrm{H}_{2} \mathrm{O}, \mathrm{C}_{2} \mathrm{H}_{4} \mathrm{O}_{3}, \\
\mathrm{KOH} \\
\mathrm{Cd}\left(\mathrm{NO}_{3}\right)_{2} \cdot 4 \mathrm{H}_{2} \mathrm{O}, \mathrm{C}_{2} \mathrm{H}_{5} \mathrm{NS} \\
\mathrm{AgNO}_{3}, \mathrm{~N}_{2} \mathrm{H}_{4}\end{array}$ & $\begin{array}{l}\text { Reaction temp }(\mathrm{ZnO}): 60{ }^{\circ} \mathrm{C} \text { for } 2 \\
\mathrm{~h} \text {; Autoclave temp }(\mathrm{ZnO}): 120{ }^{\circ} \mathrm{C} \\
\text { for } 48 \mathrm{~h} \text {; Drying temp }(\mathrm{ZnO}): 120 \\
{ }^{\circ} \mathrm{C} \text { for } 8 \mathrm{~h} \text {; Reaction time }(\mathrm{ZnO}- \\
\mathrm{CdS}): 1 \mathrm{~h} \text {; Drying temp }(\mathrm{ZnO}- \\
\mathrm{CdS}): 70^{\circ} \mathrm{C} \text {; Reaction time }(\mathrm{ZnO}- \\
\mathrm{CdS}-\mathrm{Ag}): 1 \mathrm{~h} \text {; Drying temp: } 70 \\
{ }^{\circ} \mathrm{C}\end{array}$ & $\begin{array}{l}\text { Rod-like; Crystallite size: } 23 \& 15 \\
\mathrm{~nm}(\mathrm{ZnO} \& \mathrm{CdS}) \text {; Surface area: } \\
18.9 \mathrm{~m}^{2} / \mathrm{g}(\mathrm{ZnO}-\mathrm{CdS}-\mathrm{Ag})\end{array}$ & $\begin{array}{l}\text { Photocatalyst against } \\
\text { E. coli }\end{array}$ & [139] \\
\hline Hydrothermal & $\begin{array}{l}\mathrm{ZnO}-\mathrm{CdS}(25: 75 \\
50: 50 \text { and } 75: 25)\end{array}$ & $\begin{array}{l}\mathrm{Zn}\left(\mathrm{NO}_{3}\right)_{2} \cdot 6 \mathrm{H}_{2} \mathrm{O}, \mathrm{ZnCl}_{2} \text {, } \\
\text { Thiourea }\end{array}$ & $\begin{array}{l}\text { Reaction time }(\mathrm{ZnO}): 1 \mathrm{~h} ; \mathrm{pH}= \\
12 \text {; Autoclave temp }(\mathrm{ZnO}): 150 \\
{ }^{\circ} \mathrm{C} \text { for } 3 \mathrm{~h} \text {; Autoclave temp } \\
(\mathrm{CdS}): 160{ }^{\circ} \mathrm{C} \text { for } 12 \mathrm{~h} \text {; Drying } \\
\text { temp }(\mathrm{CdS}): 100{ }^{\circ} \mathrm{C} \text {; Calcination } \\
\text { temp }(\mathrm{ZnO}-\mathrm{CdS}): 500{ }^{\circ} \mathrm{C} \text { for } 3 \mathrm{~h}\end{array}$ & $\begin{array}{l}\text { Flake-like (ZnO-CdS), Hexagonal } \\
(\mathrm{ZnO}) \text {, cubic }(\mathrm{CdS}) \text {; Crystallite size: } \\
\text { 13-33 nm (25:75), 17-35 nm } \\
\text { (50:50), } 21-38 \mathrm{~nm}(75: 25) ; \text { Energy } \\
\text { band gap: } 3.71 \mathrm{eV}(25: 75), 3.49 \mathrm{eV} \\
(50: 50), 3.35 \mathrm{eV}(75: 25)\end{array}$ & & [140] \\
\hline $\begin{array}{l}\text { Microwave- } \\
\text { assisted } \\
\text { hydrothermal }\end{array}$ & $\begin{array}{l}\mathrm{ZnO} \text {-Graphene } \\
\text { oxide }(\mathrm{GO})\end{array}$ & $\begin{array}{l}\mathrm{Zn}\left(\mathrm{NO}_{3}\right)_{2} \cdot 6 \mathrm{H}_{2} \mathrm{O}, \mathrm{PVP}, \\
\mathrm{HMTA}\end{array}$ & $\begin{array}{l}\text { Microwave power }(\mathrm{ZnO} / \mathrm{ZnO}- \\
\text { GO): } 300 \mathrm{~W}, 100{ }^{\circ} \mathrm{C} \text { for } 1 \mathrm{~h} / 45 \\
\text { min; Drying temp }(\mathrm{ZnO} / \mathrm{ZnO}- \\
\text { GO): } 85^{\circ} \mathrm{C}\end{array}$ & $\begin{array}{l}\text { Star-like (ZnO), flake-like sheets } \\
(\mathrm{GO}) \text {; Particle size: } 550 \mathrm{~nm}(\mathrm{ZnO}) \\
\text { Surface area: } 34.3 \mathrm{~m}^{2} / \mathrm{g}(\mathrm{ZnO}-\mathrm{GO})\end{array}$ & Electrocatalyst & [141] \\
\hline
\end{tabular}




\begin{tabular}{|c|c|c|c|c|c|c|}
\hline $\begin{array}{l}\text { Method of } \\
\text { synthesis }\end{array}$ & Composite & Precursor/Reactant & Synthesis condition & Properties & Applications & Reference \\
\hline $\begin{array}{l}\text { Microwave- } \\
\text { assisted } \\
\text { hydrothermal }\end{array}$ & $\mathrm{Ag}-\mathrm{ZnO}$ & $\begin{array}{l}\text { Carbinol, } \mathrm{Zn}(\mathrm{Ac})_{2} .2 \mathrm{H}_{2} \mathrm{O}, \\
\mathrm{AgNO}_{3}, \mathrm{CTAB}, \mathrm{NaBH}_{4}\end{array}$ & $\begin{array}{l}\text { Microwave power }(\mathrm{ZnO}): 900 \mathrm{~W} \text {, } \\
\text { 5, } 10 \text { \& } 15 \mathrm{~min} \text {; Microwave } \\
\text { power (Ag-ZnO): } 900 \mathrm{~W}, 20 \mathrm{~min}\end{array}$ & $\begin{array}{l}\text { Spherical (ZnO-5 min), hexagonal } \\
\text { (ZnO-10 min), spike-like ( } \mathrm{ZnO}-15 \\
\text { min), spike-like (Ag-ZnO-20 min); } \\
\text { Particle size: } 400-450 \mathrm{~nm}(\mathrm{ZnO}) \text {; } \\
\text { Length \& thickness of Ag-ZnO was } \\
1 \mu \& 30-40 \mathrm{~nm} \text {; Energy band gap: } \\
3.72 \mathrm{eV}(\mathrm{Ag}-\mathrm{ZnO})\end{array}$ & $\begin{array}{l}\text { Sensor to } \\
\text { biomolecules }\end{array}$ & [142] \\
\hline $\begin{array}{l}\text { Microwave- } \\
\text { assisted } \\
\text { hydrothermal }\end{array}$ & & $\begin{array}{l}\mathrm{CTAB}, \mathrm{Zn}(\mathrm{Ac})_{2} .2 \mathrm{H}_{2} \mathrm{O} \\
\mathrm{AgNO}_{3}, \mathrm{Na}_{2} \mathrm{O}_{2} \text { mole ratio; } \\
\text { CTBA: } \mathrm{Zn}: \mathrm{Ag}=1: 1.5: 0.05)\end{array}$ & $\begin{array}{l}\text { Reaction time: } 30 \mathrm{~min} \text {, Autoclave } \\
\text { temp: } 200^{\circ} \mathrm{C} \text {, Microwave } \\
\text { irradiation: } 300 \mathrm{~W}, 200^{\circ} \mathrm{C} \text { for } 3 \text {, } \\
4.5 \& 6 \mathrm{~h} \text {, Drying temp: } 80{ }^{\circ} \mathrm{C} \text {, } \\
\text { Calcination temp: } 300^{\circ} \mathrm{C} \text { for } 2 \mathrm{~h}\end{array}$ & $\begin{array}{l}\text { Rod-like stacked into a } \\
\text { chrysanthemum-like (flower) } \\
\text { structure; Crystallite size: } 4.1,3.3 \text {, } \\
3.2 \& 3.4 \mathrm{~nm} \text { (ZnO, Ag-ZnO-3, } 4.5 \\
\& 6 \mathrm{~h}) \text {; Energy band gap: } 3.21,3.17 \text {, } \\
3.14,3.12 \mathrm{eV} \text { ( } \mathrm{ZnO}, \mathrm{Ag}-\mathrm{ZnO}-3,4.5 \\
\& 6 \mathrm{~h}) \text {; Surface area: } 1.05,6.71 \\
6.40,6.74 \mathrm{~m}^{2} / \mathrm{g}(\mathrm{ZnO}, \mathrm{Ag}-\mathrm{ZnO}-3 \\
4.5 \& 6 \mathrm{~h})\end{array}$ & $\begin{array}{l}\text { Photocatalyst in the } \\
\text { degradation of RhB }\end{array}$ & [143] \\
\hline Sol-gel & $\mathrm{Al}-\mathrm{ZnO}$ & $\begin{array}{l}\mathrm{Zn}(\mathrm{Ac})_{2} \cdot 2 \mathrm{H}_{2} \mathrm{O}, \mathrm{Al}\left(\mathrm{NO}_{3}\right)_{3}(0 \\
3,6,9 \& 12 \% \% \mathrm{wt})\end{array}$ & $\begin{array}{l}\text { Reaction temp: } 80^{\circ} \mathrm{C} \text { for } 1 \mathrm{~h} \text {; } \\
\text { Drying temp: } 100^{\circ} \mathrm{C} \text {; Calcination } \\
\text { temp: } 600{ }^{\circ} \mathrm{C} \text { for } 4 \mathrm{~h}\end{array}$ & $\begin{array}{l}\text { Rod-like }(\mathrm{ZnO}) \text {, spherical }(\mathrm{Al}-\mathrm{ZnO}) \\
\text { Crystallite size: } 78.8,49.6,29.9 \\
35.0 \text { \& } 52.4 \mathrm{~nm} \text { (Al-ZnO-0, 3, 6, } 9 \\
\& 12 \%)\end{array}$ & Sensor to $\mathrm{NH}_{3}$ gas & [144] \\
\hline $\begin{array}{l}\text { Microwave- } \\
\text { assisted } \\
\text { hydrothermal }\end{array}$ & $\mathrm{ZnO}-\mathrm{N}$ & $\begin{array}{l}\mathrm{Zn}(\mathrm{Ac})_{2} \cdot 2 \mathrm{H}_{2} \mathrm{O}, \mathrm{NaOH}, \text { urea } \\
(5 \%)\end{array}$ & $\begin{array}{l}\text { Reaction time }(\mathrm{ZnO} / \mathrm{ZnO}-\mathrm{N}): 10 \\
\text { min; Microwave power } \\
(\mathrm{ZnO} / \mathrm{ZnO}-\mathrm{N}): 800 \mathrm{~W}, 100^{\circ} \mathrm{C} \text { for } \\
1 \mathrm{~h}\end{array}$ & $\begin{array}{l}\text { Flower-like with array of nanorods } \\
\text { Particle size (Length): } 1.2-1.7 \mu \mathrm{m} \\
(\mathrm{ZnO}), 2.0-2.4 \mu \mathrm{m}(\mathrm{ZnO}-\mathrm{N})\end{array}$ & $\begin{array}{l}\text { Photocatalyst in the } \\
\text { degradation of Rh-B } \\
\& \mathrm{Cr}(\mathrm{VI})\end{array}$ & [145] \\
\hline $\begin{array}{l}\text { Microwave } \\
\text { solvothermal }\end{array}$ & Co-Mn-ZnO & $\begin{array}{l}\mathrm{Zn}(\mathrm{Ac})_{2} \cdot 2 \mathrm{H}_{2} \mathrm{O} \\
\mathrm{Mn}(\mathrm{Ac})_{2} \cdot 4 \mathrm{H}_{2} \mathrm{O} \\
\mathrm{Con}(\mathrm{Ac})_{2} \cdot 4 \mathrm{H}_{2} \mathrm{O}\end{array}$ & $\begin{array}{l}\text { Reaction temp: } 70^{\circ} \mathrm{C} ; \text { Microwave } \\
\text { power: } 100 \%, 190{ }^{\circ} \mathrm{C}, 25 \mathrm{~min} ; \\
\text { Drying temp: freeze drying }\end{array}$ & $\begin{array}{l}\text { Spherical; Particle size: } 20-40 \mathrm{~nm} \text {; } \\
\text { Surface area: } 45.8-56.4 \mathrm{~m}^{2} / \mathrm{g}(\mathrm{Co}- \\
\mathrm{Mn}-\mathrm{ZnO}), 39.8 \mathrm{~m}^{2} / \mathrm{g}(\mathrm{ZnO}) \\
\text { Crystallite size: } 19-22 \mathrm{~nm}(\mathrm{Co}-\mathrm{Mn}- \\
\mathrm{ZnO}), 22 \mathrm{~nm}(\mathrm{ZnO})\end{array}$ & & [146] \\
\hline Sol-gel & $\mathrm{Zr}-\mathrm{Cr} / \mathrm{ZnO}$ & $\begin{array}{l}\mathrm{Zn}\left(\mathrm{NO}_{3}\right)_{2} \cdot 6 \mathrm{H}_{2} \mathrm{O} \\
\mathrm{Cr}\left(\mathrm{NO}_{3}\right)_{3} \cdot 9 \mathrm{H}_{2} \mathrm{O}, \text { oxalic acid }\end{array}$ & $\begin{array}{l}\text { Aging conditions: } 2 \mathrm{~h} \text { at room } \\
\text { temperature, } 75^{\circ} \mathrm{C} \text { for } 2 \mathrm{~h} \text { and } \\
120^{\circ} \mathrm{C} \text { for another } 10 \mathrm{~h} \text { in an } \\
\text { oven; Calcination temp: } 400{ }^{\circ} \mathrm{C} \\
\text { for } 6 \mathrm{~h}\end{array}$ & $\begin{array}{l}\text { Hexagonal nanoplates and nano- } \\
\text { polyhedron shapes }\end{array}$ & Catalyst & [147] \\
\hline
\end{tabular}


https://doi.org/10.33263/BRIAC123.42614292

\begin{tabular}{|c|c|c|c|c|c|c|}
\hline $\begin{array}{l}\text { Method of } \\
\text { synthesis }\end{array}$ & Composite & Precursor/Reactant & Synthesis condition & Properties & Applications & Reference \\
\hline $\begin{array}{l}\text { Microwave- } \\
\text { assisted } \\
\text { hydrothermal }\end{array}$ & $\mathrm{Mn}-\mathrm{ZnO}$ & $\begin{array}{l}\mathrm{Mn}(\mathrm{Ac})_{2} \cdot 4 \mathrm{H}_{2} \mathrm{O}(1,5, \& 10 \\
\mathrm{wt} \%), \mathrm{Zn}(\mathrm{Ac})_{2} \cdot 2 \mathrm{H}_{2} \mathrm{O}\end{array}$ & $\begin{array}{l}\text { Reaction temp: } 30^{\circ} \mathrm{C} \text {; Microwave } \\
\text { power: } 300 \mathrm{~W}, 95^{\circ} \mathrm{C} \text { for } 10,20 \& \\
30 \mathrm{~min} \text {; Drying temp: } 60{ }^{\circ} \mathrm{C} \text { for } \\
24 \mathrm{~h} \text {; Calcination temp: } 500{ }^{\circ} \mathrm{C} \\
\text { for } 2 \mathrm{~h} \text {. }\end{array}$ & $\begin{array}{l}\text { Rod-like; Particle size (diameter): } \\
600-50 \mathrm{~nm}\end{array}$ & Sensors & [148] \\
\hline $\begin{array}{l}\text { Microwave } \\
\text { solvothermal } \\
\text { synthesis }\end{array}$ & & $\begin{array}{l}\mathrm{Zn}(\mathrm{Ac})_{2} \cdot 2 \mathrm{H}_{2} \mathrm{O} \\
\mathrm{Mn}(\mathrm{Ac})_{2} \cdot 4 \mathrm{H} 2 \mathrm{O}(1,5,10,15 \\
20,25 \mathrm{~mol} \% \mathrm{Mn})\end{array}$ & $\begin{array}{l}\text { Reaction temp: } 70^{\circ} \mathrm{C} \text {; Microwave } \\
\text { power: } 600 \mathrm{~W}, 2.45 \mathrm{GHz}, 200{ }^{\circ} \mathrm{C} \\
\text { for } 25 \text { min; Drying mode: freeze } \\
\text { drying }\end{array}$ & $\begin{array}{l}\text { Spherical shape, flower-like (5-25 } \\
\text { mol \%); Particle size: } 30-35 \mathrm{~nm} \text { to } \\
15-25 \mathrm{~nm}(0-20 \text { mol } \%), 20-25 \mathrm{~nm} \\
(25 \mathrm{~mol} \%) \text {; Crystallite size: } 22,21 \\
18,17,18,16, \& 19 \mathrm{~nm}(0,1,5,10 \\
15,20 \& 25 \mathrm{~mol} \%) ; \text { Specific area: } \\
28,30,24,20,21,17 \& 19 \mathrm{~m}^{2} / \mathrm{g}(0, \\
1,5,10,15,20 \& 25 \mathrm{~mol} \%)\end{array}$ & & [149] \\
\hline $\begin{array}{l}\text { Microwave } \\
\text { assisted method }\end{array}$ & $\mathrm{Co}-\mathrm{ZnO}$ & $\begin{array}{l}\mathrm{Zn}(\mathrm{Ac})_{2} \cdot 2 \mathrm{H}_{2} \mathrm{O}, \mathrm{C}_{4} \mathrm{H}_{6} \mathrm{CoO}_{4} \\
\text { Urea, Ethylene glycol }\end{array}$ & $\begin{array}{l}\text { Reaction time: } 1 \mathrm{~h} \text {; Microwave } \\
\text { temp: } 80^{\circ} \mathrm{C} \text {; Calcination temp: } \\
300^{\circ} \mathrm{C} \text { for } 1 \mathrm{~h}\end{array}$ & $\begin{array}{l}\text { Crystallite size } 18-28 \mathrm{~nm} \\
\text { Energy band gap: } 2.24-3.26 \mathrm{eV} \\
\text { Spherical }\end{array}$ & & [150] \\
\hline $\begin{array}{l}\text { Microwave } \\
\text { assisted method }\end{array}$ & & $\begin{array}{l}\mathrm{Zn}(\mathrm{Ac})_{2} .2 \mathrm{H}_{2} \mathrm{O}, \text { diethylene } \\
\text { glycol }(\mathrm{DEG}), \mathrm{Co}(\mathrm{II})(\mathrm{Acac})_{2}, \\
\text { oleic acid }\end{array}$ & $\begin{array}{l}\text { Microwave power: } 250{ }^{\circ} \mathrm{C} \text { for } 25 \\
\text { min; Calcination temp: } 400{ }^{\circ} \mathrm{C} \text {; } \\
\text { Drying temp: } 80^{\circ} \mathrm{C}\end{array}$ & $\begin{array}{l}\text { Hexagonal; Crystallite size: } 8.9-9.9 \\
\text { nm, 16-19 nm (calcined sample); } \\
\text { Particle size: } 15-35 \mathrm{~nm} \text {; Energy } \\
\text { band gap: } 2.79-3.23 \mathrm{eV}\end{array}$ & $\begin{array}{l}\text { Electroluminescent } \\
\text { material for polymer } \\
\text { light emitting } \\
\text { diodes }\end{array}$ & {$[132]$} \\
\hline $\begin{array}{l}\text { Microwave- } \\
\text { Assisted } \\
\text { Synthesis }\end{array}$ & $\begin{array}{l}\text { AL-ZnO, Ga- } \\
\mathrm{ZnO}, \mathrm{Al}-\mathrm{Ga}-\mathrm{ZnO}\end{array}$ & $\begin{array}{l}\mathrm{Zn}(\mathrm{Ac})_{2} \cdot 2 \mathrm{H}_{2} \mathrm{O}, \mathrm{AlCl}_{3} \cdot 6 \mathrm{H}_{2} \mathrm{O}, \\
\mathrm{Ga}\left(\mathrm{NO}_{3}\right)_{3} \cdot \mathrm{xH}_{2} \mathrm{O}, \text { diethylene } \\
\text { glycol }(\mathrm{DEG})\end{array}$ & $\begin{array}{l}\text { Reaction time: } 1 \mathrm{~h} \text {; Microwave } \\
\text { power: } 2.45 \mathrm{GHz}, 1500 \mathrm{~W}, 200 \\
{ }^{\circ} \mathrm{C} \text { for } 30 \mathrm{~min} \text {; Drying temp: } 60 \\
{ }^{\circ} \mathrm{C} \text { for } 1 \mathrm{~h} \text {; Calcination temp: } 450 \\
{ }^{\circ} \mathrm{C} \text { for } 1 \mathrm{~h}\end{array}$ & $\begin{array}{l}\text { Spherical; Particle size: } 100 \mathrm{~nm} \\
(\mathrm{Ga}-\mathrm{ZnO}), 250 \mathrm{~nm}(\mathrm{Al}-\mathrm{ZnO}), \sim 85 \\
\mathrm{~nm}(\mathrm{Al}-\mathrm{Ga}-\mathrm{ZnO})\end{array}$ & $\begin{array}{l}\text { Conducting material } \\
\text { for Transparent } \\
\text { conducting oxides } \\
\text { (TCO) coatings }\end{array}$ & [151] \\
\hline Reflux method & $\mathrm{Cu}-\mathrm{ZnO}$ & $\mathrm{Zn}\left(\mathrm{NO}_{3}\right)_{2} \cdot 6 \mathrm{H}_{2} \mathrm{O}, \mathrm{CuCl}_{2} \cdot 2 \mathrm{H}_{2} \mathrm{O}$ & $\begin{array}{l}\text { Reflux time }(\mathrm{ZnO}): 3 \mathrm{~h} \text {; Reflux } \\
\text { time }(\mathrm{Cu}-\mathrm{ZnO}): 3-9 \mathrm{~h} \text {; Drying } \\
\text { temp }(\mathrm{Cu}-\mathrm{ZnO}): 80^{\circ} \mathrm{C} \text {. }\end{array}$ & $\begin{array}{l}\text { Cube-like, maize corn seed-like, } \\
\text { rod-like }(\mathrm{ZnO}) \text {, maize corn seed-like } \\
(\mathrm{Cu}-\mathrm{ZnO}) \text {; Crystallite size: } 28 \mathrm{~nm} \\
(\mathrm{ZnO}), 20 \mathrm{~nm}(\mathrm{Cu}-\mathrm{ZnO}) \text {; Particle }\end{array}$ & $\begin{array}{l}\text { Photocatalyst in the } \\
\text { degradation of } \\
\text { methyl orange (MO) }\end{array}$ & [152] \\
\hline
\end{tabular}




\begin{tabular}{|c|c|c|c|c|c|c|}
\hline $\begin{array}{l}\text { Method of } \\
\text { synthesis }\end{array}$ & Composite & Precursor/Reactant & Synthesis condition & Properties & Applications & Reference \\
\hline & & & & $\begin{array}{l}\text { size (diameter \& length): } 30-35 \mathrm{~nm} \\
\& 90 \mathrm{~nm}(\mathrm{Cu}-\mathrm{ZnO}) \text {; Energy band } \\
\text { gap: } 3.38-3.46 \mathrm{eV}(\mathrm{Cu}-\mathrm{ZnO})\end{array}$ & & \\
\hline $\begin{array}{l}\text { Solvent-thermal } \\
\text { technique }\end{array}$ & $\mathrm{Fe}_{3} \mathrm{O}_{4} / \mathrm{ZnO}$ & $\begin{array}{l}\mathrm{C}_{6} \mathrm{H}_{5} \mathrm{Na}_{3} \mathrm{O}_{7} \cdot 2 \mathrm{H}_{2} \mathrm{O}, \mathrm{CH}_{3^{-}} \\
\mathrm{COONa}, \mathrm{FeCl}_{3} \cdot 6 \mathrm{H}_{2} \mathrm{O}, \\
\text { diethylene glycol (DEG), } \\
\text { ethylene glycol (EG), } \\
\text { tetraethyl orthosilicate } \\
\text { (TEOS) }\end{array}$ & $\begin{array}{l}\text { Reaction temp }(\mathrm{ZnO}): 40^{\circ} \mathrm{C} \text { for } 3 \\
\text { h; Drying temp ( } \mathrm{ZnO} \& \\
\text { composite): } 70^{\circ} \mathrm{C} \text { for } 3 \mathrm{~h} \text {; } \\
\text { Microwave temp (composite): } \\
160^{\circ} \mathrm{C} \text { for } 15,30,60 \mathrm{~min},\end{array}$ & $\begin{array}{l}\text { Spherical; Surface area: } 22.30 \mathrm{~m}^{2} / \mathrm{g} \\
\left.\text { (composite with } 10 \% \mathrm{Fe}_{3} \mathrm{O}_{4}\right), 27.52 \\
\mathrm{~m}^{2} / \mathrm{g}\left(\text { composite with } 20 \% \mathrm{Fe}_{3} \mathrm{O}_{4} \text { ), }\right. \\
20.10 \mathrm{~m}^{2} / \mathrm{g} \text { (composite with } 30 \% \\
\left.\mathrm{Fe}_{3} \mathrm{O}_{4}\right) ; \text { Energy band gap: } 3.21-3.24 \\
\mathrm{eV}\end{array}$ & $\begin{array}{l}\text { Photocatalyst in the } \\
\text { degradation of } 4- \\
\text { Nitrophenol (4-NP) }\end{array}$ & [153] \\
\hline Solvothermal & $\mathrm{CeO}_{2}-\mathrm{ZnO}$ & $\begin{array}{l}\mathrm{Zn}(\mathrm{Ac})_{2} \cdot 2 \mathrm{H}_{2} \mathrm{O}, \mathrm{CeCl}_{3} \cdot 7 \mathrm{H}_{2} \mathrm{O} \\
\mathrm{Na}_{3} \mathrm{C}_{6} \mathrm{H}_{5} \mathrm{O}_{7}\end{array}$ & $\begin{array}{l}\text { Autoclave temp: } 200^{\circ} \mathrm{C} \text { for } 10 \mathrm{~h} \text {; } \\
\text { Calcination temp: } 500^{\circ} \mathrm{C} \text { for } 2 \mathrm{~h}\end{array}$ & $\begin{array}{l}\text { Chain-like structure; Crystallite size: } \\
9.5 \mathrm{~nm} \text {; Surface area: } 40.9 \mathrm{~m}^{2} / \mathrm{g} \text {, }\end{array}$ & $\begin{array}{l}\text { Gas sensor and in } \\
\text { diagnosis of diabetes } \\
\text { and chemical } \\
\text { detection }\end{array}$ & [154] \\
\hline $\begin{array}{l}\text { Microwave } \\
\text { method }\end{array}$ & $\mathrm{Fe}-\mathrm{ZnO}$ & $\begin{array}{l}\mathrm{Zn}(\mathrm{OAc})_{2} \cdot 2 \mathrm{H}_{2} \mathrm{O}, \mathrm{NaOH} \\
\mathrm{Fe}\left(\mathrm{NO}_{3}\right)_{3} \cdot 9 \mathrm{H}_{2} \mathrm{O}\end{array}$ & $\begin{array}{l}\text { Microwave power: } 140 \mathrm{~W} \text { for } 2 \\
\min (\mathrm{ZnO}) \text {; Drying temp: } 200{ }^{\circ} \mathrm{C} \\
\text { for } 1 \mathrm{~h}(\mathrm{ZnO}) \text {; Drying temp: } 120 \\
{ }^{\circ} \mathrm{C} \text { for } 4-5 \mathrm{~min}(\mathrm{Fe}-\mathrm{ZnO}) ; \\
\text { Calcination temp: } 300{ }^{\circ} \mathrm{C}\end{array}$ & $\begin{array}{l}\text { Energy band gap: } 3.20-3.24 \mathrm{eV} ; \\
\text { Particle size (diameter): } 17.4-11.29 \\
\mathrm{~nm}(\mathrm{Fe}-\mathrm{ZnO})\end{array}$ & $\begin{array}{l}\text { Anti-reflector } \\
\text { coating }\end{array}$ & [155] \\
\hline $\begin{array}{l}\text { Microwave- } \\
\text { assisted } \\
\text { hydrothermal }\end{array}$ & & $\begin{array}{l}\mathrm{Zn}\left(\mathrm{NO}_{3}\right)_{2} \cdot 6 \mathrm{H}_{2} \mathrm{O} \\
\mathrm{Fe}\left(\mathrm{NO}_{3}\right)_{3} \cdot 9 \mathrm{H}_{2} \mathrm{O}(0,1,3,5,7 \\
\& 10 \% \text { mole })\end{array}$ & $\begin{array}{l}\text { Microwave power: } 700 \mathrm{~W}, 5.8- \\
6.2 \mathrm{MPa}, 20 \mathrm{~min} \text {; Drying temp: } \\
60{ }^{\circ} \mathrm{C} \text { by } 18 \mathrm{~h} ; \mathrm{pH}=9.5\end{array}$ & $\begin{array}{l}\text { Rod-like, hexagonal prism; } \\
\text { Crystallite size: } 130 \mathrm{~nm}(5 \% \mathrm{Fe}), 60 \\
\mathrm{~nm}(7 \% \mathrm{Fe}), 30 \mathrm{~nm}(10 \% \mathrm{Fe}), 60 \\
\mathrm{~nm}(<5 \% \mathrm{Fe}) \text {; Particle size: } 392- \\
296 \mathrm{~nm}(0-10 \% \mathrm{Fe})\end{array}$ & $\begin{array}{l}\text { Useful in biomedical } \\
\text { applications }\end{array}$ & [156] \\
\hline $\begin{array}{l}\text { Hydrothermal } \\
\text { method }\end{array}$ & Zn-Al-Gd & $\begin{array}{l}\mathrm{Zn}(\mathrm{Ac})_{2} \cdot 2 \mathrm{H}_{2} \mathrm{O} \\
\mathrm{Al}\left(\mathrm{NO}_{3}\right)_{3} \cdot 2 \mathrm{H}_{2} \mathrm{O} \\
\mathrm{Gd}(\mathrm{NO})_{3} \cdot 6 \mathrm{H}_{2} \mathrm{O}, \mathrm{NH}_{4} \mathrm{OH}\end{array}$ & $\begin{array}{l}\text { Reaction time: } 3 \mathrm{~h} \text {; Autoclave } \\
\text { temp: } 160{ }^{\circ} \mathrm{C} \text { for } 24 \mathrm{~h} \text {; calcination } \\
\text { temp: } 600{ }^{\circ} \mathrm{C} ; \mathrm{pH}=\sim 9\end{array}$ & $\begin{array}{l}\text { Mixture of urchin-like and rod-like } \\
\text { structures; Crystallite size: } 67-47 \\
\mathrm{~nm}\end{array}$ & & [157] \\
\hline $\begin{array}{l}\text { Hydrothermal } \\
\text { method }\end{array}$ & $\mathrm{Eu}-\mathrm{ZnO}$ & $\begin{array}{l}\mathrm{Zn}(\mathrm{Ac})_{2} \cdot 2 \mathrm{H}_{2} \mathrm{O}, \\
\mathrm{Eu}\left(\mathrm{CH}_{3} \mathrm{CO}_{2}\right)_{3} \cdot \mathrm{xH} 2 \mathrm{O}(1,3 \& \\
5 \mathrm{wt} \%)\end{array}$ & $\begin{array}{l}\text { Reaction time: } 20 \mathrm{~min} \text {; Autoclave } \\
\text { temp: } 150^{\circ} \mathrm{C} \text { for } 8 \mathrm{~h} \text {; Drying } \\
\text { temp: } 80^{\circ} \mathrm{C} \text { for } 24 \mathrm{~h}, \mathrm{pH}=10\end{array}$ & $\begin{array}{l}\text { Spherical (undoped \& doped); } \\
\text { Crystallite size: } 26 \mathrm{~nm} \text { (undoped), } \\
24,15, \& 18 \mathrm{~nm}(1 \%, 3 \% \& 5 \% \\
\text { Eu); Energy band gap: } 3.18 \mathrm{eV} \\
\text { (undoped), } 3.05,3.00, \& 2.94 \mathrm{eV} \\
(1 \%, 3 \% \& 5 \% \mathrm{Eu})\end{array}$ & $\begin{array}{l}\text { Optoelectronic } \\
\text { devices }\end{array}$ & [158] \\
\hline $\begin{array}{l}\text { Single-step } \\
\text { aerosol process }\end{array}$ & ZnO-Carbon dots & $\mathrm{Zn}\left(\mathrm{NO}_{3}\right)_{2} \cdot 6 \mathrm{H}_{2} \mathrm{O}, \mathrm{C}_{6} \mathrm{H}_{8} \mathrm{O}_{7}$ & $\begin{array}{l}\text { Reaction time: } 1 \mathrm{~h} \text {; Heating temp: } \\
\text { 500, 550, } 600 \& 650^{\circ} \mathrm{C} \text {; } \\
\text { Annealing temp: } 600^{\circ} \mathrm{C} \text { for } 3 \mathrm{~h}\end{array}$ & Spherical & $\begin{array}{l}\text { Photocatalyst for } \\
\mathrm{CO}_{2} \text { reduction }\end{array}$ & [159] \\
\hline
\end{tabular}


https://doi.org/10.33263/BRIAC123.42614292

\begin{tabular}{l|l|l|l|l|l}
\hline $\begin{array}{c}\text { Method of } \\
\text { synthesis }\end{array}$ & \multicolumn{1}{|c|}{ Composite } & \multicolumn{1}{|c|}{ Precursor/Reactant } & \multicolumn{1}{c}{ Synthesis condition } & \multicolumn{1}{c}{ Properties } \\
\hline $\begin{array}{l}\text { Microwave- } \\
\text { assisted }\end{array}$ & $\mathrm{Al}_{2} \mathrm{O}_{3}-\mathrm{ZnO}$ & $\begin{array}{l}\text { Commercial } \mathrm{ZnO} \mathrm{NPs}, \\
\text { Aluminium triisopropoxide } \\
(0.5,1.0 \& 1.5 \mathrm{~g})\end{array}$ & $\begin{array}{l}\text { Grinding time: } 10 \mathrm{~min} ; \\
\text { Microwave temp: } 500 \mathrm{~W}, 2.45 \\
\mathrm{GHz}, 70{ }^{\circ} \mathrm{C} \text { for } 5 \mathrm{~min} \text {; Aging } \\
\text { time: } 3 \mathrm{~h} \text {; Drying temp: } 110^{\circ} \mathrm{C} \\
\text { for } 2 \mathrm{~h}\end{array}$ & $\begin{array}{l}\text { Particle size: } 20-30 \mathrm{~nm}(\mathrm{ZnO}), \\
\text { Photocatalyst in the } \\
\text { degradation of } \\
\text { methylene blue } \\
(\mathrm{MB})\end{array}$ \\
\hline
\end{tabular}




\section{Conclusions}

The multi-functionality of $\mathrm{ZnO}$ nanomaterials continues to grow as the developments of new methods to their synthesis are explored. These new methods allow for the control of the morphology, which subsequently affects their properties in producing innovative devices for its various applications. The improvement of microwave technology has made it easy to synthesize different desirable morphologies of nanomaterials with speed and purity. This technology is applied to different synthesis methods (solvothermal, hydrothermal, and sol-gel), it was possible to obtain desirable morphologies as the reaction mechanism could be controlled. Besides the heating mechanism, other factors such as the type of precursor, surfactants, alkaline source, annealing temperature, and dopants affect the morphological structure.

\section{Funding}

This research was funded by Universiti Malaysia Sarawak, Tun Openg Chair, with research grant code: F07/TOC/1738/2018.

\section{Acknowledgments}

The authors would like to thank the funding provided by Universiti Malaysia Sarawak, Tun Openg Chair, with research grant code: F07/TOC/1738/2018. We also acknowledge the contribution of colleagues from the Faculty of Resource Science and Technology (FRST), Geochemistry Laboratory, and Analytical Laboratory, Universiti Malaysia Sarawak.

\section{Conflict of Interest}

The authors declare no conflict of interest.

\section{References}

1. Jiang, J.; Pi, J.; Cai, J. The advancing of zinc oxide nanoparticles for biomedical applications. Bioinor. Chem. Appls 2018, 1-18, https://doi.org/10.1155/2018/1062562.

2. Chaudhary, S.; Umar, A.; Bhasin, K.K.; Baskoutas, S. Chemical sensing applications of ZnO nanomaterials. Mater. 2018, 11, 1-38, https://doi.org/10.3390/ma11020287.

3. Sarmah, K.; Pratihar, S. Synthesis, characterization and photocatalytic application of iron oxalate capped Fe, $\mathrm{Fe}-\mathrm{Cu}$, FeCo, and Fe-Mn oxide nanomaterial. ACS Sustain. Chem. Eng. 2017, 5, 310-324, https://doi.org/10.1021/acssuschemeng.6b01673.

4. Das, P.; Sarmah, K.; Hussain, N.; Pratihar, S.; Das, S.; Bhattacharyya, P.; Patil, S.A.; Kim, H.S.; Iqbal, M.; Khazie, A.; Bhattacharyya, S.S. Novel synthesis of an iron oxalate capped iron oxide nanomaterial; a unique soil conditioner and slow release eco-friendly source of iron sustenance in plants. RSC Adv. 2016, 6, 10301225, https://doi.org/10.1039/C6RA18840K.

5. Alshamsi, H.A.H.; Hussein, B.S. Hydrothermal preparation of silver doping zinc oxide nanoparticles: synthesis, characterization and photocatalytic activities. Orient. J. Chem. 2018, 34, 1898-1907, https://dx.doi.org/10.13005/ojc/3404025.

6. Jin, S-E.; Jin, H-E. Synthesis, characterization, and three-dimensional structure generation of zinc oxidebased nanomedicine for biomedical applications. Pharma. 2019, 11, 575, 1-26, https://doi.org/10.3390/pharmaceutics11110575.

7. Kwoka, M.; Lyson-Sypien, B.; Kulis, A.; Maslyk, M.; Borysiewicz, M.A.; Kaminska, E.; Szuber, J. Surface properties of nanostructured, porous $\mathrm{ZnO}$ thin films prepared by direct current reactive magnetron sputtering. Mater. 2018, 11, 131, https://doi.org/10.3390ma11010131. 
8. Ren, X.; Zi, W.;Wei, Q.; Liu, S. Fabrication gallium/graphene core-shell Nanoparticles by pulsed laser deposition and their applications in surface enhanced Raman scattering. Mater. Lett. 2015, 143, 194-196, https://doi.org/10.1016/j.matlet.2014.12.089.

9. Chhikara, D.; Senthil, K.M.; Srivatsa, K.M.K. On the synthesis of Zn/ZnO core-shell solid microspheres on quartz substrate by thermal evaporation technique. Superlatt. Microstruc. 2015, 82, 368-377, https://doi.org/10.1016/j.spmi.2015.02.036.

10. Varnamkhasti, M.G.; Fallah, H.R.; Zadsar, M. Effect of heat treatment on characteristics of nanocrystalline $\mathrm{ZnO}$ films by electron beam evaporation. Vacuum 2012, 86, 871-875, https://doi.org/10.1016/j.vacuum.2011.03.017.

11. Maleki-Ghaleh, H.; Shahzadeh, M.; Hoseinizadeh, S.A.; Arabi, A.; Aghaie, E.; Siadati, M.H. Evaluation of the photoelectro-catalytic behavior of nano-structured $\mathrm{ZnO}$ films fabricated by electrodeposition process. Mater. Lett. 2016, 169, 140-143, https://doi.org/10.1016/j.matlet.2016.01.090.

12. Zhu, L.; Li, Y.; Zeng, W. Hydrothermal synthesis of hierarchical flower-like ZnO nanostructure and its enhanced ethanol gas-sensing properties. Appl. Surf. Sci 2018, 427, 281-287, https://doi.org/10.1016/j.apsusc.2017.08.229.

13. Brahma, S.; Shivashankar, S.A. Microwave irradiation assisted rapid growth of ZnO nanorods over metal coated/electrically conducting substrate. Mater. Lett $\mathbf{2 0 2 0}, \quad 264, \quad 127370$, https://doi.org/10.1016/j.matlet.2020.127370.

14. Dwivedi, S.; Wahab, R.; Khan, F.; Mishra, Y.K.; Musarrat, J.; Al-Khedhairy, A.A. Reactive oxygen species mediated bacterial biofilm inhibition via zinc oxide nanoparticles and their statistical determination. PLOS ONE 2014, 9, e111289, https://doi.org/10.1371/journal.pone.0111289.

15. Sahu, K.; Kuriakose, S.; Singh, J.; Satpati, B.; Mohapatra, S. Facile synthesis of ZnO nanoplates and nanoparticle aggregates for highly efficient photocatalytic degradation of organic dyes. J. Phys. Chem. Sol. 2018, 121, 186-195, https://doi.org/10.1016/j.jpcs.2018.04.023.

16. Ghorbani, H.R.; Mehr, F.P.; Pazoki, H.; Rahmani, B.M. Synthesis of ZnO nanoparticles by precipitation method. Orient. J. Chem. 2015, 31, 1219-1221, https://doi.org/10. 13005/ojc/310281.

17. Gopal, V.R.V.; Kamila, S. Effect of temperature on the morphology of ZnO nanoparticles: a comparative study. Appl. Nanosci. 2017, 7, 75-82, https://doi.org/10.1007/s13204-017-0553-3.

18. Mahdavi, R.; Talesh, S.S.A. The effect of ultrasonic irradiation on the structure, morphology and photocatalytic performance of $\mathrm{ZnO}$ nanoparticles by sol-gel method. Ultrason. Sonochem. 2017, 39, 504510, https://doi.org/10.1016/j.ultsonch.2017.05.012.

19. Droepenu, E.K.; Wee, B.S.; Chin, S.F.; Kok, K.Ying.; Zaini, B.A.; Asare, E.A. Comparative evaluation of antibacterial efficacy of biological synthesis of $\mathrm{ZnO}$ nanoparticles using fresh leaf extract and fresh stembark of Carica papaya. Nano Biomed. Eng. 2019, 11, 264-271, https://doi.org/10.5101/nbe.v11i3.p264-271.

20. Ghanbari, M.; Bazarganipour, M.; Salavati-Niasari, M. Photodegradation and removal of organic dyes using cui nanostructures, green synthesis and characterization. Separa. Purific. Technol. 2017, 173, 27-36, https://doi.org/10.1016/j.seppur.2016.09.003.

21. Kalpana, V.N.; Rajeswari, V.D. A review on green synthesis, biomedical applications, and toxicity studies of ZnO NPs. Bioinorg. Chem. Appls 2018, 1-12, https://doi.org/10.1155/2018/3569758.

22. Chu, H.O.; Wang, Q. Shi, Y-J.; Song, S.; Liu, W.; Zhou, S.; Gibson, D.; Alajlani, Y.; Li. C. Structural, optical properties and optical modelling of hydrothermal chemical growth derived $\mathrm{ZnO}$ nanowires. Trans. Nonferrous Met. Soc. China 2020, 30, 191-199, https://doi.org/10.1016/S1003-6326(19)65191-5.

23. Rocha, L.S.R.; Foschini, C.R.; Silva, C.C.; Longo, E.; Simoes, A.Z. Novel ozone gas sensor based on ZnO nanostructures grown by the microwave-assisted hydrothermal route. Ceram. Int. 2016, 42, 4539-4545, https://doi.org/10.1016/j.ceramint.2015.11.145.

24. Li, X.; Wang, C.; Zhou, X.; Liu, J.; Sun, P.; Lu, G. Gas sensing properties of flower-like ZnO prepared by microwave-assisted technique. RSC Adv. 2014, 4, 47319-47324, https://doi.org/10.1039/C4RA07425D.

25. Vahidi, A.; Vaghari, H.; Najian, Y.; Najian, M.J.; Jafarizadeh-Malmiri, H. Evaluation of three different green fabrication methods for the synthesis of crystalline $\mathrm{ZnO}$ nanoparticles using Pelargonium zonale leaf extract. Green Proc. \& Synth. 2019, 8, 302-308, https://doi.org/10.1515/gps-2018-0097.

26. Satheshkumar, M.; Anand, B.; Muthuvel, A.; Rajarajan, M.; Mohana, V.; Sundaramanickam, A. Enhanced photocatalytic dye degradation and antibacterial activity of biosynthesized ZnO-NPs using curry leaves extract with coconut water. Nanotechnol. Environ. Eng. 2020, 5, 1-11, https://doi.org/10. 1007/s41204-02000093-x. 
27. Wojnarowicz, J.; Chudoba, T.; Lojkowski, W. A Review of microwave synthesis of zinc oxide nanomaterials: reactants, process parameters and morphologies. Nanomater. 2020, 10, 1086, 1-140, https://doi.org/10.3390/nano10061086.

28. Saberon, S.I.; Maguyon-Detras, M.C.; Migo, M.V.P.; Herrera, M.U.; Manalo, R.D. Microwave-assisted synthesis of zinc oxide nanoparticles on paper. Key Engineering Mater. 2018, 775, 163-168, https://doi.org/10.4028/www.scientific.net/KEM.775.163.

29. Yang, G.; Park, S.-J. Conventional and microwave hydrothermal synthesis and application of functional materials: A review. Mater. 2019, 12, 1177, https://doi.org/10.3390/ma12071177.

30. Jalouli, B.; Abbasi, A.; Khoei, M.S.M. A comment on: "Conventional and microwave hydrothermal synthesis and application of functional materials: A review”. Mater. 2019, 12, 3631, https://doi.org/10.3390/ma12213631.

31. Rana, K.; Rana, S. Microwave reactors: A brief review on its fundamental aspects and applications. Open Acc. Lib. J. 2014, 1, 1-20, https://doi.org/10.4236/OALIB.1100686

32. Kim, T.; Lee, J.; Lee, K.-H. Microwave heating of carbon-based solid materials. Carbon Lett. 2014, 15, 1524, https://doi.org/10.5714/CL.2014.15.1.015.

33. Mohan, S.; Vellakkat, M.; Aravind, A.; Rekha, U. Hydrothermal synthesis and characterization of zinc oxide nanoparticles of various shapes under different reaction conditions. Nano Express 2020, 1-25, https://doi.org/10.1088/2632-959X/abc813.

34. Ming, O.U.; Lin, M.A.; Limei, X.U.; Haizhen, L.I.; Zhuomei, Y.; Zhifeng, L.A.N. Microwave-assisted synthesis of hierarchical $\mathrm{ZnO}$ nanostructures and their photocatalytic properties. MATEC Web of Conferences 2016, 67, 1-7.

35. Suwanboon, S.; Klubnuan, S.; Jantha, N.; Amornpitoksuk, P.; Bangrak, P. Influence of alkaline solutions on morphology of $\mathrm{ZnO}$ prepared by hydrothermal method for using as photocatalyst and bactericidal agent. Mater. Lett. 2014, 115, 275-278, https://doi.org/10.1016/j.matlet.2013.10.066.

36. Zhu, L.; Li, Y.; Zeng, W. Hydrothermal synthesis of hierarchical flower-like ZnO nanostructure and its enhanced ethanol gas-sensing properties. Appl. Surf. Sci. 2018, 427, 281-287, https://doi.org/10.1016/j.apsusc.2017.08.229.

37. Zhou, Q.; Xie, B.; Jin, L.; Chen, W.; Li, J. Hydrothermal synthesis and responsive characteristics of hierarchical zinc oxide nanoflowers to sulfur dioxide. J. Nanotechnol. 2016, 1-6, https://doi.org/10.1155/2016/2083948.

38. Perillo, P.M.; Atia, M.N.; Rodríguez, D.F. Studies on the growth control of ZnO nanostructures synthesized by the chemical method. Revista Matéria 2018, 22, 1-7, https://doi.org/10.1590/S1517-707620180002.0467.

39. Alver, U.; Tanriverdi, A.; Akgul, O. Hydrothermal preparation of $\mathrm{ZnO}$ electrodes synthesized from different precursors for electrochemical supercapacitors. Synthetic Mater. 2016, 211, 30-34, https://doi.org/10.1016j.synthmet.2015.11.008.

40. Amin, G.; Asif, M.H.; Zainelabdin, A.; Zaman, S.; Nur, O.; Willander, M. Influence of pH, precursor concentration, growth time, and temperature on the morphology of $\mathrm{ZnO}$ nanostructures grown by the hydrothermal method. J. Nanomater. 2011, 1-10, https://doi.org/10.1155/2011/269692.

41. Sarangi, S. N., Acharya, S., and Biswal, S. K. (2018). Different morphology of ZnO nanostructures using hydrothermal and electro-deposition technique. Aspects in Mining and Mineral Science, 1, AMMS.000515, 92-93.

42. Li, J.; Wu, Q.; Wu, J. Synthesis of nanoparticles via solvothermal and hydrothermal methods. Handb. Nano. 2016, 295-328, https://doi.org/10.1007/978-3-319-15338-4_17.

43. Zak, A.K.; Razali, R.; Majid, W.H.A.; Darroudi, M. Synthesis and characterization of a narrow size distribution of zinc oxide nanoparticles. Int. J. Nanomed. 2011, 6, 1399-1403, https://doi.org/10.2147/IJN.S19693.

44. Bai, X.; Li, L.; Liu, H.; Tan, L.; Liu, T.; Meng, X. Solvothermal synthesis of ZnO nanoparticles and antiinfection application in vivo. ACS Appl. Mater. Interface 2015, 7, 1308-1317, https://doi.org/10.1021/am507532p.

45. Šarić, A.; Štefanić, G.; Dražić, G.; Gotić, M. Solvothermal synthesis of zinc oxide microspheres. J. Alloys Compd. 2015, 652, 91-99, https://doi.org/10.1016/j.jallcom.2015.08.200.

46. Šarić, A.; Gotić, M.; Štefanić, G.; Dražić, G. Synthesis of ZnO particles using water molecules generated in esterification reaction. J. Mol. Struct. 2017, 1140, 12-18, https://doi.org/10.1016/j.molstruc.2016.10.057.

47. Ludi, B.; Niederberger, M. Zinc oxide nanoparticles: Chemical mechanisms and classical and non-classical crystallization. Dalton Trans. 2013, 42, 12554-12568, https://doi.org/10.1039/C3DT50610J. 
48. Zare, M.; Namratha, K.; Byrappa, K.; Surendra, D.M.; Yallappa, S.; Hungund, B. Surfactant assisted solvothermal synthesis of $\mathrm{ZnO}$ nanoparticles and study of their antimicrobial and antioxidant properties. $J$. Mater. Sci. Technol. 2018, 1059, 1-9, https://doi.org/10.1016/j.jmst.2017.09.014.

49. Wojnarowicz, J.; Chudoba, T.; Gierlotka, S.; Sobczak, K.; Lojkowski, W. Size control of cobalt-doped ZnO nanoparticles obtained in microwave solvothermal synthesis. Crystals 2018, 8, 179, 1-18, https://doi.org/10.3390/cryst8040179.

50. Wojnarowicz, J.; Chudoba, T.; Koltsov, I.; Gierlotka, S.; Dworakowska, S. Lojkowski, W. Size control mechanism of $\mathrm{ZnO}$ nanoparticles obtained in microwave solvothermal synthesis. Nanotechnol. 2018, 29, 065601, https://doi.org/10.1088/1361-6528/aaa0ef.

51. Wojnarowicz, J.; Opalinska, A.; Chudoba, T.; Gierlotka, S.; Mukhovskyi, R.; Pietrzykowska, E.; Sobczak, K.; Lojkowski, W. Effect of water content in ethylene glycol solvent on the size of ZnO nanoparticles prepared using microwave solvothermal synthesis. J. Nanomater. 2016, 1-15, https://doi.org/10.1155/2016/2789871.

52. Wu, M.-L.; Chen, D.-H.; Huang, T.C. Preparation of Pd/Pt bimetallic nanoparticles in water/AOT/isooctane microemulsion. J. Colloid and Interface Sci. 2001, 243, 102-108, https://doi.org/10.1006/jcis.2001.7887.

53. Brahma, S.; Jaiswal, P.; Suresh, K.S.; Lo, K.-Y.; Suwas, S.; Shivashankar, S.A. Effect of substrates and surfactants over the evolution of crystallographic texture of nanostructured $\mathrm{ZnO}$ thin films deposited through microwave irradiation. Thin Solid Films 2015, 593, 81-90, https://doi.org/10.1016/j.tsf.2015.09.005.

54. Xin, Z.; Li, L.; Zhang, X.; Zhang, W. Microwave-assisted hydrothermal synthesis of chrysanthemum-like $\mathrm{Ag} / \mathrm{ZnO}$ prismatic nanorods and their photocatalytic properties with multiple modes for dye degradation and hydrogen production. RSC Adv. 2018, 8, 6027-6038, https://doi.org/10.1039/C7RA12097D.

55. Shen, X.; Sun, J.; Zhu, G.; Ji, Z.; Chen, Z.; Li, N. Morphological syntheses of ZnO nanostructures under microwave irradiation. J. Mater. Sci. 2013, 48, 2358-2364, https://doi.org/10.1007/s10853-012-7017-7.

56. Razali, R.; Zak, A.K.; Majid, W.H.A.; Darroudi, M. Solvothermal synthesis of microspheres ZnO nanostructures in DEA media. Ceram. Int. 2011, 37, 3657-3663, https://doi.org/10.1016/j.ceramint.2011.06.026.

57. Idiawati, R.; Mufti, N.; Taufiq, A.; Wisodo, H.; Laila, I.K.R.; Fuad, A. Effect of growth time on the characteristics of $\mathrm{ZnO}$ nanorods. IOP Conf. Series: Mater. Sci. Eng. 2017, 202, 012050, https://doi.org/10.1088/1757-899X/202/1/012050.

58. de Peres, M.L.; Delucis, R.A.; Amico, S.C.; Gatto, D.A. Zinc oxide nanoparticles from microwave-assisted solvothermal process: Photocatalytic performance and use for wood protection against xylophagous fungus. Nanomater \& Nanotechnol. 2019, 9, 1-8, https://doi.org/10.1177/1847980419876201.

59. Dimitriev, Y.; Ivanova, Y.; Iordanova, R. History of sol-gel science and technology (review). J. Univ. Chem. Technol. \& Metallu. 2008, 43, 181-192.

60. Parihar, V.; Raja, M.; Paulose, R. A brief review of structural, electrical and electrochemical properties of zinc oxide nanoparticles. Rev. Adv. Mater. Sci. 2018, 53, 119-130, https://doi.org/10.1515/rams-2018-0009.

61. Catauro, M.; Tranquillo, E.; Poggetto, G.D.; Pasquali, M.; Dell'Era, A.; Ciprioti, S. Influence of the heat treatment on the particles size and on the crystalline phase of $\mathrm{TiO}_{2}$ synthesized by sol-gel method. Materials 2018, 11, 2364, https://doi.org/10.3390/ma11122364.

62. Livage, J.; Henry, M.; Sanchez, C. Sol-gel chemistry of transition metal oxides. Prog. Solid State Chem. 1988. 18, 259-341, https://doi.org/10.1016/0079-6786(88)90005-2.

63. Parashar, M.; Shukla, V.K.; Singh, R. Metal oxides nanoparticles via sol-gel method: a review on synthesis, characterization and applications. J. Mater. Sci: Mater Electron, 2020, 31, 3729-3749, https://doi.org/10.1007/s10854-020-02994-8.

64. Singh, G.; Singh, S.P. Synthesis of zinc oxide by sol-gel method and to study its structural properties. AIP Conference Proceedings 2020, 2220, 020184, https://doi.org/10.1063/5.0001593.

65. Chen, X.; Wu, Z.; Liu, D.; Gao, Z. Preparation of ZnO photocatalyst for the efficient and rapid photocatalytic degradation of azo dyes. Nanoscale Res. Lett. 2017, 12, 1-10, https://doi.org/10.1186/s11671-0711904-4.

66. Shaikh, R.S.; Ravangave, L.S. Effect of reaction time on some characterization of $\mathrm{ZnO}$ nanoparticles. Int. Res. J. Sci. \& Eng., Special Issue A2 2018, 187-191, ISSN: 2322-0015.

67. Hasnidawani, J.N.; Azlina, H.N.; Norita, H.; Bonnia, N.N.; Ratim, S.; Ali, E.S. Synthesis of ZnO nanostructures using sol-gel method. Procedia Chem. 2016, 19, $211 \quad$ - 216, https://doi.org/10.1016/j.proche.2016.03.095. 
68. Iwamura, T.; Goto, S.; Sakaguchi, M.; Chujo, Y. Synthesis of submicrometer zinc oxide particles and zinc oxide nanowires using microwave irradiation. Chem. Lett. 2016, 45, 508-510, https://doi.org/10.1246/cl.160081.

69. Iwamura, T.; Adachi, K.; Chujo, Y. Simple and rapid eco-friendly synthesis of cubic octamethylsilsesquioxane using microwave irradiation. Chem. Lett. 2010, 39, 354-355, https://doi.org/10.1246/cl.2010.354.

70. Vanaja, A.; Rao, K.S. Effect of solvents on particle structure, morphology and optical properties of zinc oxide nanoparticles. Int. J. Adv. Mater. Sci. Eng. (IJAMSE) 2015, 4, 1-8, https://doi.org/10.14810/ijamse.2015.4201.

71. Borm, P.J.A.; Robbins, D.; Haubold, S.; Kuhlbusch, T.; Fissan, H.; Donaldson, K.; Schins, R.; Stone, V.; Kreyling, W.; Lademann, J.; Krutmann, J.; Warheit, D.; Oberdorster, E. The potential risk of nanomaterials: A review carried out for ECETOC. Part. Fibre Toxicol. 2006, 3, 11-45, https://doi.org/10.1186/1743-89773-11.

72. Khan, M.F.; Ansari, A.H.; Hameedullah, M.; Ahmad, E.; Husain, F.M.; Zia, Q.; Baig, U.; Zaheer, M.R.; Alam, M.M.; Khan, A.M.; AlOthman, Z.A.; Ahmad, I.; Ashraf, G.M.; Aliev, G. Sol-gel synthesis of thornlike $\mathrm{ZnO}$ nanoparticles endorsing mechanical stirring effect and their antimicrobial activities: Potential role as nano-antibiotics. Scientific Reports 2016, 6, 1-12, https://doi.org/10.1038/srep27689.

73. Brintha, S.R.; Ajitha, M. Synthesis and characterization of $\mathrm{ZnO}$ nanoparticles via aqueous solution, sol-gel and hydrothermal methods. J. Appl. Chem. (IOSR-JAC) 2015, 8, 66-72.

74. Sayari, A.; EI Mir, L. Structural and characterization of Ni and Al Co-doped ZnO nanopowders synthesized via the sol-gel process. Kona Powder and Particle Journal 2016, 32, 154-162, https://doi.org/10.14356/kona.2015003

75. Shohany, B.G.; Zak, A.K. Doped ZnO nanostructures with selected elements - Structural, morphology and optical properties: A review. Ceramics Int. 2019, 46, 5507-5520, https://doi.org/10.1016/j.ceramint.2019.11.051.

76. Yousefi, R.; Zak, A.K.; Jamali-Sheini, F. The effect of group-I elements on the structural and optical properties of $\mathrm{ZnO}$ nanoparticles, Ceramics Int. 2013, 39, 1371-1377, https://doi.org/10.1016/j.ceramint.2012.07.076.

77. Hameed, A.S.H.; Karthikeyan, C.; Sasikumar, S.; Kumar, V.S.; Kumaresan, S.; Ravi, G. Impact of alkaline metal ions $\mathrm{Mg}^{2+}, \mathrm{Ca}^{2+}, \mathrm{Sr}^{2+}$ and $\mathrm{Ba}^{2+}$ on the structural, optical, thermal and antibacterial properties of $\mathrm{ZnO}$ nanoparticles prepared by the co-precipitation method. J. Mater. Chem. B 2013, 1, 5950-5962, https://doi.org/10.1039/C3TB21068E.

78. Zak, A.K.; Majid, W.H.A.; Abrishami, M.E.; Yousefi, R.; Parvizi, R. Synthesis, magnetic properties and Xray analysis of $\mathrm{Zn}_{0.97} \mathrm{X}_{0.03} \mathrm{O}$ nanoparticles $(\mathrm{X}=\mathrm{Mn}, \mathrm{Ni}$, and $\mathrm{Co}$ ) using Scherrer and size-strain plot methods. Solid State Sci. 2012, 14, 488-494, https://doi.org/10.1016/J.solidstatesciences.2012.01.019.

79. Kaur, P.; Kumar, S.; Chen, C.L.; Hsu, Y-Y.; Chan, T-S.; Dong, C-L.; Srivastava, C.; Singh, A.; Rao, S.M. Investigations on structural, magnetic and electronic structure of Gd-doped $\mathrm{ZnO}$ nanostructures synthesized using sol-gel technique. Appl. Phys. A 2016, 122, 161, https://doi.org/10.1007/s00339-016-9707-5.

80. Barreto, G.; Morales, G.; Cañizo, A.; Eyler, N. Microwave assisted synthesis of ZnO tridimensional nanostructures. Procedia Mater. Sci. 2015, 8, 535 - 540, https://doi.org/10.1016/j.mspro.2015.04.106.

81. Hasanpoor, M.; Aliofkhazraei, M.; Delavari, H. Microwave-assisted synthesis of zinc oxide nanoparticles. Procedia Mater. Sci. 2015, 11, 320-325, https://doi.org/10.1016/j.mspro.2015.11.101.

82. Byzynski, G.; Pereira, A.P. Volanti, D.P.; Ribeiro, C.; Longo, E. High-performance ultraviolet-visible driven $\mathrm{ZnO}$ morphologies photocatalyst obtained by microwave-assisted hydrothermal method. J. Photochem. \& Photobio. A: Chem. 2018, 353, 358-367, https://doi.org/10.1016/j.jphotochem.2017.11.032.

83. Sadhukhan, P.; Kundu, M.; Rana, S.; Kumar, R.; Das, J.; Sil, P.C. Microwave induced synthesis of ZnO nanorods and their efficacy as a drug carrier with profound anticancer and antibacterial properties. Toxicol Reports 2019, 6, 176-185, https://doi.org/10.1016/j.toxrep.2019.01.006.

84. Markovic, S.; Simatovic, I.S.; Ahmetovic, S.; Veselinovic, L.; Stojadinovic, S.; Rac, V.; Skapin, S.D.; Bogdanovic, D.B.; Castvan, I.J.; Uskokovic, D. Surfactant-assisted microwave processing of ZnO particles: a simple way for designing the surface-to-bulk defect ratio and improving photo(electro)catalytic properties. RSC Adv. 2019, 9, 17165-17178, https://doi.org/10.1039/C9RA02553G.

85. Thi, V.H.T.; Lee, B-K. Great improvement on tetracycline removal using $\mathrm{ZnO}$ rod-activated carbon fiber composite prepared with a facile microwave method. J. Hazard. Mater. 2016, 324, 329-339, https://doi.org/10.1016/j.jhazmat.2016.10.066. 
86. Sun, H.; Sun, L.; Sugiura, T.; White, M.S.; Stadler, P.; Sariciftci, N.S.; Masuhara, A.; Yoshida, T. Microwaveassisted hydrothermal synthesis of structure-controlled $\mathrm{ZnO}$ nanocrystals and their properties in dyesensitized solar cells. Electrochem. 2017, 85, 253-261, https://doi.org/10.5796/electrochemistry.85.253.

87. Wannapop, S.; Somdee, A.; Thongtem, T.; Thongtem, S. Synthesis of ZnO nanostructures by microwave irradiation for energy conversion material in for dye sensitized solar cells and materials for photocatalytic dye degradation applications. J. Ceram. Soc. Japan 2019, 127, 428-434, https://doi.org/10.2109/jcersj2.19015.

88. Pulit-Prociak, J.; Banach, M. Effect of process parameters on the size and shape of nano- and micrometric zinc oxide. Acta Chim. Slov. 2016, 63, 317-322, https://doi.org/10.17344/acsi.2016.2245.

89. Pauzi, N.; Zain, N.M.; Yusof, N.A.A. Microwave-assisted synthesis of ZnO nanoparticles stabilized with Gum Arabic: effect of microwave irradiation time on $\mathrm{ZnO}$ nanoparticles size and morphology. Bullet. Chem. React. Eng. \& Catal. 2019, 14, 182-188, https://doi.org/10.9767/bcrec.14.1.3320.182-188.

90. Gray, R.J.; Jaafar, A.H.; Verrelli, E.; Kemp, N.T. Method to reduce the formation of crystallites in ZnO nanorod thin-films grown via ultra-fast microwave heating. Thin Solid Films 2018, 662, 116-122, https://doi.org/10.1016/j.tsf.2018.07.03.034.

91. Marzouqi, F.A.; Adawi, H.A.; Qi, K.; Liu, S-y.; Kim, Y.; Selvaraj, R. A green approach to the microwaveassisted synthesis of flower-like $\mathrm{ZnO}$ nanostructures for reduction of $\mathrm{Cr}(\mathrm{VI})$. Toxicol. Environ. Chem. 2019, 101, 1-12, https://doi.org/10.1080/02772248.2019.1635602.

92. Wasly, H.S.; El-Sadek, M.S.A.; Henini, M. Influence of reaction time and synthesis temperature on physical properties of $\mathrm{ZnO}$ nanoparticles synthesized by hydrothermal method. Appl. Phys. A 2018, 124, 76, https://doi.org/10.1007/s00339-017-1482-4.

93. Bayrami, A.; Ghorbani, E.; Pouran, S.R.; Habibi-Yangjeh, A.; Khataee, A.; Bayrami, M. Enriched zinc oxide nanoparticles by Nasturtium officinale leaf extract: Joint ultrasound-microwave-facilitated synthesis, characterization, and implementation for diabetes control and bacterial inhibition. Ultrasonics-Sonochemistry 2019, 58, 104613, 1-8, https://doi.org/10.1016/j.ultsonch.2019.104613.

94. Bayrami, A.; Parvinroo, S.; Habibi-Yangjeh, A.; Pouran, S.R. Bio-extract-mediated ZnO nanoparticles: microwave-assisted synthesis, characterization and antidiabetic activity evaluation. Artificial Cells, Nanomed. \& Biotechnol. 2018, 46, 730-739, https://doi.org/10.1080/21691401.2017.1337025.

95. Chae, H.U.; Rana, A.H.S.; Park, Y-J.; Kim, H-S. High-speed growth of ZnO nanorods in preheating condition using microwave-assisted growth method. J. Nanosci. Nanotechnol. 2018, 18, 2041-2044, https://doi.org/10.1166/jnn.2018.14971.

96. Chauhan, D.S.; Gopal, C.S.A.; Kumar, D.; Mahato, N.; Quraishi, M.A.; Cho, M.H. Microwave induced facile synthesis and characterization of $\mathrm{ZnO}$ nanoparticles as efficient antibacterial agents. Mater. Discovery 2018, 11, 19-25, https://doi.org/10.1016/j.md.2018.05.001.

97. Fang, M.; Liu, Z.W. Controllable size and photoluminescence of $\mathrm{ZnO}$ nanorod arrays on Si substrate prepared by microwave-assisted hydrothermal method, Ceramics Int. 2017, 43, 6955-6962, https://doi.org/10.1016/j.ceramint.2017.02.119.

98. Giridhar, M.; Naik, H.S.B.; Sudhamani, C.N.; Prabakara, M.C.; Kenchappa, R.; Venugopal, N.; Patil, S. Microwave-assisted synthesis of water-soluble styrylpyridine dye-capped zinc oxide nanoparticles for antibacterial applications. J. Chin. Chem, Soc. 2019, 1-8, https://doi.org/10.1002/jccs.201900029.

99. Goswami, S.R.; Singh, M. Microwave-mediated synthesis of zinc oxide nanoparticles: a therapeutic approach against Malassezia species. The Institution of Engineering and Technology Biotechnology 2018, 1-6, https://doi.org/10.1049/iet-nbt.2018.0007.

100.Hirai, Y.; Furukawa, K.; Sun, H.; Matsushima, Y.; Shito, K.; Masuhara, A.; Ono, R.; Shimbori, Y.; Shiroishi, H.; White, M.S.; Yoshida, T. Microwave-assisted hydrothermal synthesis of ZnO and Zn-terephthalate hybrid nanoparticles employing benzene dicarboxylic acids. Microsyst Technol. 2017, 1-10, https://doi.org/10.1007/s00542-017-3392-y.

101.Sooksaen, P.; Chuankrerkkul, N. Morphology-design and semiconducting characteristics of zinc oxide nanostructures under microwave irradiation. Integrated Ferroelectrics 2017, 91-102, https://doi.org/10.1080/10584587.2017.1285194.

102.Jaafar, N.F.; Najman, A.M.M.; Marfura, A.; Jusoh, N.W.C. Strategies for the formation of oxygen vacancies in zinc oxide nanoparticles used for photocatalytic degradation of phenol under visible light irradiation. $J$. Photochem. \& Photobio A: Chem. 2019, 388, 112202, 1-10, https://doi.org/10.1016/j.jphotochem.2019.112202. 
103.Malik, L.A.; Bashir, A.; Manzoor, T.; Pandith, A.H. Microwave-assisted synthesis of glutathione-coated hollow zinc oxide for the removal of heavy metal ions from aqueous systems. RSC Adv. 2019, 9, $15976-$ 15985, https://doi.org/10.1039/C9RA00243.

104.Al-Sabahi, J.; Bora, T.; Al-Abri, M.; Dutta, J. Controlled defects of zinc oxide nanorods for efficient visible light photocatalytic degradation of phenol. Mater. 2016, 9, 238, 1-10, https://doi.org/10.3390/ma9040238.

105.Pimentel, A.; Ferreira, S.H.; Nunes, D.; Calmeiro, T.; Martins, R.; Fortunato, E. Microwave synthesized ZnO nanorod arrays for UV sensors: A seed layer annealing temperature study. Mater. 2016, 9, 299, 1-15, https://doi.org/10.3390/ma9040299.

106.Tan, S.T.; Tan, C.H.; Chong, W.Y.; Yap, C.C.; Umar, A.A.; Ginting, R.T.; Lee, H.B.; Lim, K.S.; Yahaya, M.; Salleh, M.M. Microwave-assisted hydrolysis preparation of highly crystalline ZnO nanorod array for room temperature photoluminescence-base CO gas sensor. Sensors and Actuators B 2016, 227, 304-312, https://doi.org/10.1016/j.snb.2015.12.058

107.Song, H.; Zhu, K.; Liu, Y.; Zhai, X. Microwave-assisted synthesis of ZnO and its photocatalytic activity in degradation of CTAB. Rus. J. Phys. Chem. A 2017, 91, 59-62, https://doi.org/10.1134/S003602441701.

108. Nandi, A.; Nag, P.; Saha, H.; Majumdar, S. Precursor dependent morphologies of microwave assisted ZnO nanostructures and their VOC detection properties. Mater. Today: Proceedings 2018, 5, 9831-9838, https://doi.org/10.1016/j.matpr.2017.10.174.

109.Papadaki, D.; Foteinis, S.; Mhlongo, G.H.; Nkosi, S.S.; Motaung, D.E.; Ray, S.S.; Tsoutsos, T.; Kiriakidis, G. Life cycle assessment of facile microwave-assisted zinc oxide ( $\mathrm{ZnO})$ nanostructures. Sci. Tot. Environ. 2017, 586, 566-575, https://doi.org/10.1016/j.scitotenv.2017.02.019.

110.Quirino, M.R.; Oliveira, M.J.C.; Keyson, D.; Lucena, G.L.; Oliveira, J.B.L.; Gama, L. Synthesis of zinc oxide by microwave hydrothermal method for application to transesterification of soyabean oil (biodiesel). Mater. Chem. Phys. 2016, 185, 24-30, https://doi.org/10.1016/j.matchemphys.2016.09.06.

111.Witkowski, B.S.; Dluzewski, P.; Kaszewski, J.; Sylwia, L.W. Ultra-fast epitaxial growth of ZnO nano/microrods on a GaN substrate, using the microwave-assisted hydrothermal method. Mater. Chem. Phys. 2018, 205, 16-22, https://doi.org/10.1016/j.matchemphys.2017.11.00.

112. Thankachan, R.M.; Joy, N.; Abraham, J.; Kalarikkal, N.; Thomas, S.; Oluwafemi, O.S. Enhanced photocatalytic performance of $\mathrm{ZnO}$ nanostructures produced via a quick microwave assisted route for the degradation of rhodamine in aqueous solution. Mater. Res. Bull. 2017, 85, 131-139, https://doi.org/10.1016/j.materresbull.2016.09.009.

113.Husham, M.; Hamidon, M.N.; Paiman, S.; Abuelsamen, A.A.; Farhat, O.F.; Al-Dulaimi, A.A. Synthesis of $\mathrm{ZnO}$ nanorods by microwave-assisted chemical-bath deposition for highly sensitive self-powered UV detection application. Sensors and Actuators A 2017, 263, 166-173, https://doi.org/10.1016/j.sna.2017.05.041.

114.Salah, N.; AL-Shawafi, W.M.; Alshahrie, A.; Baghdadi, N.; Soliman, Y.M.; Memic, A. Size controlled, antimicrobial $\mathrm{ZnO}$ nanostructures produced by the microwave assisted route. Mater. Sci. \& Eng. C 2019, 99, 1164-1173, https://doi.org/10.1016/j.msec.2019.02.077.

115.Sakata, K.; Macounová, K.M., Nebel, R.; Krtil, P. pH dependent ZnO nanostructures synthesized by hydrothermal approach and surface sensitivity of their photoelectrochemical behaviour. SN Appl. Sci. 2020, 2, 203, 1-8, https://doi.org/10.1007/s42452-020-1975-1.

116. Sekhar, M.C.; Ramana, M.V. Instant synthesis of $\mathrm{ZnO}$ nanoparticles by microwave hydrothermal method. Int. J. NanoSci. Nanotechnol. 2017, 8, 17-23, ISSN: 0974-3081.

117.Šarić, A.; Despotović, I.; Štefanić, G. Alcoholic solvent influence on ZnO synthesis: A joint experimental and theoretical study. J. Phys. Chem. C 2019, 123, 29394-2940, https://doi.org/10.1021/acs.jpcc.9b07411.

118. Ahammed, K.R.; Ashaduzzaman, M.; Paul, S.C.; Nath, M.R.; Bhowmik, S.; Saha, O.; Rahaman, M.M.; Bhowmik, S.; Aka, T.D. Microwave assisted synthesis of zinc oxide ( $\mathrm{ZnO})$ nanoparticles in a noble approach: utilization for antibacterial and photocatalytic activity. SN Appl. Sci. 2020, 2, 955, 1-14, https://doi.org/10.1007/s42452-020-2762-8.

119.Xiangyang, B.; Linlin, L.; Huiyu, L.; Longfei, T.; Tianlong, L.; Xianwei, M. Small molecule ligand solvothermal synthesis of $\mathrm{ZnO}$ nanoparticles and anti-infection application in vivo. ACS Appl. Mater. Interfaces 2015, 7, 1308-1317, https://doi.org/10.1021/am507532p.

120.Angaiah, S.; Arunachalam, S.; Murugadoss, V.; Vijayakumar, G. A Facile Polyvinylpyrrolidone assisted solvothermal synthesis of zinc oxide nanowires and nanoparticles and their influence on the photovoltaic performance of dye sensitized solar cell. ES Energy Environ. 2019, 4, 59-65, https://doi.org/10.30919/esee8c280. 
121.Feng, W.; Huang, P.; Wang, B.; Wang, C.; Wang, W.; Wang, T.; Chen, S.; Lv, R.; Qin, Y.; Ma, J. Solvothermal synthesis of $\mathrm{ZnO}$ with different morphologies in dimethylacetanamide media. Ceramics Int. 2015, 42, 2250-2256, https://doi.org/10.1016/j.ceramint.2015.10.018.

122.Mao, Y.; Li, Y.; Zou, Y.; Shen, X.; Zhu, L.; Liao, G. Solvothermal synthesis and photocatalytic properties of $\mathrm{ZnO}$ micro/nanostructures. $\quad$ Ceramics $\quad$ Int. $\quad \mathbf{2 0 1 8 ,}$ 45, 1724-1729, https://doi.org/10.1016/j.ceramint.2018.10.054.

123. Wojnarowicz, J.; Chudoba, T.; Gierlotka, S.; Lojkowski, W. Effect of microwave radiation power on the size of aggregates of ZnO NPs prepared using microwave solvothermal synthesis. Nanomater. 2018, 8, 343, 117, https://doi.org/10.3390/nano8050343.

124. Nuraqeelah, M.S.; Wee, B.S.; Chin, S.F.; Kok, K.Y. Synthesis and characterization of zinc oxide nanoparticles with small particle size distribution. Acta Chim. Slov. 2018, 65, 578-585, https://doi.org/10.17344/acsi.2018.4213.

125. Yao, Q.; Wang, C.; Fan, B.; Wang, H.; Sun, Q.; Jin, C.; Zhang, H. One-step solvothermal deposition of ZnO nanorod arrays on a wood surface for robust superamphiphobic performance and superior ultraviolet resistance. Scientific Reports 2016, 6, 35505, 1-11, https://doi.org/10.1038/srep35505.

126. Bhatta, L.K.G.; Bhatta, U.M.; Venkatesh, K. Facile microwave-assisted synthesis of zinc oxide and characterization. J. Scienti. Industr. Res. 2019, 78, 173-176, ISSN: 0975-1084.

127.Jamatia, T.; Skoda, D.; Urbanek, P.; Munster, L.; Sevcik, J.; Kuritka, I. Microwave-assisted particle sizecontrolled synthesis of $\mathrm{ZnO}$ nanoparticles and its application in fabrication of PLED device. Applied Nanotechnology and Nanoscience International Conference (ANNIC 2018), IOP Conf. Series: J. Phys: Conf. Series, 1310 2019, 012012, https://doi.org/10.1088/1742-6596/1310/1/012012.

128. Karthikeyan, L.; Akshaya, M.V.; Basu, P.K. Microwave assisted synthesis of ZnO and Pd-ZnO nanospheres for UV photodetector. Sensors and Actuators A: Physical 2017, 264, 90-95, https://doi.org/10.1016/j.sna.2017.06.013.

129.Kumar, V.; Gohain, M.; Som, S.; Kumar, V.; Bezuindenhoudt, B.C.B.; Swart, H.C. Microwave assisted synthesis of $\mathrm{ZnO}$ nanoparticles for lighting and dye removal application. Physica B: Condensed Matter 2016, 480, 36-41, https://doi.org/10.1016/j.physb.2015.07.020.

130.Pimentel, A.; Rodrigues, J.; Duarte, P.; Nunes, D.; Costa, F.M.; Monteiro, T.; Martins, R.; Fortunato, E. Effect of solvents on $\mathrm{ZnO}$ nanostructures synthesized by solvothermal method assisted by microwave radiation: a photocatalytic study. J. Mater. Sci. 2015, 50, 5777-5787, https://doi.org/10.1007/s10853-0159125-7.

131.Saloga, P.E.J.; Thünemann, A.F. Microwave-assisted synthesis of ultrasmall zinc oxide nanoparticles. Langmuir 2019, 35, 12469-12482, https://doi.org/10.1021/acs.langmuir.9b01921.

132.Skoda, D.; Urbanek, P.; Sevcik, J.; Munster, L.; Antos, J.; Kuritka, I. Microwave-assisted synthesis of colloidal $\mathrm{ZnO}$ nanocrystals and their utilization in improving polymer light emitting diodes efficiency. Mater. Sci. Eng. B 2018, 232, 22-32, https://doi.org/10.1016/j.mseb.2018.10.013.

133.Ikono, R.; Akwalia, P.R.; Siswanto, Wahyu, B.W.; Sukarto, A.; Rochman, N.T. Effect of pH variation on particle size and purity of nano zinc oxide synthesized by sol-gel method. Int. J. Eng. \& Technol. (IJETIJENS) 2012, 12, 5-9.

134.Divya, B.; Karthikeyan, C.; Rajasimman, M. Chemical synthesis of zinc oxide nanoparticles and its application of dye decolourization. Int. J. Nanosci. Nanotechnol. 2018, 14, 267-275.

135.Prasad, T.; Halder, S.; Goyat, M.S.; Dhar, S.S. Morphological dissimilarities of ZnO nanoparticles and its effect on thermo-physical behavior of epoxy composites. Polymer Composites 2018, 39, 135-145, https://doi.org/10.1002/pc.23914.

136. Ashraf, R., Riaz, S., Khaleeq-ur-Rehman, M., and Naseem, S. (2013). Synthesis and characterization of ZnO nanoparticles. The 2013 World Congress on Advances in Nano, Biomechanics, Robotics and Energy Research (ANBRE13), Seoul, Korea, August, 25-28, 2013.

137. Muñoz-Fernandez, L.; Sierra-Fernandez, A.; Miloševic, O.; Rabanal, M.E. Solvothermal synthesis of $\mathrm{Ag} / \mathrm{ZnO}$ and $\mathrm{Pt} / \mathrm{ZnO}$ nanocomposites and comparison of their photocatalytic behaviors on dyes degradation. Adv. Powd. Technol. 2016, 27, 983-993, https://doi.org/10.1016/j.apt.2016.03.021.

138. Vakili, B.; Shahmoradi, B.; Maleki, A.; Safari, M.; Yang, J.; Pawar, R.R.; Lee, S-M. Synthesis of immobilized cerium doped $\mathrm{ZnO}$ nanoparticles through the mild hydrothermal approach and their application in the photodegradation of synthetic wastewater. J. Mol. Liq. 2018, 280, 230-237, https://doi.org/10.1016/j.molliq.2018.12.103. 
139.Gupta, R.; Eswar, N.K.; Modak, J.M.; Madras, G. Effect of morphology of zinc oxide in ZnO-CdS-Ag tenary nanocomposite towards photocatalytic inactivation of E. coli under UV and visible light. Chem. Eng. J. 2017, 307, 966-980, https://doi.org/10.1016/j.cej.2016.08.142.

140. Yuvaraj, S.; Fernandez, A.C.; Sundararajan, M.; Dash, C.S.; Sakthivel, P. Hydrothermal synthesis of ZnOCdS nanocomposites: Structural, optical and electrical behaviour. Ceramics Int. 2020, 46, 391-402, https://doi.org/10.1016/j.ceramint.2019.08.274.

141. Manavalan, S.; Veerakumar, P.; Chen, S-M.; Lin, K-C. Three-dimensional zinc oxide nanostars anchored on graphene oxide for voltammetric determination of methyl parathion. Microchimica Acta 2020, 187, 1-13, https://doi.org/10.1007/s00604-019-4031-3.

142.Rakkesh, R.A.; Durgalakshmi, D.; Karthe, P.; Balakumar, S. Anisotropic growth and strain-induced tunable optical properties of $\mathrm{Ag}-\mathrm{ZnO}$ hierarchical nanostructures by a microwave synthesis method. Mater. Chem. Phys. 2020, 244, 122720, https://doi.org/10.1016/j.matchemphys.2020.122720.

143.Xin, Z.; Li, L.; Zhang, X.; Zhang, W. Microwave-assisted hydrothermal synthesis of chrysanthemum-like $\mathrm{Ag} / \mathrm{ZnO}$ prismatic nanorods and their photocatalytic properties with multiple modes for dye degradation and hydrogen production. RSC Adv. 2018, 8, 6027, https://doi.org/10.1039/C7RA12097D.

144.Sankar ganesh, R.; Navaneethan, M.; Mani, G.K.; Ponnusamy, S.; Tsuchiya, K.; Muthamizhchelvan, C.; Kawasaki, S.; Hayahawa, Y. Influence of Al doping on structural, morphological, optical and gas sensing properties of $\mathrm{ZnO}$ nanorods. J. Alloys \& Compounds 2017, 698, 555-564, https://doi.org/10.1016/j.jallcom.2016.12.187.

145.Gabriela, B.; Camila, M.; Volanti, D.P.; Ferrer, M.M.; Gouveia, A.F.; Cauê, R.; Juan, A.; Elson, L. The interplay between morphology and photocatalytic activity in $\mathrm{ZnO}$ and $\mathrm{N}$-doped $\mathrm{ZnO}$ crystals. Mater. \& Design 2017, 120, 363-375, https://doi.org/10.1016/j.matdes.2017.02.020.

146.Wojnarowicz, J.; Omelchenko, M.; Szczytko, J.; Chudoba, T.; Gierlotka, S.; Majhofer, A.; Twardowski, A.; Lojkowski, W. Structural and magnetic properties of Co-Mn codoped $\mathrm{ZnO}$ nanoparticles obtained by microwave solvothermal synthesis. Crystals 2018, 8, 410, https://doi.org/10.3390/cryst8110410.

147.Gao, X.; Zhang, T.; Wu, Y.; Yang, G.; Tan, M.; Li, X.; Xie, H.; Pan, J.; Tan, Y. Isobutanol synthesis from syngas on $\mathrm{Zn}-\mathrm{Cr}$ based catalysts: New insights into the effect of morphology and facet of $\mathrm{ZnO}$ nanocrystal. Fuel 2018, 217, 21-30, https://doi.org/10.1016/j.fuel.2017.12.065.

148. Aksoy, S.; Caglar, Y. Synthesis of Mn doped ZnO nanopowders by MW-HTS and its structural, morphological and optical characteristics. J. Alloys and Compounds 2019, 781, 929-935, https://doi.org/10.1016/j.jallcom.2018.12.101.

149.Wojnarowicz, J.; Mukhovskyi, R.; Pietrzykowska, E.; Kusnieruk, S.; Mizeracki, J.; Lojkowski, W. Microwave solvothermal synthesis and characterization of manganese-doped $\mathrm{ZnO}$ nanoparticles. Beilstein J. Nanotechnol. 2016, 7, 721-732, https://doi.org/10.3762/bjnano.7.64.

150.Guruvammal, D.; Selvaraj, S.; Sundar, S.M. Structural, optical and magnetic properties of Co doped ZnO DMS nanoparticles by microwave irradiation method. J. Magnetism and Magnetic Mater. 2018, 452, 335342, https://doi.org/10.1016/j.jmmm.2017.12.097.

151.Jayathilake, D.S.Y.; Peiris, T.A.N.; Sagu, J.S.; Potter, D.B.; Wijayantha, K.G.U.; Carmalt, C.J.; Southee, D.J. Microwave-assisted synthesis and processing of Al-doped, Ga-doped, and Al, Ga-codoped $\mathrm{ZnO}$ for the pursuit of optimal conductivity for transparent conducting film fabrication. ACS Sustainable Chem. Eng. 2017, 5, 4820-4829, https://doi.org/10.1021/acssuschemeng.7b00263.

152.Kadam, A.N.; Kim, T.G.; Shin, D-S.; Garadkar, K.M.; Park, J. Morphological evolution of Cu doped ZnO for enhancement of photocatalytic activity. J. Alloys and Compounds 2017, 710, 102-113, https://doi.org/10.1016/j.jallcom.2017.03.150.

153.Liu, K.; Qin, Y.; Muhammad, Y.; Zhu, Y.; Tang, R.; Chen, N.; Shi, H.; Zhang, H.; Tong, Z.; Yu, B. Effect of $\mathrm{Fe}_{3} \mathrm{O}_{4}$ content and microwave reaction time on the properties of $\mathrm{Fe}_{3} \mathrm{O}_{4} / \mathrm{ZnO}$ magnetic nanoparticles. $J$. Alloys and Compounds 2019, 781, 790-799, https://doi.org/10.1016/j.jallcom.2018.12.085.

154.Meng, Q.; Cui, J.; Tang, Y.; Han, Z.; Zhao, K.; Zhang, G.; Diao, Q. Solvothermal synthesis of dual-porous $\mathrm{CeO}_{2}-\mathrm{ZnO}$ composite and its enhanced acetone sensing performance. Ceramics Int. 2019, 45, 4103-4107, https://doi.org/10.1016/j.ceramint.2018.10.239.

155.Neupane, G.R.; Kaphle, A.; Hari, P. Microwave-assisted Fe-doped ZnO nanoparticles for enhancement of silicon solar cell efficiency. Solar Energy Materials and Solar Cells 2019, 201, 110073, https://doi.org/10.1016/j.solmat2019.110073. 
156.Rosowska, J.; Kaszewski, J.; Witkowski, B.; Wachnicki, Ł.; Kuryliszyn-Kudelska, I.; Godlewski, M. The effect of iron content on properties of $\mathrm{ZnO}$ nanoparticles prepared by microwave hydrothermal method. Optical Mater. 2020, 109, 110089, https://doi.org/10.1016/j.optmat.2020.110089.

157.Das, S.; Das, S.; Sutradhar, S. Effect of $\mathrm{Gd}^{3+}$ and $\mathrm{Al}^{3+}$ on optical and dielectric properties of $\mathrm{ZnO}$ nanoparticle prepared by two-step hydrothermal method. Ceramics Int. 2017, 43, 6932-6941, https://doi.org/10.1016/j.ceramint.2017.02.116.

158. Vinoditha, U.; Sarojini, B.K.; Sandeep, K.M.; Narayana, B.; Maidur, S.R.; Patil, P.S.; Balakrishna, K.M. Defects-induced nonlinear saturable absorption mechanism in europium-doped $\mathrm{ZnO}$ nanoparticles synthesized by facile hydrothermal method. Appl. Phys. A 2019, 125, 1-11, https://doi.org/10.1007/s00339019-2732-4.

159.Lin, L-Y.; Kavadiya, S.; Karakocak, B.B.; Nie, Y.; Raliya, R.; Wang, S.T.; Berezin, M.Y.; Biswas, P. $\mathrm{ZnO}_{1-\mathrm{x}} /$ carbon dots composite hollow spheres: Facile aerosol synthesis and superior $\mathrm{CO}_{2}$ photoreduction under UV, visible and near-infrared irradiation. Appl. Cataly. B: Environ. 2018, 230, 36-48, https://doi.org/10.1016/j.apcatb.2018.02.018.

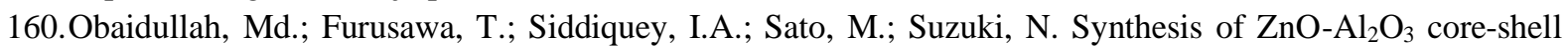
nanocomposite materials by fast and facile microwave irradiation method and investigation of their optical properties. Adv. Powder. Technol. 2017, 28, 2678-2686, https://doi.org/10.1016/j.apt.2017.07.020. 